\title{
Highly Regioselective C-H Alkylation of Alkenes Through an Aryl to Vinyl 1,4-Palladium Migration/C-C Cleavage Cascade
}

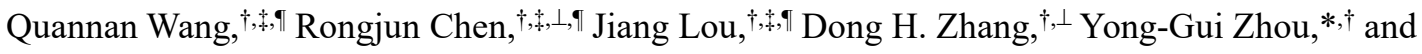

$$
\text { Zhengkun } \mathrm{Yu}^{*}, \dagger, \S
$$

'Dalian Institute of Chemical Physics, Chinese Academy of Sciences, 457 Zhongshan Road, Dalian 116023, P. R. China

University of Chinese Academy of Sciences, Beijing 100049, P. R. China

${ }^{\perp}$ State Key Laboratory of Molecular Reaction Dynamics and Center for Theoretical and

Computational Chemistry, Dalian Institute of Chemical Physics, Chinese Academy of Sciences,

Dalian 116023, P. R. China

§State Key Laboratory of Organometallic Chemistry, Shanghai Institute of Organic Chemistry, Chinese Academy of Sciences, 354 Fenglin Road, Shanghai 200032, P. R. China

TThese authors contributed equally to this work.

E-mail: zkyu@dicp.ac.cn; ygzhou@dicp.ac.cn

\section{$\underline{\text { Experimental procedures and analytical data }}$}

Contents:

1. General considerations

$\begin{array}{ll}\text { 2. Experimental procedures } & \text { S2 }\end{array}$

$\begin{array}{ll}\text { 3. X-Ray crystallographic studies } & \text { S9 }\end{array}$

$\begin{array}{lr}\text { 4. DFT studies } & \text { S10 }\end{array}$

$\begin{array}{ll}\text { 5. Analytical data } & \text { S13 }\end{array}$

$\begin{array}{ll}\text { 6. Copies of NMR spectra } & \text { S31 }\end{array}$ 


\section{General considerations}

The solvents were dried and distilled prior to use by the literature methods. ${ }^{1} \mathrm{H}$ and ${ }^{13} \mathrm{C}\left\{{ }^{1} \mathrm{H}\right\}$ NMR spectra were recorded on a Bruker DRX-400 spectrometer and all chemical shift values refer to $\delta_{\mathrm{TMS}}=0.00 \mathrm{ppm}$ or $\mathrm{CDCl}_{3}\left(\delta\left({ }^{1} \mathrm{H}\right), 7.26 \mathrm{ppm}\right.$ and $\delta\left({ }^{13} \mathrm{C}\right)$, $77.16 \mathrm{ppm})$. The HRMS analysis was obtained on a Waters GC-TOF CA156 mass spectrometer. All the chemical reagents were purchased from commercial sources and

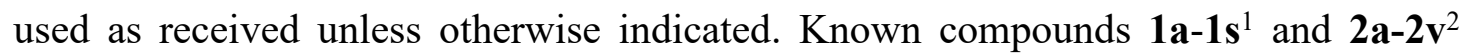
were prepared by the literature procedures, and their spectroscopic features are in good agreement with those reported in the literatures.

\section{References}

(1) Hu, T.-J.; Zhang, G.; Chen, Y.-H.; Feng, C.-G.; Lin, G.-Q. Borylation of Olefin C-H Bond via Aryl to Vinyl Palladium 1,4-Migration. J. Am. Chem. Soc. 2016, $138,2897-2900$.

(2) (a) Ren, R. G.; Wu, Z.; Xu, Y.; Zhu, C. C-C Bond-Forming Strategy by Manganese-Catalyzed Oxidative Ring-Opening Cyanation and Ethynylation of Cyclobutanol Derivatives. Angew. Chem., Int. Ed. 2016, 55, 2866-2869. (b) Dauncey, E. M.; Dighe, S. U.; Douglas, J. J.; Leonori, D. A Dual Photoredox-Nickel Strategy for Remote Functionalization via Iminyl Radicals: Radical Ring-Opening-Arylation, -Vinylation and -Alkylation Cascades. Chem. Sci. 2019, 10, 7728-7733.

\section{Experimental procedures}

\subsection{Optimization of the reaction conditions}

A mixture of 1a, cyclobutanol 2a, catalyst, ligand, additives, and base in toluene was vigorously stirred for $24 \mathrm{~h}$ under a nitrogen atmosphere. After cooling to ambient temperature, all the volatiles were evaporated under reduced pressure. The yields were determined by ${ }^{1} \mathrm{H}$ NMR spectroscopy using dimethyl terephthalate as an internal standard. 
Table S1. Optimization of the Reaction Conditions ${ }^{a}$

\begin{tabular}{|c|c|c|c|c|c|c|c|}
\hline Entry & $\begin{array}{c}\text { 1a:2a } \\
\text { (molar ratio) }\end{array}$ & Catalyst & $\begin{array}{l}\text { Ligand } \\
(\mathrm{mol} \%)\end{array}$ & $\begin{array}{l}\mathrm{Cs}_{2} \mathrm{CO}_{3} \\
\text { (equiv) }\end{array}$ & $\begin{array}{l}\text { Additive } \\
\text { (equiv) }\end{array}$ & $\begin{array}{l}\text { Yield of } \\
\mathbf{3 a}(\%)\end{array}$ & $\begin{array}{c}\mathbf{3 a}: \mathbf{4} \mathbf{a}^{b} \\
\text { (molar ratio) }\end{array}$ \\
\hline 1 & $1: 2$ & $\mathrm{PdCl}_{2}$ & $\mathrm{PCy}_{3}(10)$ & 2 & & $-/ 92^{c}$ & $<1: 20$ \\
\hline 2 & $1: 2$ & $\mathrm{PdCl}_{2}$ & $\mathrm{PCy}_{3}(10)$ & 2 & $\mathrm{H}_{2} \mathrm{O}(2)$ & $-/ 76^{c}$ & $<1: 20$ \\
\hline 3 & $1: 2$ & $\mathrm{PdCl}_{2}$ & $\mathrm{PCy}_{3}(10)$ & 2 & HFIP (2) & $-/ 15^{c}$ & $<1: 20$ \\
\hline 4 & $1: 2$ & $\mathrm{PdCl}_{2}$ & $\mathrm{PCy}_{3}(10)$ & 2 & $\mathrm{C}_{6} \mathrm{H}_{4} \mathrm{OH}(2)$ & 61 & $4.7: 1$ \\
\hline 5 & $1: 2$ & $\mathrm{PdCl}_{2}$ & $\mathrm{PCy}_{3}(10)$ & 2 & $4-\mathrm{MeC}_{6} \mathrm{H}_{4} \mathrm{OH}(2)$ & 80 & $10: 1$ \\
\hline 6 & $1: 2$ & $\mathrm{PdCl}_{2}$ & $\mathrm{PCy}_{3}(10)$ & 2 & 4- $\mathrm{FC}_{6} \mathrm{H}_{4} \mathrm{OH}(2)$ & 77 & $17.1: 1$ \\
\hline 7 & $1: 2$ & $\mathrm{PdCl}_{2}$ & $\mathrm{PCy}_{3}(10)$ & 2 & $3-\mathrm{FC}_{6} \mathrm{H}_{4} \mathrm{OH}(2)$ & 82 & $>20: 1$ \\
\hline 8 & $1: 2$ & $\mathrm{PdCl}_{2}$ & $\mathrm{PCy}_{3}(10)$ & 2 & $2-\mathrm{MeC}_{6} \mathrm{H}_{4} \mathrm{OH}(2)$ & 56 & $7: 1$ \\
\hline 9 & $1: 2$ & $\mathrm{PdCl}_{2}$ & $\mathrm{PCy}_{3}(10)$ & 2 & $2-\mathrm{ClC}_{6} \mathrm{H}_{4} \mathrm{OH}(2)$ & 85 & $>20: 1$ \\
\hline 10 & $1: 2$ & $\mathrm{PdCl}_{2}$ & $\mathrm{PCy}_{3}(10)$ & 2 & $2-\mathrm{FC}_{6} \mathrm{H}_{4} \mathrm{OH}(2)$ & 87 & $>20: 1$ \\
\hline 11 & $1: 2$ & $\mathrm{PdCl}_{2}$ & $\mathrm{PPh}_{3}(10)$ & 2 & $2-\mathrm{FC}_{6} \mathrm{H}_{4} \mathrm{OH}(2)$ & 61 & $15.2: 1$ \\
\hline 12 & $1: 2$ & $\mathrm{PdCl}_{2}$ & XPhos (10) & 2 & $2-\mathrm{FC}_{6} \mathrm{H}_{4} \mathrm{OH}(2)$ & 64 & $>20: 1$ \\
\hline 13 & $1: 2$ & $\mathrm{PdCl}_{2}$ & SPhos (10) & 2 & 2- $\mathrm{FC}_{6} \mathrm{H}_{4} \mathrm{OH}(2)$ & 61 & $>20: 1$ \\
\hline 14 & $1: 2$ & $\mathrm{PdCl}_{2}$ & $\mathrm{Ph}_{2} \mathrm{PCy}(10)$ & 2 & $2-\mathrm{FC}_{6} \mathrm{H}_{4} \mathrm{OH}(2)$ & 82 & $>20: 1$ \\
\hline 15 & $1: 2$ & $\mathrm{PdCl}_{2}$ & $\mathrm{PCy}_{3} \mathrm{BF}_{4}(10)$ & 2 & $2-\mathrm{FC}_{6} \mathrm{H}_{4} \mathrm{OH}(2)$ & 70 & $>20: 1$ \\
\hline 16 & $1: 2$ & $\mathrm{PdCl}_{2}$ & $\mathrm{PCy}_{3}(10)$ & 2.5 & 2- $\mathrm{FC}_{6} \mathrm{H}_{4} \mathrm{OH}(2)$ & 88 & $>20: 1$ \\
\hline 17 & $1: 2$ & $\mathrm{PdCl}_{2}$ & $\mathrm{PCy}_{3}(10)$ & 3 & 2- $\mathrm{FC}_{6} \mathrm{H}_{4} \mathrm{OH}(2)$ & 88 & $>20: 1$ \\
\hline 18 & $1: 2$ & $\mathrm{PdCl}_{2}$ & $\mathrm{PCy}_{3}(10)$ & 1.5 & 2- $\mathrm{FC}_{6} \mathrm{H}_{4} \mathrm{OH}(2)$ & 85 & $17: 1$ \\
\hline 19 & $1: 2$ & $\mathrm{PdCl}_{2}$ & $\mathrm{PCy}_{3}(10)$ & 2 & $2-\mathrm{FC}_{6} \mathrm{H}_{4} \mathrm{OH}(1.2)$ & 81 & $18.8: 1$ \\
\hline 20 & $1: 2$ & $\mathrm{PdCl}_{2}$ & $\mathrm{PCy}_{3}(10)$ & 2 & 2- $\mathrm{FC}_{6} \mathrm{H}_{4} \mathrm{OH}(1.6)$ & 85 & $>20: 1$ \\
\hline 21 & $1: 2$ & $\mathrm{PdCl}_{2}$ & $\mathrm{PCy}_{3}(10)$ & 2 & $2-\mathrm{FC}_{6} \mathrm{H}_{4} \mathrm{OH}(2.4)$ & 88 & $>20: 1$ \\
\hline 22 & $1: 2$ & $\mathrm{PdCl}_{2}$ & $\mathrm{PCy}_{3}(10)$ & 2 & $2-\mathrm{FC}_{6} \mathrm{H}_{4} \mathrm{OH}(2.8)$ & 87 & $>20: 1$ \\
\hline 23 & $1: 2$ & $\mathrm{PdCl}_{2}$ & $\mathrm{PCy}_{3}(5)$ & 2 & $2-\mathrm{FC}_{6} \mathrm{H}_{4} \mathrm{OH}(2)$ & 84 & $17.5: 1$ \\
\hline
\end{tabular}




\begin{tabular}{cccccccc}
\hline 24 & $1: 2$ & $\mathrm{PdCl}_{2}$ & $\mathrm{PCy}_{3}(6)$ & 2 & $2-\mathrm{FC}_{6} \mathrm{H}_{4} \mathrm{OH}(2)$ & 87 & $>20: 1$ \\
25 & $1: 2$ & $\mathrm{PdCl}_{2}$ & $\mathrm{PCy}_{3}(7)$ & 2 & $2-\mathrm{FC}_{6} \mathrm{H}_{4} \mathrm{OH}(2)$ & 87 & $>20: 1$ \\
26 & $1: 2$ & $\mathrm{PdCl}_{2}$ & $\mathrm{PCy}_{3}(8)$ & 2 & $2-\mathrm{FC}_{6} \mathrm{H}_{4} \mathrm{OH}(2)$ & 86 & $>20: 1$ \\
27 & $1: 1.5$ & $\mathrm{PdCl}_{2}$ & $\mathrm{PCy}_{3}(6)$ & 2 & $2-\mathrm{FC}_{6} \mathrm{H}_{4} \mathrm{OH}(2)$ & 88 & $>20: 1$ \\
28 & $1: 1$ & $\mathrm{PdCl}_{2}$ & $\mathrm{PCy}_{3}(6)$ & 2 & $2-\mathrm{FC}_{6} \mathrm{H}_{4} \mathrm{OH}(2)$ & 82 & $>20: 1$ \\
29 & $1: 1.5$ & $\mathrm{Pd}^{2}\left({\mathrm{OAc})_{2}}_{2}\right.$ & $\mathrm{PCy}_{3}(6)$ & 2 & $2-\mathrm{FC}_{6} \mathrm{H}_{4} \mathrm{OH}(2)$ & 61 & $>20: 1$ \\
30 & $1: 1.5$ & ${\mathrm{Pd}\left(\mathrm{MeCN}_{2} \mathrm{Cl}_{2}\right.}^{2}$ & $\mathrm{PCy}_{3}(6)$ & 2 & $2-\mathrm{FC}_{6} \mathrm{H}_{4} \mathrm{OH}(2)$ & 68 & $>20: 1$ \\
$31^{d}$ & $1: 1.5$ & $\mathrm{PdCl}_{2}$ & $\mathrm{PCy}_{3}(6)$ & 2 & $2-\mathrm{FC}_{6} \mathrm{H}_{4} \mathrm{OH}(2)$ & $87(90)^{e}$ & $>20: 1$ \\
32 & $1: 1.5$ & & $\mathrm{PCy}_{3}(6)$ & 2 & $2-\mathrm{FC}_{6} \mathrm{H}_{4} \mathrm{OH}(2)$ & 0 & \\
33 & $1: 1.5$ & $\mathrm{PdCl}_{2}$ & & 2 & $2-\mathrm{FC}_{6} \mathrm{H}_{4} \mathrm{OH}(2)$ & 0 & \\
34 & $1: 1.5$ & $\mathrm{PdCl}_{2}$ & $\mathrm{PCy}_{3}(6)$ & & $2-\mathrm{FC}_{6} \mathrm{H}_{4} \mathrm{OH}(2)$ & 0 & \\
\hline
\end{tabular}

${ }^{a}$ Conditions: 1a $(0.2 \mathrm{mmol})$, 2a, catalyst ( $5 \mathrm{~mol} \%$ ), ligand, base, additives, $2 \mathrm{~mL}$ toluene, $110{ }^{\circ} \mathrm{C}, 0.1 \mathrm{MPa} \mathrm{N}_{2}, 24$ h. ${ }^{b}$ Determined by ${ }^{1} \mathrm{H}$ NMR spectroscopy using dimethyl terephthalate as an internal standard. ${ }^{c}$ Isolated yield of $4 \mathbf{a}$. ${ }^{d} \mathbf{1 a}(0.3 \mathrm{mmol}), 3 \mathrm{~mL}$ toluene. ${ }^{e}$ Isolated yield given in parentheses.

\subsection{General procedures for the $\mathrm{C}-\mathrm{H}$ alkylation of alkenes 1}

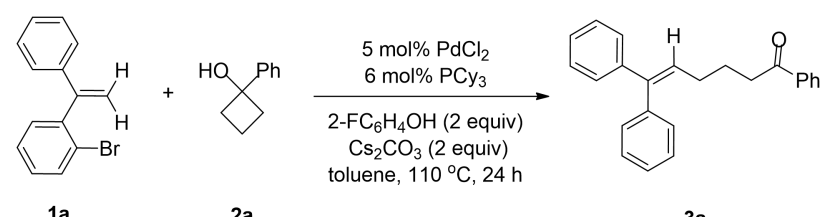

A typical procedure for the $\mathrm{C}-\mathrm{H}$ alkylation of alkenes 1 - synthesis of $3 a$ :

Under a nitrogen atmosphere a mixture of alkene $1 \mathbf{a}(77 \mathrm{mg}, 0.3 \mathrm{mmol})$, cyclobutanol 2a (73 mg, $0.45 \mathrm{mmol}), \mathrm{PdCl}_{2}(2.7 \mathrm{mg}, 0.015 \mathrm{mmol}), \mathrm{PCy}_{3}(5.0 \mathrm{mg}, 0.018 \mathrm{mmol})$, 2- $\mathrm{FC}_{6} \mathrm{H}_{4} \mathrm{OH}(67 \mathrm{mg}, 0.6 \mathrm{mmol})$, and $\mathrm{Cs}_{2} \mathrm{CO}_{3}(195 \mathrm{mg}, 0.6 \mathrm{mmol})$ in $3.0 \mathrm{~mL}$ toluene was stirred at $110{ }^{\circ} \mathrm{C}$ for $24 \mathrm{~h}$. After cooled to ambient temperature, the reaction mixture was diluted with $\mathrm{CH}_{2} \mathrm{Cl}_{2}(10 \mathrm{~mL})$, filtered through a short pad of celite, and washed with $5 \mathrm{~mL} \mathrm{CH}_{2} \mathrm{Cl}_{2}$. The combined filtrate was concentrated under reduced pressure. The resultant residue was subjected to purification by column chromatography on silica gel (eluent: petroleum ether $\left(60-90{ }^{\circ} \mathrm{C}\right) /$ EtOAc $\left.=20: 1, \mathrm{v} / \mathrm{v}\right)$ to afford 3a as a pale yellow solid (88 $\mathrm{mg}, 90 \%)$.

\subsection{General procedures for the direct alkylation of alkenes 1}




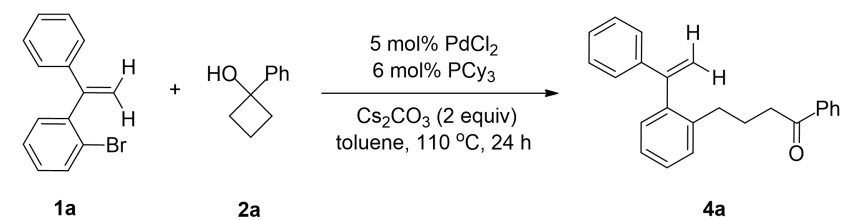

A typical procedure for the direct $\mathrm{C}-\mathrm{H}$ alkylation of alkenes 1 - synthesis of $4 \boldsymbol{a}$ : Under a nitrogen atmosphere a mixture of alkene $\mathbf{1 a}(77 \mathrm{mg}, 0.3 \mathrm{mmol})$, cyclobutanol 2a (73 mg, $0.45 \mathrm{mmol}), \mathrm{PdCl}_{2}(2.7 \mathrm{mg}, 0.015 \mathrm{mmol}), \mathrm{PCy}_{3}(5.0 \mathrm{mg}$, $0.018 \mathrm{mmol}$ ), and $\mathrm{Cs}_{2} \mathrm{CO}_{3}(195 \mathrm{mg}, 0.6 \mathrm{mmol})$ in $3.0 \mathrm{~mL}$ toluene was stirred at 110 ${ }^{\circ} \mathrm{C}$ for $24 \mathrm{~h}$. After cooled to ambient temperature, the reaction mixture was diluted with $\mathrm{CH}_{2} \mathrm{Cl}_{2}(10 \mathrm{~mL})$, filtered through a short pad of celite, and washed with $5 \mathrm{~mL}$ $\mathrm{CH}_{2} \mathrm{Cl}_{2}$. The combined filtrate was concentrated under reduced pressure. The resultant residue was subjected to purification by column chromatography on silica gel (eluent: petroleum ether $\left.\left(60-90{ }^{\circ} \mathrm{C}\right) / \mathrm{EtOAc}=20: 1, \mathrm{v} / \mathrm{v}\right)$ to afford $4 \mathbf{a}$ as a pale yellow solid $(90$ mg, 92\%).

\subsection{Deuterium-labeling experiments}

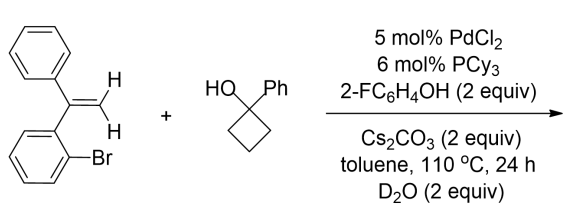

$1 \mathrm{a}$

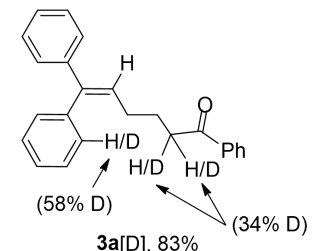

$3 a[D], 83 \%$

Under a nitrogen atmosphere a mixture of alkene 1a (77 $\mathrm{mg}, 0.3 \mathrm{mmol})$, cyclobutanol 2a (73 mg, $0.45 \mathrm{mmol}), \mathrm{PdCl}_{2}(2.7 \mathrm{mg}, 0.015 \mathrm{mmol}), \mathrm{PCy}_{3}(5.0 \mathrm{mg}$, $0.018 \mathrm{mmol}), 2-\mathrm{FC}_{6} \mathrm{H}_{4} \mathrm{OH}(67 \mathrm{mg}, 0.6 \mathrm{mmol}), \mathrm{D}_{2} \mathrm{O}(8 \mathrm{mg}, 0.4 \mathrm{mmol})$, and $\mathrm{Cs}_{2} \mathrm{CO}_{3}$ (195 mg, $0.6 \mathrm{mmol}$ ) in $3.0 \mathrm{~mL}$ toluene was stirred at $110{ }^{\circ} \mathrm{C}$ for $24 \mathrm{~h}$. After cooled to ambient temperature, the reaction mixture was diluted with $\mathrm{CH}_{2} \mathrm{Cl}_{2}(10 \mathrm{~mL})$, filtered through a short pad of celite, and washed with $5 \mathrm{~mL} \mathrm{CH} \mathrm{Cl}_{2}$. The combined filtrate was concentrated under reduced pressure. The resultant residue was subjected to purification by column chromatography on silica gel (eluent: petroleum ether (60-90 $\left.{ }^{\circ} \mathrm{C}\right) /$ EtOAc $\left.=20: 1, \mathrm{v} / \mathrm{v}\right)$ to afford $3 \mathrm{a}[\mathrm{D}]$ as a pale yellow solid $(54 \mathrm{mg}, 83 \%)$. The deuterated ratio was observed on the basis of ${ }^{1} \mathrm{H}$ NMR analysis. 
$1 \mathrm{H}$ NMR in $\mathrm{CDCl} 3(400 \mathrm{MHz})$

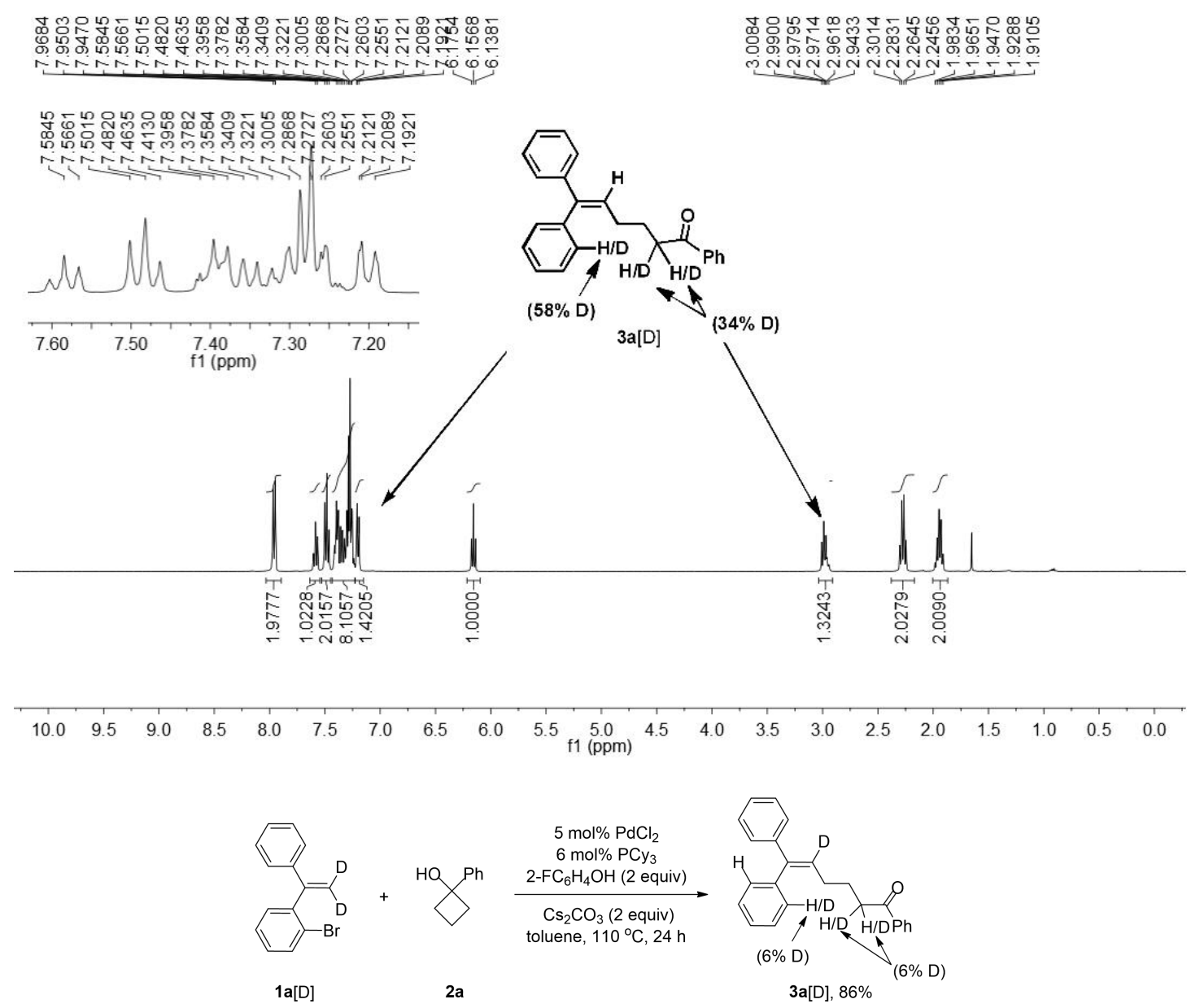

Under a nitrogen atmosphere a mixture of alkene 1a[D] (77 $\mathrm{mg}, 0.3 \mathrm{mmol}$ ), cyclobutanol 2a (73 mg, $0.45 \mathrm{mmol}), \mathrm{PdCl}_{2}(2.7 \mathrm{mg}, 0.015 \mathrm{mmol}), \mathrm{PCy}_{3}(5.0 \mathrm{mg}$, $0.018 \mathrm{mmol}), 2-\mathrm{FC}_{6} \mathrm{H}_{4} \mathrm{OH}(67 \mathrm{mg}, 0.6 \mathrm{mmol})$, and $\mathrm{Cs}_{2} \mathrm{CO}_{3}(195 \mathrm{mg}, 0.6 \mathrm{mmol})$ in 3.0 $\mathrm{mL}$ toluene was stirred at $110{ }^{\circ} \mathrm{C}$ for $24 \mathrm{~h}$. After cooled to ambient temperature, the reaction mixture was diluted with $\mathrm{CH}_{2} \mathrm{Cl}_{2}(10 \mathrm{~mL})$, filtered through a short pad of celite, and washed with $5 \mathrm{~mL} \mathrm{CH} \mathrm{Cl}_{2}$. The combined filtrate was concentrated under reduced pressure. The resultant mixture was subjected to purification by column chromatography on silica gel (eluent: petroleum ether $\left.\left(60-90{ }^{\circ} \mathrm{C}\right) / \mathrm{EtOAc}=20: 1, \mathrm{v} / \mathrm{v}\right)$ to afford $3 \mathrm{a}[\mathrm{D}]$ as a pale yellow solid (56 $\mathrm{mg}, 86 \%$ ). The deuterated ratio was observed on the basis of ${ }^{1} \mathrm{H}$ NMR analysis. 
WQN-1758W

$1 \mathrm{H}$ NMR in $\mathrm{CDCl} 3(400 \mathrm{MHz})$
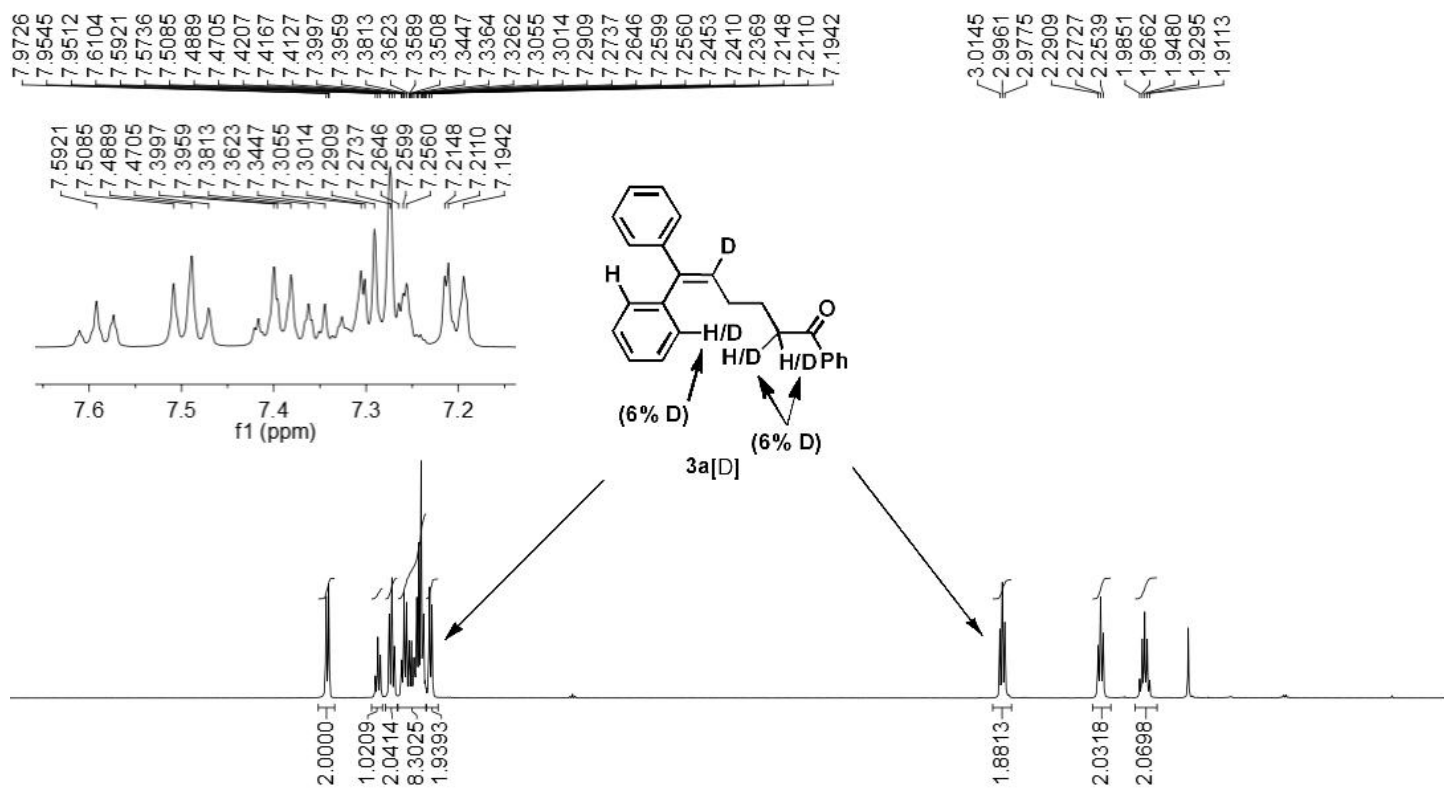

\subsection{Kinetic isotope effect (KIE) experiments}

The $\mathrm{C} H(\mathrm{D})$ alkylation reactions of $1 \mathbf{a}$ and its deuterated form, 1a[D], were carried out in a parallel manner under the optimized conditions. The NMR yields from the reactions were carefully checked by the signal integration of the target product 3a with dimethyl terephthalate as the internal standard. The $\mathrm{k}_{\mathrm{H}} / \mathrm{k}_{\mathrm{D}}$ value was calculated according to the yields of $\mathbf{3 a}$ and $\mathbf{3 a}[\mathrm{D}]$ from the reactions at time intervals of $1 \mathrm{~h}, 2 \mathrm{~h}$, and $3 \mathrm{~h}$.

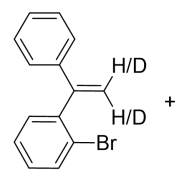

1a, 1a[D]

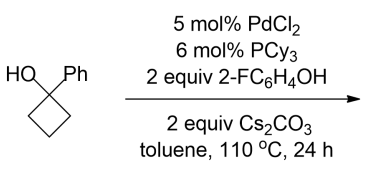

2 equiv $\mathrm{Cs}_{2} \mathrm{CO}_{3}$
toluene, $110{ }^{\circ} \mathrm{C}, 24 \mathrm{~h}$

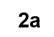

time (h)

3a $3 a[D]$

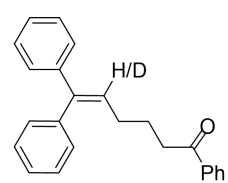

$3 a, 3 a[D]$

\subsection{Gram-scale preparation of compound 5a}

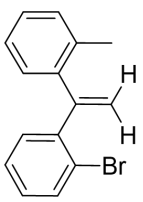

1d

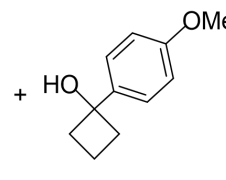

2b

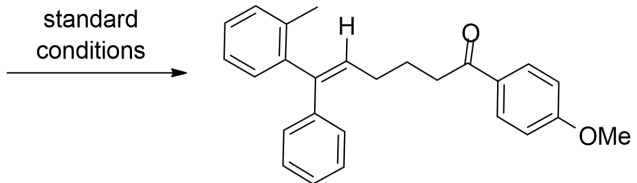

$5 a$

Under a nitrogen atmosphere a mixture of alkene 1d (980 mg, $3.6 \mathrm{mmol}$ ), 
cyclobutanol $2 \mathbf{b}$ (962.0 mg, $5.4 \mathrm{mmol}), \mathrm{PdCl}_{2}(32 \mathrm{mg}, 0.18 \mathrm{mmol}), \mathrm{PCy}_{3}(61.0 \mathrm{mg}$, $0.216 \mathrm{mmol}), 2-\mathrm{FC}_{6} \mathrm{H}_{4} \mathrm{OH}(807.0 \mathrm{mg}, 7.2 \mathrm{mmol})$, and $\mathrm{Cs}_{2} \mathrm{CO}_{3}(2.4 \mathrm{~g}, 7.2 \mathrm{mmol})$ in 36 $\mathrm{mL}$ toluene was stirred at $110{ }^{\circ} \mathrm{C}$ for $24 \mathrm{~h}$. After cooled to ambient temperature, the reaction mixture was diluted with $\mathrm{CH}_{2} \mathrm{Cl}_{2}(30 \mathrm{~mL})$, filtered through a short pad of celite, and washed with $30 \mathrm{~mL} \mathrm{CH}_{2} \mathrm{Cl}_{2}$. The combined filtrate was concentrated under reduced pressure. The resultant residue was subjected to purification by column chromatography on silica gel (eluent: petroleum ether $\left.\left(60-90{ }^{\circ} \mathrm{C}\right) / \operatorname{EtOAc}=10: 1, \mathrm{v} / \mathrm{v}\right)$ to afford $\mathbf{5 a}$ as a pale yellow solid (1.2 $\mathrm{g}, 90 \%)$.

\subsection{Procedure for the preparation of reduced product 9}
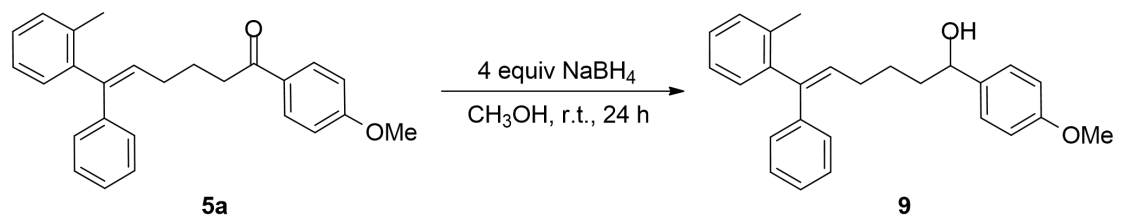

To a stirred solution of $5 \mathbf{a}(111.0 \mathrm{mg}, 0.3 \mathrm{mmol})$ in $\mathrm{MeOH}(2 \mathrm{~mL})$ was added $\mathrm{NaBH}_{4}(45.0 \mathrm{mg}, 1.2 \mathrm{mmol})$ at ambient temperature. After stirring for $24 \mathrm{~h}$ at this temperature, all the volatiles in the mixture were evaporated under reduced pressure. The resultant residue was purified by silica gel column chromatography (eluent: petroleum ether $\left(60-90{ }^{\circ} \mathrm{C}\right) /$ ethyl acetate $\left.=10: 1, \mathrm{v} / \mathrm{v}\right)$ to afford the pure product 9 (105 mg, 94\%).

\subsection{Procedure for the preparation of 1,6-diene 10}
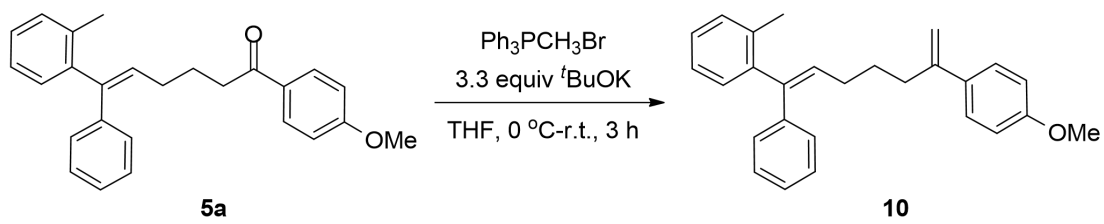

To a stirred solution of methyltriphenylphosphonium bromide $(214.0 \mathrm{mg}, 0.6$ $\mathrm{mmol})$ in THF $(2 \mathrm{~mL})$ was added potassium tert-butoxide $(111.1 \mathrm{mg}, 0.99 \mathrm{mmol})$ at 0 ${ }^{\circ} \mathrm{C}$ under a nitrogen atmosphere. The reaction mixture was stirred at $0{ }^{\circ} \mathrm{C}$ for $20 \mathrm{~min}$, and then a solution of $\mathbf{5 a}(111.0 \mathrm{mg}, 0.3 \mathrm{mmol})$ in THF $(1 \mathrm{~mL})$ was added dropwise for $10 \mathrm{~min}$. The resulting mixture was warmed gradually to ambient temperature and kept stirring for $3 \mathrm{~h}$. The resultant reaction solution was filtered over Celite, and washed with $\mathrm{CH}_{2} \mathrm{Cl}_{2}(2 \times 10 \mathrm{~mL})$. The combined filtrate was concentrated under reduced pressure to yield a residue which was further purified over silica gel flash 
column chromatography (eluent: petroleum ether $\left(60-90^{\circ} \mathrm{C}\right) /$ ethyl acetate $\left.=20: 1, \mathrm{v} / \mathrm{v}\right)$ to afford the pure product $\mathbf{1 0}(103.0 \mathrm{mg}, 94 \%)$.

\section{X-Ray crystallographic studies}

Single crystal X-ray diffraction studies for compound $\mathbf{3 b}$ were carried out on a SMART APEX diffractometer with graphite-monochromated Mo $\mathrm{K} \alpha$ radiation $(\lambda=$ $0.71073 \AA$ ). Cell parameters were obtained by global refinement of the positions of all collected reflections. Intensities were corrected for Lorentz and polarization effects and empirical absorption. The structure was solved by direct methods and refined by full-matrix least squares on $\boldsymbol{F}^{2}$. All non-hydrogen atoms were refined anisotropically. All hydrogen atoms were placed in calculated positions. Structure solution and refinement were performed by using the SHELXL-97 package. The X-ray crystallographic files, in CIF format, are available from the Cambridge Crystallographic Data Centre on quoting the deposition number CCDC 1891215 for 3b. Copies of this information may be obtained free of charge from The Director, CCDC, 12 Union Road, Cambridge CB2 IEZ, UK (Fax: +44-1223-336033; e-mail: deposit@ccdc.cam.ac.uk or www: http://www.ccdc.cam.ac.uk).

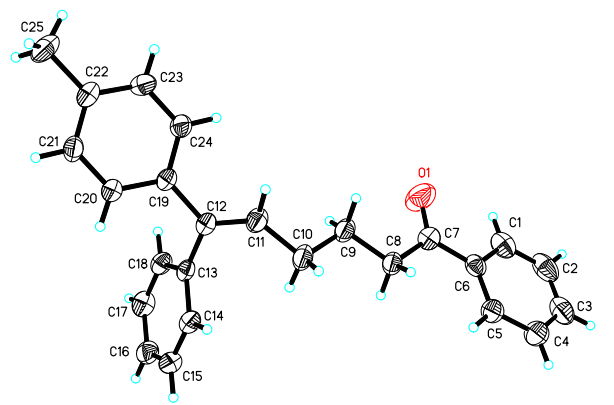

Figure S1. Molecular structure of $\mathbf{3 b}$.

Table S2. Crystal Data and Structure Refinement for 3b

\begin{tabular}{ll}
\hline Identification code & WQN-1601W \\
Empirical formula & C25 H24 O \\
Formula weight & 340.44 \\
Temperature & $293(2) \mathrm{K}$ \\
Wavelength & $0.71073 \AA$ \\
Crystal system & Triclinic \\
Space group & $\mathrm{P}-1$
\end{tabular}




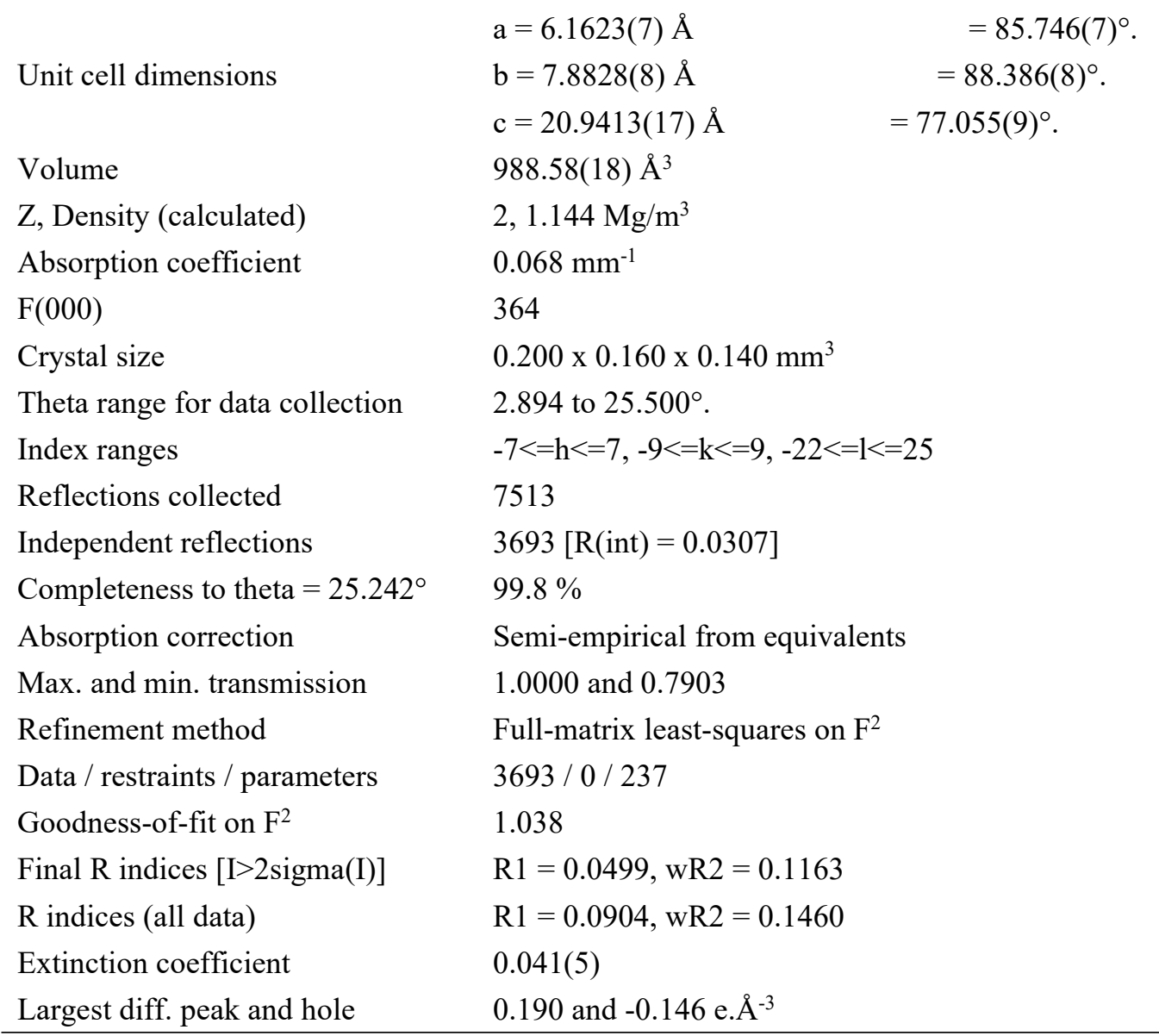

\section{DFT studies}

\subsection{Complete reference for Gaussian 16}

Gaussian 16, Revision A.03. M. J. Frisch, G. W. Trucks, H. B. Schlegel, G. E. Scuseria,M. A. Robb, J. R. Cheeseman, G. Scalmani, V. Barone, G. A. Petersson, H. Nakatsuji, X. Li, M. Caricato, A. V. Marenich, J. Bloino, B. G. Janesko, R. Gomperts, B. Mennucci, H. P. Hratchian, J. V. Ortiz, A. F. Izmaylov, J. L. Sonnenberg, D. Williams-Young, F. Ding, F. Lipparini, F. Egidi, J. Goings, B. Peng, A. Petrone, T. Henderson, D. Ranasinghe, V. G. Zakrzewski, J. Gao, N. Rega, G. Zheng, W. Liang, M. Hada, M. Ehara, K. Toyota, R. Fukuda, J. Hasegawa, M. Ishida, T. Nakajima, Y. Honda, O. Kitao, H. Nakai, T. Vreven, K. Throssell, J. A. Montgomery, Jr., J. E. Peralta, F. Ogliaro, M. J. Bearpark, J. J. Heyd, E. N. Brothers, K. N. Kudin, V. N. Staroverov, T. A. Keith, R. Kobayashi, J. Normand, K. Raghavachari, A. P. Rendell, J. C. Burant, S. S. Iyengar, J. Tomasi, M. Cossi, J. M. Millam, M. Klene, C. Adamo, R. Cammi, J. W. Ochterski, R. L. Martin, K. Morokuma, O. Farkas, J. B. Foresman, and 
D. J. Fox, Gaussian, Inc., Wallingford CT, 2016.

4.2 B3LYP absolute calculation energies (Hartree), energy corrections (Hartree), and imaginary frequency $\left(\mathrm{cm}^{-1}\right)$

\begin{tabular}{|c|c|c|c|c|}
\hline Geometry & $\begin{array}{c}\text { E(B3LYP/ } \\
6-311++G(2 d f, 2 p)-S D D)\end{array}$ & $\mathbf{G}(\text { corr-solv })^{[\mathbf{a}]}$ & $\mathbf{G}(\text { corr-ther })^{[\mathbf{b}]}$ & $I^{[c]}$ \\
\hline Int0 & -4290.0659498 & -0.03193811 & 0.591768 & - \\
\hline TSI1 & -5037.94950734 & -0.08720842 & 0.773631 & -75.92 \\
\hline IntI1 & -2178.95757442 & -0.03497813 & 0.764298 & - \\
\hline TSI2 & -2178.93656306 & -0.03495251 & 0.767923 & -353.89 \\
\hline IntI2 & -2178.97960207 & -0.0363833 & 0.760909 & - \\
\hline TSIII & -4574.23930256 & -0.07629373 & 0.590198 & -817.13 \\
\hline IntII1 & -2122.12496863 & -0.03935423 & 0.666144 & - \\
\hline TSII2 & -2122.0963835 & -0.03762145 & 0.661479 & -1392.97 \\
\hline IntIII2 & -2585.8688654 & -0.04333315 & 0.846433 & - \\
\hline TSIII3 & -2585.8565975 & -0.04108307 & 0.844805 & -705.18 \\
\hline IntII3 & -2178.95519598 & -0.03872662 & 0.758525 & - \\
\hline TSII4 & -2178.93587093 & -0.03853559 & 0.762131 & -370.80 \\
\hline IntII4 & -2178.97960635 & -0.03907627 & 0.75948 & - \\
\hline TSII5 & -2178.90015057 & -0.03976506 & 0.756851 & -1559.07 \\
\hline IntII5 & -2178.9385659 & -0.04213305 & 0.7613 & - \\
\hline $\mathrm{CsCO}_{3}^{-}$ & -284.109138778 & -0.081815352 & -0.028942 & - \\
\hline $\mathrm{HCO}_{3}^{-}$ & -264.567448972 & -0.054939337 & -0.008732 & - \\
\hline $\mathrm{CsBr}$ & -2594.38576148 & -0.02918753 & -0.035241 & - \\
\hline 2-fluorophenol & -406.872543124 & -0.008228704 & 0.055194 & - \\
\hline 1-phenylcyclobutanol & -463.679834264 & -0.012771474 & 0.146905 & - \\
\hline
\end{tabular}

${ }^{[a]}$ The solvation energy corrections calculated at the M052x/6-31G(d)-SDD level with the SMD solvation model for toluene minus electronic energy in gas phase. ${ }^{[b]}$ The thermal correction to Gibbs free energy calculated by B3LYP/6-31+G(d)-SDD at $383.15 \mathrm{~K}$ in gas phase. ${ }^{[\mathrm{c}]}$ The B3LYP/6-31+G(d)-SDD calculated imaginary frequencies for the transition states in gas phase.

4.3 Calculated relative free energies $(\mathrm{kcal} / \mathrm{mol})$ of relevant transition states and intermediates for the reaction of 3, 4 and 5-membered cycloalkanols at the B3LYP/6-311++G(2df,2p)//SMD (toluene)-M052x/6-31G(d)-SDD, and molecular geometries were optimized at the B3LYP/3-21G-SDD level for simplification

\begin{tabular}{cccccc}
\hline & Ring-opening & Ring-opening & TS in & Direct & 1,4-palladium \\
Cycloal- & TS in direct & TS in migration & migration & inter- & migration \\
kanols & process & process & process & mediate & intermediate \\
& IntI1 $\rightarrow$ TSI2 & IntII3 $\rightarrow$ TSII4 & IntII1 $\rightarrow$ TSII2 & IntI2 & IntII4 \\
& & & & \\
\hline
\end{tabular}




\begin{tabular}{lccccc}
\hline 3membered & 3.337 & 4.260 & 15.834 & -41.988 & -41.933 \\
4membered & 12.287 & 10.214 & 15.834 & -35.226 & -42.130 \\
& & & & & \\
5 membered & 21.534 & 20.978 & 15.834 & -19.705 & -24.473 \\
\hline
\end{tabular}

As can be seen, the energy needed for ring-opening process increase significantly along with the size of cycloalkanols. We initially consider the reaction of 3-membered cycloalkanols, the migration process is the rate-limiting step obviously, and the free energy of IntI2 is approximately the same as the migration intermediate IntII4. Therefore, Pathway A is kinetically favored, leading to the formation of the direct alkylation product. Further for 4-membered substrate, the free energy barrier of the two pathways are close. However, it is noteworthy that intermediate IntII4 is thermally more stable, hence we found the rare aryl to vinyl 1,4-Pd migration reaction. Finally, as revealed in table, the reaction is not likely to happen for the more stable 5-membered cycloalkanols in terms of the high energy barrier toward ring opening, which is consistent with previous Scheme 2. Notably, the absolute calculation energies are summarized in 4.4 .

4.4 B3LYP absolute calculation energies (Hartree), energy corrections (Hartree), and imaginary frequency $\left(\mathrm{cm}^{-1}\right)$

\begin{tabular}{|c|c|c|c|c|}
\hline Geometry & $\begin{array}{c}\text { E(B3LYP/ } \\
6-311++G(2 d f, 2 p) \\
- \text { SDD })\end{array}$ & $\mathbf{G}(\text { corr-solv })^{[\mathbf{a}]}$ & $\mathbf{G}(\text { corr-ther })^{[b]}$ & $I^{[c]}$ \\
\hline Int0 & -4290.06033701 & -0.03136397 & 0.599679 & - \\
\hline IntIII & -2122.11579083 & -0.03914737 & 0.677222 & - \\
\hline TSII2 & -2122.08737507 & -0.03655985 & 0.671451 & -1060.1 \\
\hline 2-fluorophenol & -406.870946879 & -0.008207937 & 0.056545 & - \\
\hline $\mathrm{CsCO}_{3}^{-}$ & -284.107578334 & -0.082345915 & -0.030261 & - \\
\hline $\mathrm{HCO}_{3}^{-}$ & -264.564264219 & -0.054397638 & -0.009827 & - \\
\hline $\mathrm{CsBr}$ & -2594.38578389 & -0.02898215 & -0.035143 & - \\
\hline IntI1-3membered & -2178.94352457 & -0.03354797 & 0.774901 & - \\
\hline TSI2-3membered & -2178.93915108 & -0.0344809 & 0.776778 & -140.79 \\
\hline IntI2-3membered & -2178.97552905 & -0.03569956 & 0.772384 & - \\
\hline IntII3-3membered & -2178.94359997 & -0.03718925 & 0.770588 & - \\
\hline TSII4-3membered & -2178.93523102 & -0.03864775 & 0.770466 & -89.95 \\
\hline
\end{tabular}




\begin{tabular}{ccccc} 
IntII4-3membered & -2178.97200509 & -0.03751006 & 0.770757 & - \\
1-benzylcyclopropanol & -463.671454177 & -0.013882389 & 0.147212 & - \\
IntI1-4membered & -2178.94588036 & -0.03366362 & 0.775335 & - \\
TSI2-4membered & -2178.92781395 & -0.03386632 & 0.777051 & -329.18 \\
IntI2-4membered & -2178.96860154 & -0.034929 & 0.772307 & - \\
IntII3-4membered & -2178.94315694 & -0.03725457 & 0.769237 & - \\
TSII4-4membered & -2178.92796813 & -0.03789601 & 0.770966 & -346.08 \\
IntII4-4membered & -2178.97265589 & -0.03845746 & 0.768888 & - \\
1-phenylcyclobutanol & -463.676878525 & -0.012811927 & 0.148412 & - \\
IntI1-5membered & -2218.30789371 & -0.03288365 & 0.804563 & - \\
TSI2-5membered & -2218.27182661 & -0.03395904 & 0.803888 & -343.29 \\
IntI2-5membered & -2218.3025075 & -0.03656944 & 0.799659 & - \\
IntII3-5membered & -2218.30487113 & -0.0376344 & 0.796504 & - \\
TSII4-5membered & -2218.27220155 & -0.03817657 & 0.797807 & -358.39 \\
IntII4-5membered & -2218.30470703 & -0.03909939 & 0.79679 & - \\
1-Phenylcyclopentanol & -503.038133947 & -0.01340907 & 0.177336 & - \\
\hline
\end{tabular}

${ }^{[a]}$ The solvation energy corrections calculated at the M052x/6-31G(d)-SDD level with the SMD solvation model for toluene minus electronic energy in gas phase. ${ }^{[b]}$ The thermal correction to Gibbs free energy calculated by B3LYP/3-21G-SDD at $383.15 \mathrm{~K}$ in gas phase. [c]The B3LYP/3-21G-SDD calculated imaginary frequencies for the transition states in gas phase.

\section{Analytical data}

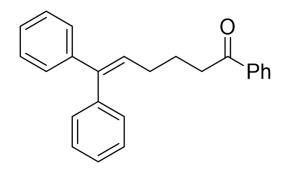

1,6,6-Triphenylhex-5-en-1-one (3a): $88 \mathrm{mg}, 90 \%$ yield, pale yellow solid, m.p.: 60-62 ${ }^{\circ} \mathrm{C} .{ }^{1} \mathrm{H}$ NMR $\left(400 \mathrm{MHz}, \mathrm{CDCl}_{3}\right) \delta 7.96(\mathrm{~m}, 2 \mathrm{H}$, aromatic $\mathrm{CH}), 7.58(\mathrm{~m}, 1 \mathrm{H}$, aromatic $\mathrm{CH}), 7.48(\mathrm{dd}, J=10.5$ and $4.7 \mathrm{~Hz}, 2 \mathrm{H}$, aromatic $\mathrm{CH}), 7.31(\mathrm{~m}, 10 \mathrm{H}$, aromatic $\mathrm{CH}), 6.17(\mathrm{t}, J=7.5 \mathrm{~Hz}, 1 \mathrm{H}, \mathrm{C}=\mathrm{CH}), 2.99\left(\mathrm{t}, J=7.4 \mathrm{~Hz}, 2 \mathrm{H}, \mathrm{PhCOCH}_{2}\right)$, 2.29 (q, $\left.J=7.4 \mathrm{~Hz}, 2 \mathrm{H}, \mathrm{C}=\mathrm{CHCH}_{2}\right), 1.96\left(\mathrm{p}, J=7.5 \mathrm{~Hz}, 2 \mathrm{H}, \mathrm{C}=\mathrm{CHCH}_{2} \mathrm{CH}_{2}\right.$ ). ${ }^{13} \mathrm{C}\left\{{ }^{1} \mathrm{H}\right\}$ NMR $\left(100 \mathrm{MHz}, \mathrm{CDCl}_{3}\right) \delta 200.1(\mathrm{Cq}, \mathrm{C}=\mathrm{O}), 142.65,142.50,140.1$, and $137.1(\mathrm{Cq}), 133.0,130.0,129.1,128.6,128.3,128.2,128.1,127.3,127.0$, and 127.0 (aromatic $\mathrm{CH}), 38.1\left(\mathrm{PhCOCH}_{2}\right), 29.4\left(\mathrm{C}=\mathrm{CHCH}_{2}\right), 24.5\left(\mathrm{C}=\mathrm{CHCH}_{2} \mathrm{CH}_{2}\right) . \mathrm{HRMS}$ Calcd for $\mathrm{C}_{24} \mathrm{H}_{22} \mathrm{O}[\mathrm{M}+\mathrm{H}]^{+}: 327.1749$, found: 327.1748 .

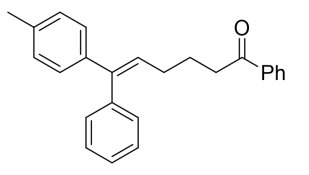

(E)-1,6-Diphenyl-6-(p-tolyl)hex-5-en-1-one (3b): 93 mg, 91\% yield, white solid, m.p.: $48-50{ }^{\circ} \mathrm{C} .{ }^{1} \mathrm{H}$ NMR $\left(400 \mathrm{MHz}, \mathrm{CDCl}_{3}\right) \delta 7.94(\mathrm{~m}, 2 \mathrm{H}$, aromatic $\mathrm{CH}), 7.56(\mathrm{t}, J$ 
$=7.4 \mathrm{~Hz}, 1 \mathrm{H}$, aromatic $\mathrm{CH}), 7.46(\mathrm{t}, J=7.6 \mathrm{~Hz}, 2 \mathrm{H}$, aromatic $\mathrm{CH}), 7.35(\mathrm{~m}, 3 \mathrm{H}$, aromatic $\mathrm{CH}), 7.15(\mathrm{~m}, 6 \mathrm{H}$, aromatic $\mathrm{CH}), 6.11(\mathrm{t}, J=7.5 \mathrm{~Hz}, 1 \mathrm{H}, \mathrm{C}=\mathrm{CH}), 2.97(\mathrm{t}, J$ $\left.=7.4 \mathrm{~Hz}, 2 \mathrm{H}, \mathrm{PhCOCH}_{2}\right), 2.35\left(\mathrm{~s}, 3 \mathrm{H}, \mathrm{CH}_{3}\right), 2.25\left(\mathrm{q}, J=7.4 \mathrm{~Hz}, 2 \mathrm{H}, \mathrm{C}=\mathrm{CHCH}_{2}\right)$, $1.93\left(\mathrm{~m}, 2 \mathrm{H}, \mathrm{C}=\mathrm{CHCH}_{2} \mathrm{CH}_{2}\right) .{ }^{13} \mathrm{C}\left\{{ }^{1} \mathrm{H}\right\} \mathrm{NMR}\left(100 \mathrm{MHz}, \mathrm{CDCl}_{3}\right) \delta 200.2(\mathrm{Cq}, \mathrm{C}=\mathrm{O})$, $142.3,140.3,139.9,137.1$, and $136.8(\mathrm{Cq}), 133.0,130.0,128.9,128.6,128.3,128.2$, 128.1, 127.2, and 127.0 (aromatic $\mathrm{CH}), 38.1\left(\mathrm{PhCOCH}_{2}\right), 29.4\left(\mathrm{C}=\mathrm{CHCH}_{2}\right), 24.6$ $\left(\mathrm{C}=\mathrm{CHCH}_{2} \mathrm{CH}_{2}\right), 21.1\left(\mathrm{CH}_{3}\right)$. HRMS Calcd for $\mathrm{C}_{25} \mathrm{H}_{24} \mathrm{O}[\mathrm{M}+\mathrm{H}]^{+}:$341.1905, found: 341.1901 .

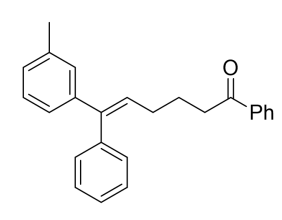

(E)-1,6-Diphenyl-6-(m-tolyl)hex-5-en-1-one (3c): $93 \mathrm{mg}$, 91\% yield, white solid, m.p.: 84-86 ${ }^{\circ} \mathrm{C} .{ }^{1} \mathrm{H}$ NMR $\left(400 \mathrm{MHz}, \mathrm{CDCl}_{3}\right) \delta 7.94(\mathrm{~d}, J=7.4 \mathrm{~Hz}, 2 \mathrm{H}$, aromatic $\mathrm{CH}), 7.56(\mathrm{t}, J=7.3 \mathrm{~Hz}, 1 \mathrm{H}$, aromatic $\mathrm{CH}), 7.46(\mathrm{t}, J=7.6 \mathrm{~Hz}, 2 \mathrm{H}$, aromatic $\mathrm{CH}), 7.34(\mathrm{~m}, 3 \mathrm{H}$, aromatic $\mathrm{CH}), 7.17(\mathrm{t}, J=7.3 \mathrm{~Hz}, 3 \mathrm{H}$, aromatic $\mathrm{CH})$, $7.05(\mathrm{dd}, J=13.0$ and $8.8 \mathrm{~Hz}, 3 \mathrm{H}$, aromatic $\mathrm{CH}), 6.12(\mathrm{t}, J=7.5 \mathrm{~Hz}, 1 \mathrm{H}, \mathrm{C}=\mathrm{CH})$, 2.97 (t, $\left.J=7.4 \mathrm{~Hz}, 2 \mathrm{H}, \mathrm{PhCOCH}_{2}\right), 2.32\left(\mathrm{~s}, 3 \mathrm{H}, \mathrm{CH}_{3}\right), 2.24(\mathrm{dd}, J=14.8$ and $7.4 \mathrm{~Hz}$, $\left.2 \mathrm{H}, \mathrm{C}=\mathrm{CHCH}_{2}\right), 1.92\left(\mathrm{~m}, 2 \mathrm{H}, \mathrm{C}=\mathrm{CHCH}_{2} \mathrm{CH}_{2}\right) .{ }^{13} \mathrm{C}\left\{{ }^{1} \mathrm{H}\right\} \mathrm{NMR}\left(100 \mathrm{MHz}, \mathrm{CDCl}_{3}\right) \delta$ $200.2(\mathrm{Cq}, \mathrm{C}=\mathrm{O}), 142.7,142.6,140.3,137.7$, and 137.1 (Cq), 133.0, 130.0, 129.0, 128.6, 128.3, 128.1, 128.1, 128.0, 127.8, 127.0, and 124.6 (aromatic CH), 38.1 $\left(\mathrm{PhCOCH}_{2}\right), 29.4\left(\mathrm{C}=\mathrm{CHCH}_{2}\right), 24.6\left(\mathrm{C}=\mathrm{CHCH}_{2} \mathrm{CH}_{2}\right), 21.6\left(\mathrm{CH}_{3}\right)$. HRMS Calcd for $\mathrm{C}_{25} \mathrm{H}_{24} \mathrm{O}[\mathrm{M}+\mathrm{H}]^{+}:$341.1905, found: 341.1900 .

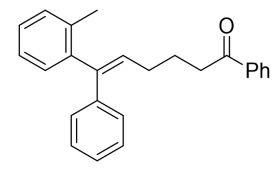

(E)-1,6-Diphenyl-6-(o-tolyl)hex-5-en-1-one (3d): $96 \mathrm{mg}$, 94\% yield, pale yellow solid, m.p.: 94-95 ${ }^{\circ} \mathrm{C} .{ }^{1} \mathrm{H}$ NMR $\left(400 \mathrm{MHz}, \mathrm{CDCl}_{3}\right) \delta 8.00(\mathrm{~m}, 2 \mathrm{H}$, aromatic $\mathrm{CH}), 7.60(\mathrm{~m}, 1 \mathrm{H}$, aromatic $\mathrm{CH}), 7.50(\mathrm{~m}, 2 \mathrm{H}$, aromatic $\mathrm{CH}), 7.35(\mathrm{~m}, 2 \mathrm{H}$, aromatic $\mathrm{CH}), 7.26(\mathrm{~m}, 6 \mathrm{H}$, aromatic $\mathrm{CH}), 7.18(\mathrm{~m}, 1 \mathrm{H}$, aromatic $\mathrm{CH}), 5.79(\mathrm{t}, J=7.4 \mathrm{~Hz}, 1 \mathrm{H}$, $\mathrm{C}=\mathrm{C} H), 3.06\left(\mathrm{~m}, 2 \mathrm{H}, \mathrm{PhCOCH}_{2}\right), 2.51\left(\mathrm{dd}, J=14.9\right.$ and $\left.7.4 \mathrm{~Hz}, 2 \mathrm{H}, \mathrm{C}=\mathrm{CHCH}_{2}\right)$, $2.12\left(\mathrm{~s}, 3 \mathrm{H}, \mathrm{CH}_{3}\right), 2.01\left(\mathrm{~m}, 2 \mathrm{H}, \mathrm{C}=\mathrm{CHCH}_{2} \mathrm{CH}_{2}\right) .{ }^{13} \mathrm{C}\left\{{ }^{1} \mathrm{H}\right\} \mathrm{NMR}\left(100 \mathrm{MHz}, \mathrm{CDCl}_{3}\right)$ 
$\delta 200.1(\mathrm{Cq}, \mathrm{C}=\mathrm{O}), 143.9,142.5,140.2,137.1$, and 136.1 (Cq), 133.0, 131.3, 130.3, 130.2, 129.4, 128.6, 128.1, 128.0, 127.2, 126.8, and 125.6 (aromatic $\mathrm{CH}$ ), 38.1 $\left(\mathrm{PhCOCH}_{2}\right), 28.9\left(\mathrm{C}=\mathrm{CHCH}_{2}\right), 24.7\left(\mathrm{C}=\mathrm{CHCH}_{2} \mathrm{CH}_{2}\right), 20.6\left(\mathrm{CH}_{3}\right)$. HRMS Calcd for $\mathrm{C}_{25} \mathrm{H}_{24} \mathrm{O}[\mathrm{M}+\mathrm{H}]^{+}: 341.1905$, found: 341.1908 .

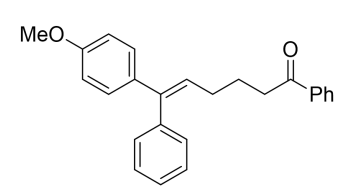

(E)-6-(4-Methoxyphenyl)-1,6-diphenylhex-5-en-1-one (3e): 95 mg, 89\% yield, pale yellow solid, m.p.: 74-75 ${ }^{\circ} \mathrm{C} .{ }^{1} \mathrm{H}$ NMR (400 MHz, $\left.\mathrm{CDCl}_{3}\right) \delta 7.94$ (d, J=7.4 Hz, $2 \mathrm{H}$, aromatic $\mathrm{CH}), 7.56(\mathrm{t}, J=7.3 \mathrm{~Hz}, 1 \mathrm{H}$, aromatic $\mathrm{CH}), 7.46(\mathrm{t}, J=7.6 \mathrm{~Hz}, 2 \mathrm{H}$, aromatic $\mathrm{CH}), 7.33(\mathrm{~m}, 3 \mathrm{H}$, aromatic $\mathrm{CH}), 7.19(\mathrm{~m}, 4 \mathrm{H}$, aromatic $\mathrm{CH}), 6.82(\mathrm{~d}, J=$ $8.8 \mathrm{~Hz}, 2 \mathrm{H}$, aromatic $\mathrm{CH}), 6.05(\mathrm{t}, J=7.5 \mathrm{~Hz}, 1 \mathrm{H}, \mathrm{C}=\mathrm{CH}), 3.80\left(\mathrm{~s}, 3 \mathrm{H}, \mathrm{OCH}_{3}\right), 2.96$ ( $\left.\mathrm{t}, J=7.4 \mathrm{~Hz}, 2 \mathrm{H}, \mathrm{PhCOCH}_{2}\right), 2.23$ (q, $\left.J=7.4 \mathrm{~Hz}, 2 \mathrm{H}, \mathrm{C}=\mathrm{CHCH}_{2}\right), 1.91(\mathrm{~m}, 2 \mathrm{H}$, $\left.\mathrm{C}=\mathrm{CHCH}_{2} \mathrm{CH}_{2}\right) .{ }^{13} \mathrm{C}\left\{{ }^{1} \mathrm{H}\right\} \mathrm{NMR}\left(100 \mathrm{MHz}, \mathrm{CDCl}_{3}\right) \delta 200.2(\mathrm{Cq}, \mathrm{C}=\mathrm{O}), 158.8,141.9$, 140.4, 137.1, and 135.4 (Cq), 133.0, 129.9, 128.6, 128.4, 128.3, 128.1, 127.3, 127.0, and 113.6 (aromatic $\mathrm{CH}), 55.3\left(\mathrm{OCH}_{3}\right), 38.1\left(\mathrm{PhCOCH}_{2}\right), 29.4\left(\mathrm{C}=\mathrm{CHCH}_{2}\right), 24.6$ $\left(\mathrm{C}=\mathrm{CHCH}_{2} \mathrm{CH}_{2}\right)$. HRMS Calcd for $\mathrm{C}_{25} \mathrm{H}_{24} \mathrm{O}_{2}[\mathrm{M}+\mathrm{H}]^{+}: 357.1855$, found: 357.1853 .

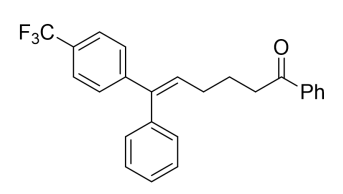

(E)-1,6-Diphenyl-6-(4-(trifluoromethyl)phenyl)hex-5-en-1-one (3f): 109 mg, 92\% yield, white solid, m.p.: 60-62 ${ }^{\circ} \mathrm{C} .{ }^{1} \mathrm{H}$ NMR (400 MHz, $\left.\mathrm{CDCl}_{3}\right) \delta 7.92(\mathrm{~m}, 2 \mathrm{H}$, aromatic $\mathrm{CH}), 7.43(\mathrm{~m}, 10 \mathrm{H}$, aromatic $\mathrm{CH}), 7.15(\mathrm{~m}, 2 \mathrm{H}$, aromatic $\mathrm{CH}), 6.20(\mathrm{t}, J=$ $7.5 \mathrm{~Hz}, 1 \mathrm{H}, \mathrm{C}=\mathrm{CH}), 2.96\left(\mathrm{t}, J=7.3 \mathrm{~Hz}, 2 \mathrm{H}, \mathrm{PhCOCH}_{2}\right), 2.27(\mathrm{dd}, J=14.9$ and 7.5 $\left.\mathrm{Hz}, 2 \mathrm{H}, \mathrm{C}=\mathrm{CHCH}_{2}\right), 1.93\left(\mathrm{~m}, 2 \mathrm{H}, \mathrm{C}=\mathrm{CHCH}_{2} \mathrm{CH}_{2}\right) .{ }^{13} \mathrm{C}\left\{{ }^{1} \mathrm{H}\right\} \mathrm{NMR}(100 \mathrm{MHz}$, $\left.\mathrm{CDCl}_{3}\right) \delta 200.0(\mathrm{Cq}, \mathrm{C}=\mathrm{O}), 146.2,141.5,139.3$, and 137.1 (Cq), 133.1, 131.3, and 129.9 (aromatic $\mathrm{CH}), 129.0$ (q, $J=32.4 \mathrm{~Hz}, \mathrm{Cq}), 128.7,128.5,128.1,127.5$, and 127.5 (aromatic $\mathrm{CH}), 124.4\left(\mathrm{q}, J=270.1 \mathrm{~Hz}, C F_{3}\right), 125.2(\mathrm{q}, J=3.7 \mathrm{~Hz}$, aromatic $\mathrm{CH}), 38.1\left(\mathrm{PhCOCH}_{2}\right), 29.6\left(\mathrm{C}=\mathrm{CHCH}_{2}\right), 24.3\left(\mathrm{C}=\mathrm{CHCH}_{2} \mathrm{CH}_{2}\right)$. HRMS Calcd for $\mathrm{C}_{25} \mathrm{H}_{21} \mathrm{~F}_{3} \mathrm{O}[\mathrm{M}+\mathrm{Na}]^{+}:$417.1442, found: 417.1438 . 


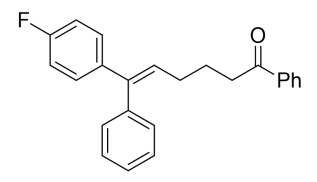

(E)-6-(4-Fluorophenyl)-1,6-diphenylhex-5-en-1-one (3g): $101 \mathrm{mg}, 94 \%$ yield, pale yellow solid, m.p.: $81-83{ }^{\circ} \mathrm{C} .{ }^{1} \mathrm{H}$ NMR $\left(400 \mathrm{MHz}, \mathrm{CDCl}_{3}\right) \delta 7.93(\mathrm{dd}, J=5.1$ and $3.4 \mathrm{~Hz}, 2 \mathrm{H}$, aromatic $\mathrm{CH}), 7.56(\mathrm{~m}, 1 \mathrm{H}$, aromatic $\mathrm{CH}), 7.45(\mathrm{dd}, J=10.5$ and $4.7 \mathrm{~Hz}$, $2 \mathrm{H}$, aromatic $\mathrm{CH}), 7.34(\mathrm{~m}, 3 \mathrm{H}$, aromatic $\mathrm{CH}), 7.18(\mathrm{~m}, 4 \mathrm{H}$, aromatic $\mathrm{CH}), 6.95(\mathrm{~m}$, $2 \mathrm{H}$, aromatic $\mathrm{CH}), 6.07(\mathrm{t}, J=7.5 \mathrm{~Hz}, 1 \mathrm{H}, \mathrm{C}=\mathrm{CH}), 2.96(\mathrm{t}, J=7.4 \mathrm{~Hz}, 2 \mathrm{H}$, PhCOCH$\left.H_{2}\right), 2.24\left(\mathrm{dd}, J=14.9\right.$ and $\left.7.4 \mathrm{~Hz}, 2 \mathrm{H}, \mathrm{C}=\mathrm{CHCH}_{2}\right), 1.92(\mathrm{~m}, 2 \mathrm{H}$, $\left.\mathrm{C}=\mathrm{CHCH}_{2} \mathrm{CH}_{2}\right) .{ }^{13} \mathrm{C}\left\{{ }^{1} \mathrm{H}\right\} \mathrm{NMR}\left(100 \mathrm{MHz}, \mathrm{CDCl}_{3}\right) \delta 200.1(\mathrm{Cq}, \mathrm{C}=\mathrm{O}), 162.1(\mathrm{~d}, J=$ $246.1 \mathrm{~Hz}, \mathrm{Cq}), 141.5$ and $140.0(\mathrm{Cq}), 138.84(\mathrm{~d}, J=3.2 \mathrm{~Hz}, \mathrm{Cq}), 137.1(\mathrm{Cq}), 133.0$ and 129.9 (aromatic $\mathrm{CH}), 129.0(\mathrm{~d}, J=1.3 \mathrm{~Hz}$, aromatic $\mathrm{CH}), 128.8(\mathrm{~d}, J=7.9 \mathrm{~Hz}$, aromatic $\mathrm{CH}), 128.6,128.4(\mathrm{~s}), 128.1(\mathrm{~s})$, and 127.2 (aromatic $\mathrm{CH}), 115.9(\mathrm{~d}, J=21.3$ $\mathrm{Hz}$, aromatic $\mathrm{CH}), 38.1\left(\mathrm{PhCOCH}_{2}\right), 29.4\left(\mathrm{C}=\mathrm{CHCH}_{2}\right), 24.5\left(\mathrm{C}=\mathrm{CHCH}_{2} \mathrm{CH}_{2}\right)$. HRMS Calcd for $\mathrm{C}_{24} \mathrm{H}_{21} \mathrm{FO}[\mathrm{M}+\mathrm{H}]^{+}: 345.1655$, found: 4345.1655 .

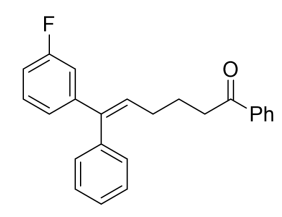

(E)-6-(3-Fluorophenyl)-1,6-diphenylhex-5-en-1-one (3h): $100 \mathrm{mg}, 93 \%$ yield, pale yellow solid, m.p.: 60-62 ${ }^{\circ} \mathrm{C} .{ }^{1} \mathrm{H}$ NMR (400 MHz, $\left.\mathrm{CDCl}_{3}\right) \delta 7.93$ (m, $2 \mathrm{H}$, aromatic $\mathrm{CH}), 7.56(\mathrm{~m}, 1 \mathrm{H}$, aromatic $\mathrm{CH}), 7.45(\mathrm{~m}, 2 \mathrm{H}$, aromatic $\mathrm{CH}), 7.35(\mathrm{~m}, 3 \mathrm{H}$, aromatic $\mathrm{CH}), 7.19(\mathrm{~m}, 3 \mathrm{H}$, aromatic $\mathrm{CH}), 7.02(\mathrm{~m}, 1 \mathrm{H}$, aromatic $\mathrm{CH}), 6.92(\mathrm{~m}, 2 \mathrm{H}$, aromatic $\mathrm{CH}$ ), 6.16 (t, $J=7.5 \mathrm{~Hz}, 1 \mathrm{H}, \mathrm{C}=\mathrm{CH}), 2.96$ (t, $J=7.3 \mathrm{~Hz}, 2 \mathrm{H}, \mathrm{PhCOCH}_{2}$ ), $2.24\left(\mathrm{dd}, J=14.9\right.$ and $\left.7.5 \mathrm{~Hz}, 2 \mathrm{H}, \mathrm{C}=\mathrm{CHCH}_{2}\right), 1.92\left(\mathrm{~m}, 2 \mathrm{H}, \mathrm{C}=\mathrm{CHCH}_{2} \mathrm{CH}_{2}\right)$. ${ }^{13} \mathrm{C}\left\{{ }^{1} \mathrm{H}\right\} \mathrm{NMR}\left(100 \mathrm{MHz}, \mathrm{CDCl}_{3}\right) \delta 200.0(\mathrm{Cq}, \mathrm{C}=\mathrm{O}), 162.9(\mathrm{~d}, J=244.9 \mathrm{~Hz}, \mathrm{Cq})$, $145.0(\mathrm{~d}, J=7.4 \mathrm{~Hz}, \mathrm{Cq}), 141.5(\mathrm{~d}, J=2.3 \mathrm{~Hz}, \mathrm{Cq}), 139.5$ and 137.05 (Cq), 133.0, 130.2, and 129.9 (aromatic $\mathrm{CH}), 129.5(\mathrm{~d}, J=8.4 \mathrm{~Hz}$, aromatic $\mathrm{CH}$ ), 128.6, 128.4, 128.1, and 127.3 (aromatic $\mathrm{CH}), 122.9(\mathrm{~d}, J=2.6 \mathrm{~Hz}$, aromatic $\mathrm{CH}), 114.1(\mathrm{~d}, J=$ 22.0 Hz, aromatic $\mathrm{CH}), 113.8(\mathrm{~d}, J=21.3 \mathrm{~Hz}$, aromatic $\mathrm{CH}), 38.1\left(\mathrm{PhCOCH}_{2}\right), 29.4$ $\left(\mathrm{C}=\mathrm{CHCH}_{2}\right), 24.4\left(\mathrm{C}=\mathrm{CHCH}_{2} \mathrm{CH}_{2}\right)$. HRMS Calcd for $\mathrm{C}_{24} \mathrm{H}_{21} \mathrm{FO}[\mathrm{M}+\mathrm{Na}]^{+}$: 367.1474, 
found: 367.1479 .



(E)-6-(4-Chlorophenyl)-1,6-diphenylhex-5-en-1-one (3i): 99 mg, 92\% yield, pale yellow solid, m.p.: $62-64{ }^{\circ} \mathrm{C} .{ }^{1} \mathrm{H}$ NMR $\left(400 \mathrm{MHz}, \mathrm{CDCl}_{3}\right) \delta 7.92(\mathrm{dd}, J=5.2$ and $3.3 \mathrm{~Hz}, 2 \mathrm{H}$, aromatic $\mathrm{CH}), 7.55(\mathrm{~m}, 1 \mathrm{H}$, aromatic $\mathrm{CH}), 7.45(\mathrm{dd}, J=10.5$ and $4.7 \mathrm{~Hz}$, $2 \mathrm{H}$, aromatic $\mathrm{CH}), 7.34(\mathrm{~m}, 3 \mathrm{H}$, aromatic $\mathrm{CH}), 7.22(\mathrm{~m}, 2 \mathrm{H}$, aromatic $\mathrm{CH}), 7.16(\mathrm{~m}$, $4 \mathrm{H}$, aromatic $\mathrm{CH}), 6.11(\mathrm{t}, J=7.5 \mathrm{~Hz}, 1 \mathrm{H}, \mathrm{C}=\mathrm{CH}), 2.95(\mathrm{t}, J=7.3 \mathrm{~Hz}, 2 \mathrm{H}$, $\left.\mathrm{PhCOCH}_{2}\right), 2.24\left(\mathrm{dd}, J=14.9\right.$ and $\left.7.5 \mathrm{~Hz}, 2 \mathrm{H}, \mathrm{C}=\mathrm{CHCH}_{2}\right), 1.91(\mathrm{p}, J=7.4 \mathrm{~Hz}, 2 \mathrm{H}$, $\left.\mathrm{C}=\mathrm{CHCH}_{2} \mathrm{CH}_{2}\right) .{ }^{13} \mathrm{C}\left\{{ }^{1} \mathrm{H}\right\} \mathrm{NMR}\left(100 \mathrm{MHz}, \mathrm{CDCl}_{3}\right) \delta 200.0(\mathrm{Cq}, \mathrm{C}=\mathrm{O}), 141.4,141.2$, 139.7, and 137.0 (Cq), 133.0 (aromatic CH), $132.8(\mathrm{Cq}), 129.9,129.6,128.7,128.6$, 128.4, 128.3, 128.1, and 127.3 (aromatic $\mathrm{CH}), 38.1\left(\mathrm{PhCOCH}_{2}\right), 29.5\left(\mathrm{C}=\mathrm{CHCH}_{2}\right)$, $24.4\left(\mathrm{C}=\mathrm{CHCH}_{2} \mathrm{CH}_{2}\right)$. HRMS Calcd for $\mathrm{C}_{24} \mathrm{H}_{21} \mathrm{ClO}[\mathrm{M}+\mathrm{H}]^{+}$: 361.1359, found: 361.1361 .

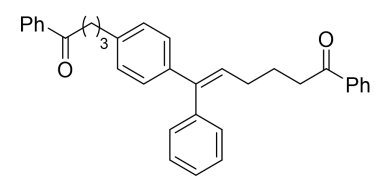

(E)-6-(4-(2-Oxo-2-phenylethyl)phenyl)-1,6-diphenylhex-5-en-1-one (3j): 123 mg, 87\% yield, white solid, m.p.: 107-109 ${ }^{\circ} \mathrm{C} .{ }^{1} \mathrm{H}$ NMR $\left(400 \mathrm{MHz}, \mathrm{CDCl}_{3}\right) \delta 7.94(\mathrm{~m}$, $4 \mathrm{H}$, aromatic $\mathrm{CH}), 7.55(\mathrm{t}, J=7.2 \mathrm{~Hz}, 2 \mathrm{H}$, aromatic $\mathrm{CH}), 7.45(\mathrm{t}, J=7.6 \mathrm{~Hz}, 4 \mathrm{H}$, aromatic $\mathrm{CH}), 7.35(\mathrm{~m}, 3 \mathrm{H}$, aromatic $\mathrm{CH}), 7.16(\mathrm{~m}, 6 \mathrm{H}$, aromatic $\mathrm{CH}), 6.13(\mathrm{t}, J=$ $7.4 \mathrm{~Hz}, 1 \mathrm{H}, \mathrm{C}=\mathrm{CH}), 2.98\left(\mathrm{~m}, 4 \mathrm{H}, 2 \times \mathrm{PhCOCH}_{2}\right), 2.72(\mathrm{t}, J=7.5 \mathrm{~Hz}, 2 \mathrm{H}$, $\mathrm{PhCOCH}_{2} \mathrm{CH}_{2} \mathrm{CH}_{2}$ ), 2.25 (dd, $J=14.8$ and $\left.7.4 \mathrm{~Hz}, 2 \mathrm{H}, \mathrm{C}=\mathrm{CHCH}_{2}\right), 2.09$ (m, $2 \mathrm{H}$, $\left.\mathrm{PhCOCH}_{2} \mathrm{CH}_{2} \mathrm{CH}_{2}\right), 1.92\left(\mathrm{~m}, 2 \mathrm{H}, \mathrm{C}=\mathrm{CHCH}_{2} \mathrm{CH}_{2}\right) \cdot{ }^{13} \mathrm{C}\left\{{ }^{1} \mathrm{H}\right\} \mathrm{NMR}\left(100 \mathrm{MHz}, \mathrm{CDCl}_{3}\right)$ $\delta 200.1(\mathrm{Cq}, 2 \times \mathrm{C}=\mathrm{O}), 142.2,140.5,140.3,140.2$, and $137.0(\mathrm{Cq}), 133.0,132.9,129.9$, 128.6, 128.6, 128.4, 128.3, 128.2, 128.0, 127.2, and 127.0 (aromatic $\mathrm{CH}$ ), 38.1 and $37.7\left(2 \times \mathrm{PhCOCH}_{2}\right), \quad 34.8 \quad\left(\mathrm{PhCOCH}_{2} \mathrm{CH}_{2} \mathrm{CH}_{2}\right), \quad 29.3 \quad\left(\mathrm{C}=\mathrm{CHCH}_{2}\right), \quad 25.7$ $\left(\mathrm{PhCOCH}_{2} \mathrm{CH}_{2} \mathrm{CH}_{2}\right), 24.5\left(\mathrm{C}=\mathrm{CHCH}_{2} \mathrm{CH}_{2}\right)$. HRMS Calcd for $\mathrm{C}_{34} \mathrm{H}_{32} \mathrm{O}_{2}[\mathrm{M}+\mathrm{H}]^{+}$: 473.2481, found: 473.2482 . 


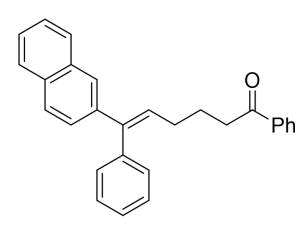

(E)-6-(Naphthalen-2-yl)-1,6-diphenylhex-5-en-1-one (3k): 105 mg, 93\% yield, pale yellow solid, m.p.: 117-119 ${ }^{\circ} \mathrm{C} .{ }^{1} \mathrm{H}$ NMR $\left(400 \mathrm{MHz}, \mathrm{CDCl}_{3}\right) \delta 7.96(\mathrm{~m}, 2 \mathrm{H}$, aromatic $\mathrm{CH}), 7.78(\mathrm{~m}, 3 \mathrm{H}$, aromatic $\mathrm{CH}), 7.46(\mathrm{~m}, 10 \mathrm{H}$, aromatic $\mathrm{CH}), 7.26(\mathrm{~m}, 2$ $\mathrm{H}$, aromatic $\mathrm{CH}), 6.29(\mathrm{t}, J=7.4 \mathrm{~Hz}, 1 \mathrm{H}, \mathrm{C}=\mathrm{CH}), 2.99(\mathrm{t}, J=7.4 \mathrm{~Hz}, 2 \mathrm{H}$, $\mathrm{PhCOCH}_{2}$ ), $2.32\left(\mathrm{q}, J=7.4 \mathrm{~Hz}, 2 \mathrm{H}, \mathrm{C}=\mathrm{CHCH}_{2}\right), 1.98\left(\mathrm{~m}, 2 \mathrm{H}, \mathrm{C}=\mathrm{CHCH}_{2} \mathrm{CH}_{2}\right.$ ). ${ }^{13} \mathrm{C}\left\{{ }^{1} \mathrm{H}\right\} \mathrm{NMR}\left(100 \mathrm{MHz}, \mathrm{CDCl}_{3}\right) \delta 200.1(\mathrm{Cq}, \mathrm{C}=\mathrm{O}), 142.5,140.1,140.0,137.1$, and $133.4(\mathrm{Cq}), 133.0$ (aromatic CH), $132.6(\mathrm{Cq}), 130.1,129.7,128.6,128.4,128.2,128.1$, 127.7, 127.6, 127.2, 126.4, 126.1, 125.8, and 125.4 (aromatic $\mathrm{CH}), 38.1\left(\mathrm{PhCOCH}_{2}\right)$, $29.6\left(\mathrm{C}=\mathrm{CHCH}_{2}\right), 24.5\left(\mathrm{C}=\mathrm{CHCH}_{2} \mathrm{CH}_{2}\right)$. HRMS Calcd for $\mathrm{C}_{28} \mathrm{H}_{24} \mathrm{O} \quad[\mathrm{M}+\mathrm{H}]^{+}$: 377.1905, found: 377.1904 .

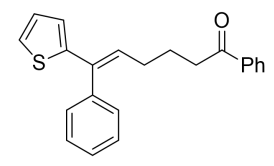

(E)-1,6-Diphenyl-6-(thiophen-2-yl)hex-5-en-1-one (3l): $43 \mathrm{mg}, 43 \%$ yield, yellow solid, m.p.: $55-57{ }^{\circ} \mathrm{C} .{ }^{1} \mathrm{H}$ NMR $\left(400 \mathrm{MHz}, \mathrm{CDCl}_{3}\right) \delta 7.86(\mathrm{~m}, 2 \mathrm{H}$, aromatic $\mathrm{CH}), 7.49(\mathrm{t}, J=7.4 \mathrm{~Hz}, 1 \mathrm{H}$, aromatic $\mathrm{CH}), 7.32(\mathrm{~m}, 5 \mathrm{H}$, aromatic $\mathrm{CH}), 7.18(\mathrm{~m}, 2 \mathrm{H}$, aromatic $\mathrm{CH}), 7.08(\mathrm{~m}, 1 \mathrm{H}$, aromatic $\mathrm{CH}), 6.83(\mathrm{dd}, J=5.1$ and $3.7 \mathrm{~Hz}, 1 \mathrm{H}$, aromatic $\mathrm{CH}), 6.52(\mathrm{~m}, 1 \mathrm{H}$, aromatic $\mathrm{CH}), 6.13(\mathrm{t}, J=7.5 \mathrm{~Hz}, 1 \mathrm{H}, \mathrm{C}=\mathrm{CH}), 2.87(\mathrm{t}, J$ $\left.=7.4 \mathrm{~Hz}, 2 \mathrm{H}, \mathrm{PhCOCH}_{2}\right), 2.09$ (q, $\left.J=7.4 \mathrm{~Hz}, 2 \mathrm{H}, \mathrm{C}=\mathrm{CHCH}_{2}\right), 1.82(\mathrm{~m}, 2 \mathrm{H}$, $\left.\mathrm{C}=\mathrm{CHCH}_{2} \mathrm{CH}_{2}\right) .{ }^{13} \mathrm{C}\left\{{ }^{1} \mathrm{H}\right\} \mathrm{NMR}\left(100 \mathrm{MHz}, \mathrm{CDCl}_{3}\right) \delta 200.1(\mathrm{Cq}, \mathrm{C}=\mathrm{O}), 147.1,139.2$, 137.1, and 136.7 (Cq), 133.0, 129.6, 128.6, 128.4, 128.1, 127.8, 127.5, 127.3, 125.1, and 123.9 (aromatic $\mathrm{CH}), 38.0\left(\mathrm{PhCOCH}_{2}\right), 29.0\left(\mathrm{C}=\mathrm{CHCH}_{2}\right), 24.3\left(\mathrm{C}=\mathrm{CHCH}_{2} \mathrm{CH}_{2}\right)$. HRMS Calcd for $\mathrm{C}_{22} \mathrm{H}_{20} \mathrm{OS}[\mathrm{M}+\mathrm{H}]^{+}: 333.1313$, found: 333.1312 .



(Z)-6-(4-Methoxyphenyl)-1,6-diphenylhex-5-en-1-one (3m): 99 mg, 92\% yield, white solid, m.p.: 90-92 ${ }^{\circ} \mathrm{C} .{ }^{1} \mathrm{H}$ NMR $\left(400 \mathrm{MHz}, \mathrm{CDCl}_{3}\right) \delta 7.99$ (m, $2 \mathrm{H}$, aromatic 
$\mathrm{CH}), 7.57(\mathrm{~m}, 3 \mathrm{H}$, aromatic $\mathrm{CH}), 7.31(\mathrm{~m}, 5 \mathrm{H}$, aromatic $\mathrm{CH}), 7.16(\mathrm{~m}, 2 \mathrm{H}$, aromatic $\mathrm{CH}), 6.96(\mathrm{~m}, 2 \mathrm{H}$, aromatic $\mathrm{CH}), 6.14(\mathrm{t}, J=7.4 \mathrm{~Hz}, 1 \mathrm{H}, \mathrm{C}=\mathrm{CH}), 3.89(\mathrm{~s}, 3 \mathrm{H}$, $\left.\mathrm{OCH}_{3}\right), 3.02$ (t, $\left.J=7.4 \mathrm{~Hz}, 2 \mathrm{H}, \mathrm{PhCOCH}_{2}\right), 2.33$ (dd, $J=14.9$ and $7.4 \mathrm{~Hz}, 2 \mathrm{H}$, $\left.\mathrm{C}=\mathrm{CHCH}_{2}\right), 1.98\left(\mathrm{~m}, 2 \mathrm{H}, \mathrm{C}=\mathrm{CHCH}_{2} \mathrm{CH}_{2}\right) .{ }^{13} \mathrm{C}\left\{{ }^{1} \mathrm{H}\right\} \mathrm{NMR}\left(100 \mathrm{MHz}, \mathrm{CDCl}_{3}\right) \delta 200.1$ $(\mathrm{Cq}, \mathrm{C}=\mathrm{O}), 158.6,143.1,142.1$, and $137.1(\mathrm{Cq}), 133.0$ (aromatic $\mathrm{CH}), 132.4(\mathrm{Cq})$, 131.1, 128.8, 128.6, 128.1, 128.1, 127.4, 126.9, and 113.6 (aromatic $\mathrm{CH}$ ), 55.2 $\left(\mathrm{OCH}_{3}\right), 38.1\left(\mathrm{PhCOCH}_{2}\right), 29.4\left(\mathrm{C}=\mathrm{CHCH}_{2}\right), 24.5\left(\mathrm{C}=\mathrm{CHCH}_{2} \mathrm{CH}_{2}\right)$. HRMS Calcd for $\mathrm{C}_{25} \mathrm{H}_{24} \mathrm{O}_{2}[\mathrm{M}+\mathrm{H}]^{+}:$357.1855, found: 357.1853 .

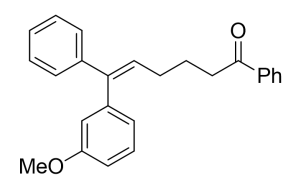

(Z)-6-(3-Methoxyphenyl)-1,6-diphenylhex-5-en-1-one (3n): 96 mg, 90\% yield, pale yellow solid, m.p.: $48-50{ }^{\circ} \mathrm{C} .{ }^{1} \mathrm{H}$ NMR (400 MHz, $\left.\mathrm{CDCl}_{3}\right) \delta 7.89(\mathrm{~m}, 2 \mathrm{H}$, aromatic $\mathrm{CH}), 7.50(\mathrm{~m}, 1 \mathrm{H}$, aromatic $\mathrm{CH}), 7.40(\mathrm{dd}, J=10.5$ and $4.6 \mathrm{~Hz}, 2 \mathrm{H}$, aromatic $\mathrm{CH}), 7.20(\mathrm{~m}, 6 \mathrm{H}$, aromatic $\mathrm{CH}), 6.83(\mathrm{~m}, 1 \mathrm{H}$, aromatic $\mathrm{CH}), 6.70(\mathrm{~m}, 2 \mathrm{H}$, aromatic $\mathrm{CH}), 6.08(\mathrm{t}, J=7.5 \mathrm{~Hz}, 1 \mathrm{H}, \mathrm{C}=\mathrm{CH}), 3.74\left(\mathrm{~s}, 3 \mathrm{H}, \mathrm{OCH}_{3}\right), 2.92(\mathrm{t}, J=7.4$ $\left.\mathrm{Hz}, 2 \mathrm{H}, \mathrm{PhCOCH}{ }_{2}\right), 2.21$ (q, $\left.J=7.4 \mathrm{~Hz}, 2 \mathrm{H}, \mathrm{C}=\mathrm{CHCH}_{2}\right), 1.87$ (p, $J=7.5 \mathrm{~Hz}, 2 \mathrm{H}$, $\left.\mathrm{C}=\mathrm{CHCH}_{2} \mathrm{CH}_{2}\right) .{ }^{13} \mathrm{C}\left\{{ }^{1} \mathrm{H}\right\} \mathrm{NMR}\left(100 \mathrm{MHz}, \mathrm{CDCl}_{3}\right) \delta 200.1(\mathrm{Cq}, \mathrm{C}=\mathrm{O}), 159.6,142.3$, 142.3, 141.5, and 137.1 (Cq), 133.0, 129.3, 129.0, 128.6, 128.2, 128.1, 127.2, 127.0, 122.4, 115.5, and 112.5 (aromatic $\mathrm{CH}), 55.2\left(\mathrm{OCH}_{3}\right), 38.1\left(\mathrm{PhCOCH}_{2}\right), 29.4$ $\left(\mathrm{C}=\mathrm{CHCH}_{2}\right), 24.5\left(\mathrm{C}=\mathrm{CHCH}_{2} \mathrm{CH}_{2}\right)$. HRMS Calcd for $\mathrm{C}_{25} \mathrm{H}_{24} \mathrm{O}_{2}[\mathrm{M}+\mathrm{Na}]^{+}:$379.1674, found: 379.1675 .

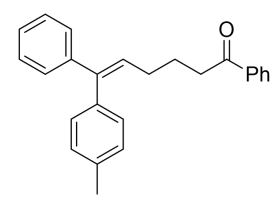

(Z)-1,6-Diphenyl-6-(p-tolyl)hex-5-en-1-one (3o): $92 \mathrm{mg}, 90 \%$ yield, white solid, m.p.: $124-126{ }^{\circ} \mathrm{C} .{ }^{1} \mathrm{H}$ NMR $\left(400 \mathrm{MHz}, \mathrm{CDCl}_{3}\right) \delta 7.90(\mathrm{~m}, 2 \mathrm{H}$, aromatic $\mathrm{CH}), 7.52(\mathrm{t}$, $J=7.4 \mathrm{~Hz}, 1 \mathrm{H}$, aromatic $\mathrm{CH}), 7.42(\mathrm{t}, J=7.6 \mathrm{~Hz}, 2 \mathrm{H}$, aromatic $\mathrm{CH}), 7.20(\mathrm{~m}, 7 \mathrm{H}$, aromatic $\mathrm{CH}), 7.04(\mathrm{~d}, J=7.9 \mathrm{~Hz}, 2 \mathrm{H}$, aromatic $\mathrm{CH}), 6.07$ (t, $J=7.4 \mathrm{~Hz}, 1 \mathrm{H}$, $\mathrm{C}=\mathrm{C} H), 2.93\left(\mathrm{t}, J=7.4 \mathrm{~Hz}, 2 \mathrm{H}, \mathrm{PhCOCH}_{2}\right), 2.37\left(\mathrm{~s}, 3 \mathrm{H}, \mathrm{CH}_{3}\right), 2.23(\mathrm{dd}, J=14.8$ 
and $\left.7.4 \mathrm{~Hz}, 2 \mathrm{H}, \mathrm{C}=\mathrm{CHCH}_{2}\right), 1.89\left(\mathrm{~m}, 2 \mathrm{H}, \mathrm{C}=\mathrm{CHCH}_{2} \mathrm{CH}_{2}\right) .{ }^{13} \mathrm{C}\left\{{ }^{1} \mathrm{H}\right\} \mathrm{NMR}(100$ $\left.\mathrm{MHz}, \mathrm{CDCl}_{3}\right) \delta 200.1(\mathrm{Cq}, \mathrm{C}=\mathrm{O}), 142.9,142.4,137.1,137.1$, and 136.6 (aromatic $\mathrm{CH}), 133.0,129.9,129.0,128.9,128.6,128.1,128.1,127.3$, and 126.9 (aromatic $\mathrm{CH}$ ), $38.1\left(\mathrm{PhCOCH}_{2}\right), 29.4\left(\mathrm{C}=\mathrm{CHCH}_{2}\right), 24.5\left(\mathrm{C}=\mathrm{CHCH}_{2} \mathrm{CH}_{2}\right), 21.3\left(\mathrm{CH}_{3}\right)$. HRMS Calcd for $\mathrm{C}_{25} \mathrm{H}_{24} \mathrm{O}[\mathrm{M}+\mathrm{H}]^{+}: 341.1905$, found: 341.1902 .

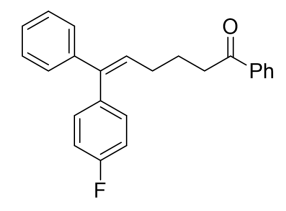

(Z)-6-(4-Fluorophenyl)-1,6-diphenylhex-5-en-1-one (3p): $93 \mathrm{mg}, 90 \%$ yield, white solid, m.p.: 98-100 ${ }^{\circ} \mathrm{C} .{ }^{1} \mathrm{H}$ NMR $\left(400 \mathrm{MHz}, \mathrm{CDCl}_{3}\right) \delta 7.91$ (m, $2 \mathrm{H}$, aromatic $\mathrm{CH}), 7.54(\mathrm{~m}, 1 \mathrm{H}$, aromatic $\mathrm{CH}), 7.43(\mathrm{dd}, J=10.5$ and $4.7 \mathrm{~Hz}, 2 \mathrm{H}$, aromatic $\mathrm{CH})$, $7.23(\mathrm{~m}, 5 \mathrm{H}$, aromatic $\mathrm{CH}), 7.10(\mathrm{~m}, 2 \mathrm{H}$, aromatic $\mathrm{CH}), 7.03(\mathrm{~m}, 2 \mathrm{H}$, aromatic $\mathrm{CH})$, $6.10(\mathrm{t}, J=7.5 \mathrm{~Hz}, 1 \mathrm{H}, \mathrm{C}=\mathrm{CH}), 2.94\left(\mathrm{t}, J=7.3 \mathrm{~Hz}, 2 \mathrm{H}, \mathrm{PhCOCH}_{2}\right), 2.21(\mathrm{dd}, J=$ 14.9 and $\left.7.5 \mathrm{~Hz}, 2 \mathrm{H}, \mathrm{C}=\mathrm{CHCH}_{2}\right), 1.90\left(\mathrm{~m}, 2 \mathrm{H}, \mathrm{C}=\mathrm{CHCH}_{2} \mathrm{CH}_{2}\right) .{ }^{13} \mathrm{C}\left\{{ }^{1} \mathrm{H}\right\} \mathrm{NMR}(100$ $\left.\mathrm{MHz}, \mathrm{CDCl}_{3}\right) \delta 200.0(\mathrm{Cq}, \mathrm{C}=\mathrm{O}), 161.9(\mathrm{~d}, J=245.8 \mathrm{~Hz}, \mathrm{Cq}), 142.5,141.5$, and $137.1(\mathrm{Cq}), 135.97$ (d, $J=3.5 \mathrm{~Hz}, \mathrm{Cq}), 133.0$ (aromatic $\mathrm{CH}), 131.5$ (d, $J=7.9 \mathrm{~Hz}$, aromatic $\mathrm{CH}), 129.5,128.7,128.3,128.1,127.3$, and 127.2 (aromatic $\mathrm{CH}), 115.23(\mathrm{~d}$, $J=21.2 \mathrm{~Hz}$, aromatic $\mathrm{CH}), 38.0\left(\mathrm{PhCOCH}_{2}\right), 29.4\left(\mathrm{C}=\mathrm{CHCH}_{2}\right), 24.4\left(\mathrm{C}=\mathrm{CHCH}_{2} \mathrm{CH}_{2}\right)$ HRMS Calcd for $\mathrm{C}_{24} \mathrm{H}_{21} \mathrm{FO}[\mathrm{M}+\mathrm{Na}]^{+}: 367.1474$, found: 367.1473 .

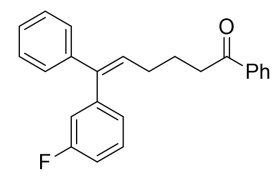

(Z)-6-(3-Fluorophenyl)-1,6-diphenylhex-5-en-1-one (3q): $89 \mathrm{mg}$, 86\% yield, pale solid, m.p.: $78-80{ }^{\circ} \mathrm{C} .{ }^{1} \mathrm{H}$ NMR $\left(400 \mathrm{MHz} \mathrm{CDCl}_{3}\right) \delta 7.96(\mathrm{~m}, 2 \mathrm{H}$, aromatic $\mathrm{CH})$, $7.55(\mathrm{~m}, 3 \mathrm{H}$, aromatic $\mathrm{CH}), 7.30(\mathrm{~m}, 6 \mathrm{H}$, aromatic $\mathrm{CH}), 6.98(\mathrm{~m}, 3 \mathrm{H}$, aromatic $\mathrm{CH})$, $6.17(\mathrm{t}, J=7.5 \mathrm{~Hz}, 1 \mathrm{H}, \mathrm{C}=\mathrm{CH}), 2.99\left(\mathrm{t}, J=7.3 \mathrm{~Hz}, 2 \mathrm{H}, \mathrm{PhCOCH}_{2}\right), 2.27(\mathrm{dd}, J=$ 14.9 and $\left.7.5 \mathrm{~Hz}, 2 \mathrm{H}, \mathrm{C}=\mathrm{CHCH}_{2}\right), 1.95\left(\mathrm{~m}, 2 \mathrm{H}, \mathrm{C}=\mathrm{CHCH}_{2} \mathrm{CH}_{2}\right) .{ }^{13} \mathrm{C}\left\{{ }^{1} \mathrm{H}\right\} \mathrm{NMR}(100$ $\left.\mathrm{MHz}, \mathrm{CDCl}_{3}\right) \delta 200.0(\mathrm{Cq}, \mathrm{C}=\mathrm{O}), 162.8(\mathrm{~d}, J=246.2 \mathrm{~Hz}, \mathrm{Cq}), 142.4(\mathrm{~d}, J=7.5 \mathrm{~Hz}$, Cq), $142.0(\mathrm{Cq}), 141.4(\mathrm{~d}, J=1.7 \mathrm{~Hz}, \mathrm{Cq}), 137.0(\mathrm{Cq}), 133.1,129.8,129.8,128.7$, 128.3, 128.1, 127.3, and 127.2 (aromatic $\mathrm{CH}), 125.75(\mathrm{~d}, J=2.8 \mathrm{~Hz}$, aromatic $\mathrm{CH}$ ), 
$116.9(\mathrm{~d}, J=21.0 \mathrm{~Hz}$, aromatic $\mathrm{CH}), 114.0(\mathrm{~d}, J=21.0 \mathrm{~Hz}$, aromatic $\mathrm{CH}), 38.0$ $\left(\mathrm{PhCOCH}_{2}\right), 29.4\left(\mathrm{C}=\mathrm{CHCH}_{2}\right), 24.4\left(\mathrm{C}=\mathrm{CHCH}_{2} \mathrm{CH}_{2}\right)$. HRMS Calcd for $\mathrm{C}_{24} \mathrm{H}_{21} \mathrm{FO}$ $[\mathrm{M}+\mathrm{Na}]^{+}: 367.1474$, found: 367.1471 .

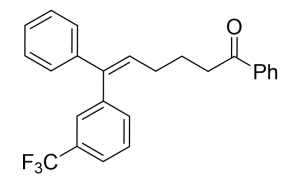

(Z)-1,6-Diphenyl-6-(3-(trifluoromethyl)phenyl)hex-5-en-1-one (3r): $105.6 \mathrm{mg}$, 89\% yield, yellow liquid. ${ }^{1} \mathrm{H}$ NMR $\left(400 \mathrm{MHz}, \mathrm{CDCl}_{3}\right) \delta 7.94(\mathrm{~m}, 2 \mathrm{H}$, aromatic $\mathrm{CH})$, $7.60(\mathrm{~m}, 2 \mathrm{H}$, aromatic CH), $7.48(\mathrm{~m}, 4 \mathrm{H}$, aromatic $\mathrm{CH}), 7.39(\mathrm{~d}, J=7.6 \mathrm{~Hz}, 1 \mathrm{H}$, aromatic $\mathrm{CH}), 7.30(\mathrm{~m}, 3 \mathrm{H}$, aromatic $\mathrm{CH}), 7.22(\mathrm{~m}, 2 \mathrm{H}$, aromatic $\mathrm{CH}), 6.21(\mathrm{t}, J=$ $7.5 \mathrm{~Hz}, 1 \mathrm{H}$, alkenyl CH), $2.99\left(\mathrm{t}, J=7.3 \mathrm{~Hz}, 2 \mathrm{H}, \mathrm{PhCOCH}_{2}\right), 2.24(\mathrm{~m}, 2 \mathrm{H}$, $\left.\mathrm{C}=\mathrm{CHCH}_{2}\right), 2.03-1.85\left(\mathrm{~m}, 2 \mathrm{H}, \mathrm{C}=\mathrm{CHCH}_{2} \mathrm{CH}_{2}\right) .{ }^{13} \mathrm{C}\left\{{ }^{1} \mathrm{H}\right\} \mathrm{NMR}\left(100 \mathrm{MHz}, \mathrm{CDCl}_{3}\right) \delta$ $200.0(\mathrm{Cq}, \mathrm{C}=\mathrm{O}), 141.9,141.2,141.0$, and $137.0(\mathrm{Cq}), 133.5,133.1,130.3,128.9$, 128.7, 128.4, 128.1, 127.4, and 127.3 (aromatic CH), 130.8 (q, $J=31.8 \mathrm{~Hz}, i-\mathrm{C}$ of $\left.\mathrm{CF}_{3} \mathrm{C}_{6} \mathrm{H}_{4}\right), 126.6$ (q, $J=3.8 \mathrm{~Hz}, o-\mathrm{C}$ of $\left.\mathrm{CF}_{3} \mathrm{C}_{6} \mathrm{H}_{4}\right), 124.3\left(\mathrm{q}, J=270.5 \mathrm{~Hz}, \mathrm{CF}_{3}\right), 124.0$ $\left(\mathrm{q}, \quad J=3.7 \mathrm{~Hz}, \quad o-\mathrm{C}\right.$ of $\left.\mathrm{CF}_{3} \mathrm{C}_{6} \mathrm{H}_{4}\right), \quad 38.0\left(\mathrm{PhCOCH}_{2}\right), 29.4 \quad\left(\mathrm{C}=\mathrm{CHCH}_{2}\right), \quad 24.3$ $\left(\mathrm{C}=\mathrm{CHCH}_{2} \mathrm{CH}_{2}\right)$. HRMS Calcd for $\mathrm{C}_{25} \mathrm{H}_{21} \mathrm{~F}_{3} \mathrm{O}[\mathrm{M}+\mathrm{H}]^{+}$: 395.1623, found: 395.1626.

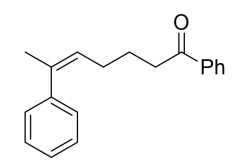

(Z)-1,6-Diphenylhept-5-en-1-one (3s): $70 \mathrm{mg}, 88 \%(10: 1,3 \mathrm{~s}: 4 \mathrm{~s})$ yield, pale yellow liquid. ${ }^{1} \mathrm{H}$ NMR (400 MHz, $\left.\mathrm{CDCl}_{3}\right) \delta 7.84(\mathrm{~m}, 2.2 \mathrm{H}), 7.46(\mathrm{~m}, 1.1 \mathrm{H}), 7.36$ (m, $2.2 \mathrm{H}), 7.24(\mathrm{~m}, 2.2 \mathrm{H}), 7.11(\mathrm{~m}, 3.4 \mathrm{H}), 5.42(\mathrm{td}, J=7.3$ and $1.3 \mathrm{~Hz}, 1 \mathrm{H}), 2.81$ (m, $2 \mathrm{H}), 1.99(\mathrm{~m}, 5.7 \mathrm{H}), 1.71(\mathrm{~m}, 2.2 \mathrm{H}) .{ }^{13} \mathrm{C}\left\{{ }^{1} \mathrm{H}\right\} \mathrm{NMR}\left(100 \mathrm{MHz}, \mathrm{CDCl}_{3}\right) \delta 200.3$, $142.1,137.3,137.1,132.9,128.6,128.2,128.1,128.0,126.9,126.6,38.1,28.7,25.7$, 24.7. HRMS Calcd for $\mathrm{C}_{19} \mathrm{H}_{20} \mathrm{O}[\mathrm{M}+\mathrm{H}]^{+}: 265.1592$, found: 265.1589 .

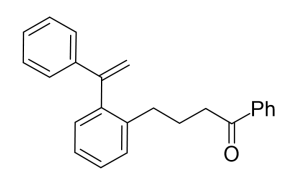

1-Phenyl-4-(2-(1-phenylvinyl)phenyl)butan-1-one (4a): $90 \mathrm{mg}, 92 \%$ yield, colorless liquid. ${ }^{1} \mathrm{H}$ NMR $\left(400 \mathrm{MHz}, \mathrm{CDCl}_{3}\right) \delta 7.71(\mathrm{~m} 2 \mathrm{H}$, aromatic $\mathrm{CH}), 7.39$ (t, J $=7.3 \mathrm{~Hz}, 1 \mathrm{H}$, aromatic $\mathrm{CH}), 7.28(\mathrm{t}, J=7.6 \mathrm{~Hz}, 2 \mathrm{H}$, aromatic $\mathrm{CH}), 7.14(\mathrm{~m}, 9 \mathrm{H}$, 
aromatic $\mathrm{CH}$ ), 5.65 and 5.09 (s each, 1:1 H, C=CH2), $2.65(\mathrm{t}, J=7.4 \mathrm{~Hz}, 2 \mathrm{H}$, $\mathrm{PhCOCH}_{2} \mathrm{CH}_{2} \mathrm{CH}_{2}$ ), 2.38 (t, $\left.J=7.7 \mathrm{~Hz}, 2 \mathrm{H}, \mathrm{PhCOCH}_{2} \mathrm{CH}_{2} \mathrm{CH}_{2}\right), 1.78(\mathrm{~m}, 2 \mathrm{H}$, $\left.\mathrm{PhCOCH}_{2} \mathrm{CH}_{2} \mathrm{CH}_{2}\right) .{ }^{13} \mathrm{C}\left\{{ }^{1} \mathrm{H}\right\} \mathrm{NMR}\left(100 \mathrm{MHz}, \mathrm{CDCl}_{3}\right) \delta 200.0(\mathrm{Cq}, \mathrm{C}=\mathrm{O}), 149.2$, 141.4, 140.9, 139.8, and 137.0 (Cq), 132.9, 130.5, 129.4, 128.5, 128.4, 128.0, 127.8, 127.7, 126.5, and 126.0 (aromatic $\mathrm{CH}), 115.4\left(\mathrm{C}=\mathrm{CH}_{2}\right), 38.2\left(\mathrm{PhCOCH}_{2} \mathrm{CH}_{2} \mathrm{CH}_{2}\right)$, $32.7\left(\mathrm{PhCOCH}_{2} \mathrm{CH}_{2} \mathrm{CH}_{2}\right)$, $25.5\left(\mathrm{PhCOCH}_{2} \mathrm{CH}_{2} \mathrm{CH}_{2}\right)$. HRMS Calcd for $\mathrm{C}_{24} \mathrm{H}_{22} \mathrm{O}$ $[\mathrm{M}+\mathrm{H}]^{+}:$327.1749, found: 327.1750 .



(E)-1-(4-Methoxyphenyl)-6-phenyl-6-(o-tolyl)hex-5-en-1-one (5a): 105 mg, 95\% yield, white solid, m.p.: 91-93 ${ }^{\circ} \mathrm{C} .{ }^{1} \mathrm{H}$ NMR (400 MHz, $\left.\mathrm{CDCl}_{3}\right) \delta 7.88(\mathrm{~d}, J=8.9$ $\mathrm{Hz}, 2 \mathrm{H}$, aromatic $\mathrm{CH}), 7.18(\mathrm{~m}, 8 \mathrm{H}$, aromatic $\mathrm{CH}), 7.07(\mathrm{~m}, 1 \mathrm{H}$, aromatic $\mathrm{CH}), 6.87$ (t, $J=5.8 \mathrm{~Hz}, 2 \mathrm{H}$, aromatic $\mathrm{CH}), 5.68(\mathrm{t}, J=7.4 \mathrm{~Hz}, 1 \mathrm{H}, \mathrm{C}=\mathrm{CH}), 3.80(\mathrm{~s}, 3 \mathrm{H}$, $\left.\mathrm{OCH}_{3}\right), 2.90\left(\mathrm{t}, J=7.4 \mathrm{~Hz}, 2 \mathrm{H}, \mathrm{PhCOCH}_{2}\right), 2.39$ (dd, $J=14.9$ and $7.4 \mathrm{~Hz}, 2 \mathrm{H}$, $\left.\mathrm{C}=\mathrm{CHCH}_{2}\right), 2.01\left(\mathrm{~s}, 3 \mathrm{H}, \mathrm{CH}_{3}\right), 1.89\left(\mathrm{~m}, 2 \mathrm{H}, \mathrm{C}=\mathrm{CHCH}_{2} \mathrm{CH}_{2}\right) .{ }^{13} \mathrm{C}\left\{{ }^{1} \mathrm{H}\right\} \mathrm{NMR}(100$ $\left.\mathrm{MHz}, \mathrm{CDCl}_{3}\right){ }^{13} \mathrm{C} \mathrm{NMR}\left(101 \mathrm{MHz}, \mathrm{CDCl}_{3}\right) \delta 198.7$ (Cq, C=O), 163.4, 143.9, 142.4, 140.2, and 136.1 (Cq), 131.4, 130.3, 130.3, and 130.2 (aromatic CH), $130.2(\mathrm{Cq})$, $129.3,128.0,127.1,126.7,125.6$, and 113.7 (aromatic $\mathrm{CH}$ ), $55.5\left(\mathrm{OCH}_{3}\right), 37.8$ $\left(\mathrm{PhCOCH}_{2}\right), 29.0\left(\mathrm{C}=\mathrm{CHCH}_{2}\right), 24.9\left(\mathrm{C}=\mathrm{CHCH}_{2} \mathrm{CH}_{2}\right), 20.5\left(\mathrm{CH}_{3}\right)$. HRMS Calcd for $\mathrm{C}_{26} \mathrm{H}_{26} \mathrm{O}_{2}[\mathrm{M}+\mathrm{H}]^{+}:$371.2011, found: 371.2015.

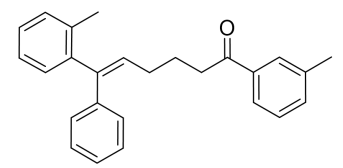

(E)-6-Phenyl-1-(m-tolyl)-6-(o-tolyl)hex-5-en-1-one (5b): $100 \mathrm{mg}$, 94\% yield, pale yellow solid, m.p.: 82-84 ${ }^{\circ} \mathrm{C} .{ }^{1} \mathrm{H}$ NMR (400 MHz, $\left.\mathrm{CDCl}_{3}\right) \delta 7.87(\mathrm{~m}, 2 \mathrm{H}$, aromatic $\mathrm{CH}), 7.45(\mathrm{~m}, 4 \mathrm{H}$, aromatic $\mathrm{CH}), 7.33(\mathrm{~m}, 6 \mathrm{H}$, aromatic $\mathrm{CH}), 7.24(\mathrm{~m}, 1 \mathrm{H}$, aromatic $\mathrm{CH}), 5.86$ (t, $J=7.4 \mathrm{~Hz}, 1 \mathrm{H}, \mathrm{C}=\mathrm{CH}), 3.11\left(\mathrm{t}, J=7.4 \mathrm{~Hz}, 2 \mathrm{H}, \mathrm{PhCOCH}_{2}\right.$ ), $2.57\left(\mathrm{~m}, 5 \mathrm{H}, \mathrm{C}=\mathrm{CHCH}_{2}\right.$ and $\left.\mathrm{CH}_{3}\right), 2.19\left(\mathrm{~s}, 3 \mathrm{H}, \mathrm{CH}_{3}\right), 2.07\left(\mathrm{~m}, 2 \mathrm{H}, \mathrm{C}=\mathrm{CHCH}_{2} \mathrm{CH}_{2}\right)$. ${ }^{13} \mathrm{C}\left\{{ }^{1} \mathrm{H}\right\}$ NMR $\left(100 \mathrm{MHz}, \mathrm{CDCl}_{3}\right) \delta 200.3(\mathrm{Cq}, \mathrm{C}=\mathrm{O}), 143.9,142.4,140.2,138.4$, 137.1, and 136.1 (Cq), 133.7, 131.3, 130.3, 130.2, 129.4, 128.6, 128.5, 128.0, 127.2, 126.7, 125.6, and 125.3 (aromatic $\mathrm{CH}), 38.2\left(\mathrm{PhCOCH}_{2}\right), 28.9\left(\mathrm{C}=\mathrm{CHCH}_{2}\right), 24.8$ 
$\left(\mathrm{C}=\mathrm{CHCH}_{2} \mathrm{CH}_{2}\right), 21.4\left(\mathrm{CH}_{3}\right), 20.5\left(\mathrm{CH}_{3}\right)$. HRMS Calcd for $\mathrm{C}_{26} \mathrm{H}_{26} \mathrm{O}[\mathrm{M}+\mathrm{H}]^{+}$: 355.2062, found: 355.2060 .



(E)-6-Phenyl-1,6-di-o-tolylhex-5-en-1-one (5c): $98 \mathrm{mg}$, 92\% yield, pale yellow solid, m.p.: 64-66 ${ }^{\circ} \mathrm{C} .{ }^{1} \mathrm{H}$ NMR $\left(400 \mathrm{MHz}, \mathrm{CDCl}_{3}\right) \delta 7.71(\mathrm{~m}, 1 \mathrm{H}$, aromatic $\mathrm{CH}), 7.35$ $(\mathrm{m}, 12 \mathrm{H}$, aromatic $\mathrm{CH}), 5.83(\mathrm{t}, J=7.4 \mathrm{~Hz}, 1 \mathrm{H}, \mathrm{C}=\mathrm{CH}), 3.05(\mathrm{t}, J=7.4 \mathrm{~Hz}, 2 \mathrm{H}$, PhCOCH$\left.H_{2}\right), 2.55\left(\mathrm{~m}, 5 \mathrm{H}, \mathrm{C}=\mathrm{CHCH}_{2}\right.$ and $\left.\mathrm{CH}_{3}\right), 2.18\left(\mathrm{~s}, 3 \mathrm{H}, \mathrm{CH}_{3}\right), 2.04(\mathrm{~m}, 2 \mathrm{H}$, $\left.\mathrm{C}=\mathrm{CHCH}_{2} \mathrm{CH}_{2}\right) .{ }^{13} \mathrm{C}\left\{{ }^{1} \mathrm{H}\right\} \mathrm{NMR}\left(100 \mathrm{MHz}, \mathrm{CDCl}_{3}\right) \delta 204.4(\mathrm{Cq}, \mathrm{C}=\mathrm{O}), 143.9,142.5$, 140.1, 138.2, 138.0, and 136.1 (Cq), 132.0, 131.3, 131.2, 130.3, 130.2, 129.4, 128.4, 128.0, 127.2, 126.8, 125.7, and 125.6 (aromatic $\mathrm{CH}), 41.1\left(\mathrm{PhCOCH}_{2}\right), 28.9$ $\left(\mathrm{C}=\mathrm{CHCH}_{2}\right), 24.9\left(\mathrm{C}=\mathrm{CHCH}_{2} \mathrm{CH}_{2}\right), 21.3\left(\mathrm{CH}_{3}\right), 20.6\left(\mathrm{CH}_{3}\right)$. HRMS Calcd for $\mathrm{C}_{26} \mathrm{H}_{26} \mathrm{O}[\mathrm{M}+\mathrm{H}]^{+}:$355.2062, found: 355.2060 .

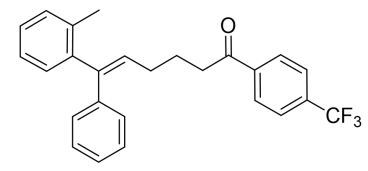

(E)-6-Phenyl-6-(o-tolyl)-1-(4-(trifluoromethyl)phenyl)hex-5-en-1-one

(5d):

$108 \mathrm{mg}, 89 \%$ yield, yellow solid, m.p.: $71-73{ }^{\circ} \mathrm{C} .{ }^{1} \mathrm{H}$ NMR $\left(400 \mathrm{MHz}, \mathrm{CDCl}_{3}\right) \delta 7.97$ $(\mathrm{d}, J=8.1 \mathrm{~Hz}, 2 \mathrm{H}$, aromatic $\mathrm{CH}), 7.67(\mathrm{~d}, J=8.2 \mathrm{~Hz}, 2 \mathrm{H}$, aromatic $\mathrm{CH}), 7.14(\mathrm{~m}, 9$ $\mathrm{H}$, aromatic $\mathrm{CH}), 5.67(\mathrm{t}, J=7.4 \mathrm{~Hz}, 1 \mathrm{H}, \mathrm{C}=\mathrm{CH}), 2.97(\mathrm{t}, J=7.3 \mathrm{~Hz}, 2 \mathrm{H}$, $\left.\mathrm{PhCOCH}_{2}\right), 2.41\left(\mathrm{dd}, J=14.9\right.$ and $\left.7.4 \mathrm{~Hz}, 2 \mathrm{H}, \mathrm{C}=\mathrm{CHCH}_{2}\right), 2.01(\mathrm{~s}, 3 \mathrm{H}, \mathrm{CH}), 1.91$ $\left(\mathrm{m}, 2 \mathrm{H}, \mathrm{C}=\mathrm{CHCH}_{2} \mathrm{CH}_{2}\right) .{ }^{13} \mathrm{C}\left\{{ }^{1} \mathrm{H}\right\} \mathrm{NMR}\left(100 \mathrm{MHz}, \mathrm{CDCl}_{3}\right) \delta 199.1(\mathrm{Cq}, \mathrm{C}=\mathrm{O})$, 143.8, 142.8, 140.1, 139.7, and $136.1(\mathrm{Cq}), 134.35$ (q, $J=32.5 \mathrm{~Hz}, \mathrm{Cq}), 131.0,130.4$, 130.2, 129.4, 128.4, 128.1, 127.3, and 126.9 (aromatic $\mathrm{CH}$ ), 125.7 (q, $J=3.6 \mathrm{~Hz}$, aromatic $\mathrm{CH}$ ), 125.7 (aromatic $\mathrm{CH}) 123.7$ (q, $\left.J=272.7 \mathrm{~Hz}, \mathrm{CF}_{3}\right), 38.4\left(\mathrm{PhCOCH}_{2}\right)$, $28.8\left(\mathrm{C}=\mathrm{CHCH}_{2}\right), 24.5\left(\mathrm{C}=\mathrm{CHCH}_{2} \mathrm{CH}_{2}\right), 20.6\left(\mathrm{CH}_{3}\right)$. HRMS Calcd for $\mathrm{C}_{26} \mathrm{H}_{23} \mathrm{~F}_{3} \mathrm{O}$ $[\mathrm{M}+\mathrm{H}]^{+}:$409.1779, found: 409.1781 .

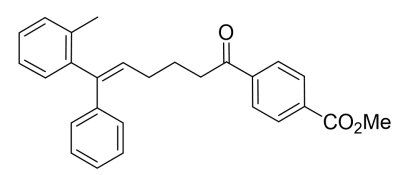

Methyl (E)-4-(6-phenyl-6-(o-tolyl)hex-5-enoyl)benzoate (5e): $101 \mathrm{mg}, 85 \%$ yield, pale yellow solid, m.p.: 96-98 ${ }^{\circ} \mathrm{C} .{ }^{1} \mathrm{H}$ NMR $\left(400 \mathrm{MHz}, \mathrm{CDCl}_{3}\right) \delta 8.13(\mathrm{~d}, J=$ 
$8.4 \mathrm{~Hz}, 2 \mathrm{H}$, aromatic $\mathrm{CH}), 7.98(\mathrm{~d}, J=8.4 \mathrm{~Hz}, 2 \mathrm{H}$, aromatic $\mathrm{CH}), 7.21(\mathrm{~m}, 9 \mathrm{H}$, aromatic $\mathrm{CH}), 5.73(\mathrm{t}, J=7.4 \mathrm{~Hz}, 1 \mathrm{H}, \mathrm{C}=\mathrm{CH}), 3.96\left(\mathrm{~s}, 3 \mathrm{H}, \mathrm{COOCH}_{3}\right), 3.03(\mathrm{t}, J=$ $\left.7.3 \mathrm{~Hz}, 2 \mathrm{H}, \mathrm{PhCOCH}_{2}\right), 2.46\left(\mathrm{dd}, J=14.9\right.$ and $\left.7.4 \mathrm{~Hz}, 2 \mathrm{H}, \mathrm{C}=\mathrm{CHCH}_{2}\right), 2.07$ (s, $3 \mathrm{H}$, $\left.\mathrm{CH}_{3}\right), 1.96\left(\mathrm{~m}, 2 \mathrm{H}, \mathrm{C}=\mathrm{CHCH}_{2} \mathrm{CH}_{2}\right) .{ }^{13} \mathrm{C}\left\{{ }^{1} \mathrm{H}\right\} \mathrm{NMR}\left(100 \mathrm{MHz}, \mathrm{CDCl}_{3}\right) \delta 199.5(\mathrm{Cq}$, $\mathrm{C}=\mathrm{O}), 166.2\left(\mathrm{Cq}, \mathrm{CO}_{2} \mathrm{CH}_{3}\right), 143.8,142.6,140.2,140.1,136.0$, and $133.8(\mathrm{Cq}), 131.0$, $130.3,130.1,129.8,129.3,128.0,128.0,127.2,126.8$, and 125.6 (aromatic CH), 52.5 $\left(\mathrm{CO}_{2} \mathrm{CH}_{3}\right), 38.4\left(\mathrm{PhCOCH}_{2}\right), 28.8\left(\mathrm{C}=\mathrm{CHCH}_{2}\right), 24.5\left(\mathrm{C}=\mathrm{CHCH}_{2} \mathrm{CH}_{2}\right), 20.5\left(\mathrm{CH}_{3}\right)$. HRMS Calcd for $\mathrm{C}_{27} \mathrm{H}_{26} \mathrm{O}_{3}[\mathrm{M}+\mathrm{Na}]^{+}:$421.1780, found: 421.1780 .

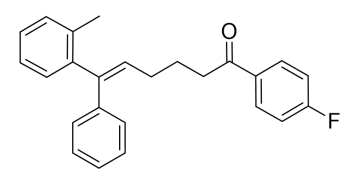

(E)-1-(4-Fluorophenyl)-6-phenyl-6-(o-tolyl)hex-5-en-1-one (5f): $96 \mathrm{mg}$, 90\% yield, pale yellow solid, m.p.: 63-65 ${ }^{\circ} \mathrm{C} .{ }^{1} \mathrm{H}$ NMR $\left(400 \mathrm{MHz}, \mathrm{CDCl}_{3}\right) \delta 7.91(\mathrm{~m}, 2 \mathrm{H}$, aromatic $\mathrm{CH}), 7.14(\mathrm{~m}, 11 \mathrm{H}$, aromatic $\mathrm{CH}), 5.67$ (t, $J=7.4 \mathrm{~Hz}, 1 \mathrm{H}, \mathrm{C}=\mathrm{CH}), 2.92$ (t, $\left.J=7.4 \mathrm{~Hz}, 2 \mathrm{H}, \mathrm{PhCOCH}_{2}\right), 2.39\left(\mathrm{dd}, J=14.9\right.$ and $\left.7.4 \mathrm{~Hz}, 2 \mathrm{H}, \mathrm{C}=\mathrm{CHCH}_{2}\right), 2.01(\mathrm{~s}$, $\left.3 \mathrm{H}, \mathrm{CH}_{3}\right), 1.89\left(\mathrm{~m}, 2 \mathrm{H}, \mathrm{C}=\mathrm{CHCH}_{2} \mathrm{CH}_{2}\right) \cdot{ }^{13} \mathrm{C}\left\{{ }^{1} \mathrm{H}\right\} \mathrm{NMR}\left(100 \mathrm{MHz}, \mathrm{CDCl}_{3}\right) \delta 198.5$ $(\mathrm{Cq}, \mathrm{C}=\mathrm{O}), 165.76(\mathrm{~d}, J=254.4 \mathrm{~Hz}, \mathrm{Cq}), 143.9,142.6,140.2$, and $136.1(\mathrm{Cq}), 133.5$ (d, $J=3.0 \mathrm{~Hz}, \mathrm{Cq}), 131.2$ (aromatic $\mathrm{CH}), 130.7$ (d, $J=9.3 \mathrm{~Hz}$, aromatic $\mathrm{CH}), 130.3$, 130.2, 129.4, 128.1, 127.2, 126.8, and 125.7 (aromatic $\mathrm{CH}$ ), 115.7 (d, $J=21.8 \mathrm{~Hz}$, aromatic $\mathrm{CH}), 38.1\left(\mathrm{PhCOCH}_{2}\right), 28.9\left(\mathrm{C}=\mathrm{CHCH}_{2}\right), 24.7\left(\mathrm{C}=\mathrm{CHCH}_{2} \mathrm{CH}_{2}\right), 20.6(\mathrm{~s}$ $\mathrm{CH}_{3}$ ). HRMS Calcd for $\mathrm{C}_{25} \mathrm{H}_{23} \mathrm{FO}[\mathrm{M}+\mathrm{H}]^{+}: 359.1811$, found: 359.1812 .

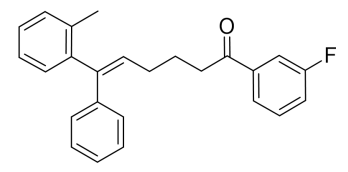

(E)-1-(3-Fluorophenyl)-6-phenyl-6-(o-tolyl)hex-5-en-1-one (5g): 93 mg, 87\% yield, yellow solid, m.p.: $81-83{ }^{\circ} \mathrm{C} .{ }^{1} \mathrm{H}$ NMR $\left(400 \mathrm{MHz}, \mathrm{CDCl}_{3}\right) \delta 7.66(\mathrm{~d}, J=7.8 \mathrm{~Hz}$, $1 \mathrm{H}$, aromatic $\mathrm{CH}), 7.56(\mathrm{~m}, 1 \mathrm{H}$, aromatic $\mathrm{CH}), 7.37(\mathrm{~m}, 1 \mathrm{H}$, aromatic $\mathrm{CH}), 7.15(\mathrm{~m}$, $10 \mathrm{H}$, aromatic $\mathrm{CH}), 5.67(\mathrm{t}, J=7.4 \mathrm{~Hz}, 1 \mathrm{H}, \mathrm{C}=\mathrm{CH}), 2.92(\mathrm{t}, J=7.4 \mathrm{~Hz}, 2 \mathrm{H}$, $\mathrm{PhCOCH}_{2}$ ), 2.39 (dd, $J=14.9$ and $\left.7.4 \mathrm{~Hz}, 2 \mathrm{H}, \mathrm{C}=\mathrm{CHCH}_{2}\right), 2.01\left(\mathrm{~s}, 3 \mathrm{H}, \mathrm{CH}_{3}\right), 1.89$ $\left(\mathrm{m}, 2 \mathrm{H}, \mathrm{C}=\mathrm{CHCH}_{2} \mathrm{CH}_{2}\right) .{ }^{13} \mathrm{C}\left\{{ }^{1} \mathrm{H}\right\} \mathrm{NMR}\left(100 \mathrm{MHz}, \mathrm{CDCl}_{3}\right) \delta 198.7(\mathrm{~d}, J=2.0 \mathrm{~Hz}$, $\mathrm{Cq}, \mathrm{C}=\mathrm{O}), 162.9(\mathrm{~d}, \mathrm{~J}=247.9 \mathrm{~Hz}, \mathrm{Cq}), 143.9,142.7$, and $140.1(\mathrm{Cq}), 139.2(\mathrm{~d}, J=6.0$ $\mathrm{Hz}, \mathrm{Cq}), 136.1(\mathrm{Cq}), 131.1$ (aromatic CH), 130.3 (d, J = 7.6 Hz, aromatic CH), 130.3, 
130.2. 129.4, 128.0, 127.2, 126.8, and 125.6 (aromatic $\mathrm{CH}), 123.8(\mathrm{~d}, J=3.0 \mathrm{~Hz}$, aromatic $\mathrm{CH}), 120.0(\mathrm{~d}, J=21.5 \mathrm{~Hz}$, aromatic $\mathrm{CH}), 114.8(\mathrm{~d}, J=22.2 \mathrm{~Hz}$, aromatic $\mathrm{CH}), 38.3\left(\mathrm{PhCOCH}_{2}\right), 28.8\left(\mathrm{C}=\mathrm{CHCH}_{2}\right), 24.5\left(\mathrm{C}=\mathrm{CHCH}_{2} \mathrm{CH}_{2}\right), 20.5\left(\mathrm{CH}_{3}\right)$. HRMS Calcd for $\mathrm{C}_{25} \mathrm{H}_{23} \mathrm{FO}[\mathrm{M}+\mathrm{H}]^{+}$: 359.1811; Found: 359.1806 .

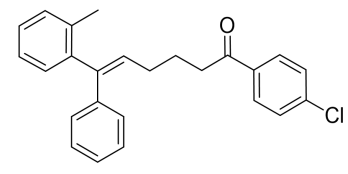

(E)-1-(4-Chlorophenyl)-6-phenyl-6-(o-tolyl)hex-5-en-1-one (5h): $97 \mathrm{mg}, 87 \%$ yield, yellow solid, m.p.: $87-89{ }^{\circ} \mathrm{C} .{ }^{1} \mathrm{H}$ NMR $\left(400 \mathrm{MHz}, \mathrm{CDCl}_{3}\right) \delta 7.81(\mathrm{~m}, 2 \mathrm{H}$, aromatic $\mathrm{CH}), 7.37(\mathrm{~m}, 2 \mathrm{H}$, aromatic $\mathrm{CH}), 7.16(\mathrm{~m}, 9 \mathrm{H}$, aromatic $\mathrm{CH}), 5.66(\mathrm{t}, J=$ $7.4 \mathrm{~Hz}, 1 \mathrm{H}, \mathrm{C}=\mathrm{CH}), 2.92\left(\mathrm{t}, J=7.4 \mathrm{~Hz}, 2 \mathrm{H}, \mathrm{PhCOCH} H_{2}\right), 2.39(\mathrm{dd}, J=14.9$ and 7.4 $\left.\mathrm{Hz}, 2 \mathrm{H}, \mathrm{C}=\mathrm{CHCH}_{2}\right), 2.00\left(\mathrm{~s}, 3 \mathrm{H}, \mathrm{CH}_{3}\right), 1.88\left(\mathrm{~m}, 2 \mathrm{H}, \mathrm{C}=\mathrm{CHCH}_{2} \mathrm{CH}_{2}\right) .{ }^{13} \mathrm{C}\left\{{ }^{1} \mathrm{H}\right\}$ NMR $\left(100 \mathrm{MHz}, \mathrm{CDCl}_{3}\right) \delta 198.9(\mathrm{Cq}, \mathrm{C}=\mathrm{O}), 143.9,142.7,140.1,139.5,136.1$, and $135.4(\mathrm{Cq}), 131.1,130.3,130.2,129.6,129.4,129.0,128.1,127.2,126.8$, and 125.7 (aromatic $\mathrm{CH}), 38.1\left(\mathrm{PhCOCH}_{2}\right), 28.9\left(\mathrm{C}=\mathrm{CHCH}_{2}\right), 24.6\left(\mathrm{C}=\mathrm{CHCH}_{2} \mathrm{CH}_{2}\right), 20.6$ $\left(\mathrm{CH}_{3}\right)$. HRMS Calcd for $\mathrm{C}_{25} \mathrm{H}_{23} \mathrm{ClO}[\mathrm{M}+\mathrm{H}]^{+}: 375.1516$, found: 375.1518 .

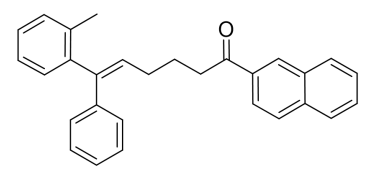

(E)-1-(Naphthalen-2-yl)-6-phenyl-6-(o-tolyl)hex-5-en-1-one $\quad(5 i): 100$ mg, $85 \%$ yield, pale yellow solid, m.p.: $124-126^{\circ} \mathrm{C} .{ }^{1} \mathrm{H}$ NMR $\left(400 \mathrm{MHz}, \mathrm{CDCl}_{3}\right) \delta 8.39(\mathrm{~s}$, $1 \mathrm{H}$, aromatic $\mathrm{CH}), 7.90(\mathrm{~m}, 4 \mathrm{H}$, aromatic $\mathrm{CH}), 7.53(\mathrm{~m}, 2 \mathrm{H}$, aromatic $\mathrm{CH}), 7.16(\mathrm{~m}$, $9 \mathrm{H}$, aromatic $\mathrm{CH}), 5.71(\mathrm{t}, J=7.4 \mathrm{~Hz}, 1 \mathrm{H}, \mathrm{C}=\mathrm{CH}), 3.08(\mathrm{t}, J=7.4 \mathrm{~Hz}, 2 \mathrm{H}$, PhCOCH$\left.H_{2}\right), 2.44\left(\mathrm{dd}, J=14.9\right.$ and $\left.7.4 \mathrm{~Hz}, 2 \mathrm{H}, \mathrm{C}=\mathrm{CHCH}_{2}\right), 1.97\left(\mathrm{~m}, 5 \mathrm{H}, \mathrm{CH}_{3}\right.$ and $\left.\mathrm{C}=\mathrm{CHCH}_{2} \mathrm{CH}_{2}\right) .{ }^{13} \mathrm{C}\left\{{ }^{1} \mathrm{H}\right\} \mathrm{NMR}\left(100 \mathrm{MHz}, \mathrm{CDCl}_{3}\right) \delta 200.1(\mathrm{Cq}, \mathrm{C}=\mathrm{O}), 143.9,142.5$, 140.2, 136.2, 135.6, 134.4, and 132.6 (Cq) 131.3, 130.3, 130.3, 129.7, 129.6, 129.41, $128.5,128.5,128.1,127.9,127.22,126.8,126.8,125.7$, and 124.1 (aromatic $\mathrm{CH}$ ), $38.2\left(\mathrm{PhCOCH}_{2}\right), 29.0\left(\mathrm{C}=\mathrm{CHCH}_{2}\right), 24.9\left(\mathrm{C}=\mathrm{CHCH}_{2} \mathrm{CH}_{2}\right), 20.6\left(\mathrm{CH}_{3}\right)$. HRMS Calcd for $\mathrm{C}_{29} \mathrm{H}_{26} \mathrm{O}[\mathrm{M}+\mathrm{H}]^{+}:$391.2062, found: 391.2060.

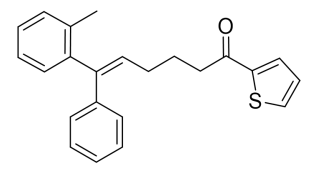

(E)-6-Phenyl-1-(thiophen-2-yl)-6-(o-tolyl)hex-5-en-1-one (5j): 93 mg, 89\% 
yield, pale yellow solid, m.p.: $103-105{ }^{\circ} \mathrm{C} .{ }^{1} \mathrm{H}$ NMR $\left(400 \mathrm{MHz}, \mathrm{CDCl}_{3}\right) \delta 7.76(\mathrm{~m}, 2$ $\mathrm{H}$, aromatic $\mathrm{CH}), 7.31(\mathrm{~m}, 10 \mathrm{H}$, aromatic $\mathrm{CH}), 5.84(\mathrm{t}, J=7.4 \mathrm{~Hz}, 1 \mathrm{H}, \mathrm{C}=\mathrm{CH}), 3.06$ (t, $\left.J=7.5 \mathrm{~Hz}, 2 \mathrm{H}, \mathrm{PhCOCH}_{2}\right), 2.56\left(\mathrm{dd}, J=14.9\right.$ and $\left.7.4 \mathrm{~Hz}, 2 \mathrm{H}, \mathrm{C}=\mathrm{CHCH}_{2}\right), 2.18$ $\left(\mathrm{s}, 3 \mathrm{H}, \mathrm{CH}_{3}\right), 2.08\left(\mathrm{~m}, 2 \mathrm{H}, \mathrm{C}=\mathrm{CHCH}_{2} \mathrm{CH}_{2}\right) .{ }^{13} \mathrm{C}\left\{{ }^{1} \mathrm{H}\right\} \mathrm{NMR}\left(100 \mathrm{MHz}, \mathrm{CDCl}_{3}\right) \delta$ $192.9(\mathrm{Cq}, \mathrm{C}=\mathrm{O}), 144.4,143.8,142.5,140.1$, and $136.0(\mathrm{Cq}), 133.4,131.7,131.1$, $130.2,130.1,129.3,128.1,128.0,127.2,126.7$, and 125.6 (aromatic $\mathrm{CH}$ ), 38.8 $\left(\mathrm{PhCOCH}_{2}\right), 28.8\left(\mathrm{C}=\mathrm{CHCH}_{2}\right), 25.0\left(\mathrm{C}=\mathrm{CHCH}_{2} \mathrm{CH}_{2}\right), 20.5\left(\mathrm{CH}_{3}\right)$. HRMS Calcd for $\mathrm{C}_{23} \mathrm{H}_{22} \mathrm{OS}[\mathrm{M}+\mathrm{H}]^{+}: 347.1470$, found: 347.1473 .

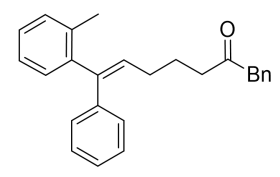

(E)-1,7-Diphenyl-7-(o-tolyl)hept-6-en-2-one (5k): $94 \mathrm{mg}, 89 \%$ yield, pale yellow liquid. ${ }^{1} \mathrm{H}$ NMR (400 MHz, $\left.\mathrm{CDCl}_{3}\right) \delta 7.17(\mathrm{~m}, 14 \mathrm{H}$, aromatic $\mathrm{CH}), 5.54(\mathrm{t}, J$ $=7.4 \mathrm{~Hz}, 1 \mathrm{H}, \mathrm{C}=\mathrm{CH}), 3.58\left(\mathrm{~s}, 2 \mathrm{H}, \mathrm{PhCH}_{2}\right), 2.41\left(\mathrm{t}, J=7.4 \mathrm{~Hz}, 2 \mathrm{H}, \mathrm{C}=\mathrm{CHCH}_{2}\right)$, $2.23\left(\mathrm{dd}, J=14.8\right.$ and $\left.7.4 \mathrm{~Hz}, 2 \mathrm{H}, \mathrm{C}=\mathrm{CHCH}_{2}\right), 1.97\left(\mathrm{~s}, 3 \mathrm{H}, \mathrm{CH}_{3}\right), 1.68(\mathrm{~m}, 2 \mathrm{H}$, $\left.\mathrm{C}=\mathrm{CHCH}_{2} \mathrm{CH}_{2}\right) .{ }^{13} \mathrm{C}\left\{{ }^{1} \mathrm{H}\right\} \mathrm{NMR}\left(100 \mathrm{MHz}, \mathrm{CDCl}_{3}\right) \delta 208.2(\mathrm{Cq}, \mathrm{C}=\mathrm{O}), 143.8,142.4$, 140.1, 136.0, and 134.4 (Cq), 131.1, 130.3, 130.2, 129.5, 129.3, 128.8, 128.0, 127.2, 127.0, 126.7, and 125.6 (aromatic $\mathrm{CH}), 50.3\left(\mathrm{PhCH}_{2} \mathrm{CO}\right), 41.3\left(\mathrm{BnCOCH}_{2}\right), 28.6$ $\left(\mathrm{C}=\mathrm{CHCH}_{2}\right), 24.1\left(\mathrm{C}=\mathrm{CHCH}_{2} \mathrm{CH}_{2}\right), 20.5\left(\mathrm{CH}_{3}\right)$. HRMS Calcd for $\mathrm{C}_{26} \mathrm{H}_{26} \mathrm{O}[\mathrm{M}+\mathrm{H}]^{+}$: 355.2062, found: 355.2058 .

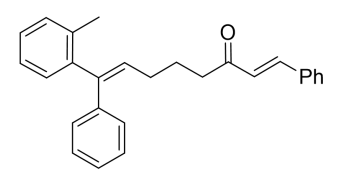

(1E,7E)-1,8-Diphenyl-8-(o-tolyl)octa-1,7-dien-3-one (5l): $103 \mathrm{mg}, 94 \%$ yield, pale yellow solid, m.p.: 106-108 ${ }^{\circ} \mathrm{C} .{ }^{1} \mathrm{H}$ NMR (400 MHz, $\left.\mathrm{CDCl}_{3}\right) \delta 7.67$ (m, $3 \mathrm{H}$, aromatic $\mathrm{CH}), 7.52(\mathrm{~m}, 3 \mathrm{H}$, aromatic $\mathrm{CH}), 7.34(\mathrm{~m}, 9 \mathrm{H}$, aromatic $\mathrm{CH}$ and $\mathrm{CH}=\mathrm{CH})$, $6.86(\mathrm{~d}, J=16.2 \mathrm{~Hz}, 1 \mathrm{H}, \mathrm{CH}=\mathrm{CH}), 5.84(\mathrm{t}, J=7.3 \mathrm{~Hz}, 1 \mathrm{H}, \mathrm{C}=\mathrm{CH}), 2.83(\mathrm{t}, J=7.4$ $\left.\mathrm{Hz}, 2 \mathrm{H}, \mathrm{C}=\mathrm{CHCH}_{2}\right), 2.55\left(\mathrm{dd}, J=14.8\right.$ and $\left.7.4 \mathrm{~Hz}, 2 \mathrm{H}, \mathrm{C}=\mathrm{CHCH}_{2}\right), 2.20(\mathrm{~s}, 3 \mathrm{H}$, $\left.\mathrm{CH}_{3}\right), 2.02\left(\mathrm{~m}, 2 \mathrm{H}, \mathrm{C}=\mathrm{CHCH}_{2} \mathrm{CH}_{2}\right) .{ }^{13} \mathrm{C}\left\{{ }^{1} \mathrm{H}\right\} \mathrm{NMR}\left(100 \mathrm{MHz}, \mathrm{CDCl}_{3}\right) \delta 200.1(\mathrm{Cq}$, $\mathrm{C}=\mathrm{O}), 143.9,142.4,140.1,136.1,134.6,131.3,130.5,130.3,130.2,129.3,129.0$, $128.3,128.0,127.2,126.7,126.3,125.6,40.4,28.9,24.7,20.5$. HRMS Calcd for $\mathrm{C}_{27} \mathrm{H}_{26} \mathrm{O}[\mathrm{M}+\mathrm{H}]^{+}:$367.2062, found: 367.2063 . 


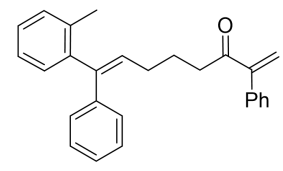

(E)-2,8-Diphenyl-8-(o-tolyl)octa-1,7-dien-3-one (5m): $61 \mathrm{mg}, 55 \%$ yield, colorless liquid. ${ }^{1} \mathrm{H}$ NMR $\left(400 \mathrm{MHz}, \mathrm{CDCl}_{3}\right) \delta 7.20(\mathrm{~m}, 14 \mathrm{H}$, aromatic $\mathrm{CH}), 6.02$ and 5.80 (s each, 1:1 H, C=CH2), $5.61(\mathrm{t}, J=7.4 \mathrm{~Hz}, 1 \mathrm{H}, \mathrm{C}=\mathrm{CH}), 2.72(\mathrm{t}, J=7.4 \mathrm{~Hz}, 2 \mathrm{H}$, $\left.\mathrm{C}=\mathrm{CHCH}_{2}\right), 2.33\left(\mathrm{dd}, J=14.8\right.$ and $\left.7.4 \mathrm{~Hz}, 2 \mathrm{H}, \mathrm{C}=\mathrm{CHCH}_{2}\right), 1.99\left(\mathrm{~s}, 3 \mathrm{H}, \mathrm{CH}_{3}\right), 1.81$ $\left(\mathrm{m}, 2 \mathrm{H}, \mathrm{C}=\mathrm{CHCH}_{2} \mathrm{CH}_{2}\right) .{ }^{13} \mathrm{C}\left\{{ }^{1} \mathrm{H}\right\} \mathrm{NMR}\left(100 \mathrm{MHz}, \mathrm{CDCl}_{3}\right) \delta 202.0(\mathrm{Cq}, \mathrm{C}=\mathrm{O})$, 149.6, 143.9, 142.5, 140.2, 137.4, and 136.1 (Cq), 131.3, 130.3, 130.2, 129.4, 128.5, 128.4, 128.2, 128.1, 127.2, 126.8, 125.7, and 124.3 (aromatic $\mathrm{CH}), 39.3\left(\mathrm{PhCOCH}_{2}\right)$, $28.8\left(\mathrm{C}=\mathrm{CHCH}_{2}\right), 24.8\left(\mathrm{C}=\mathrm{CHCH}_{2} \mathrm{CH}_{2}\right), 20.6\left(\mathrm{CH}_{3}\right)$. HRMS Calcd for $\mathrm{C}_{27} \mathrm{H}_{26} \mathrm{O}$ $[\mathrm{M}+\mathrm{H}]^{+}:$367.2062, found: 367.2065.

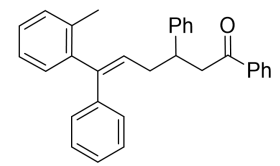

(E)-1,3,6-Triphenyl-6-(o-tolyl)hex-5-en-1-one (5n): $117 \mathrm{mg}, 94 \%$ yield, pale yellow liquid. ${ }^{1} \mathrm{H}$ NMR (400 MHz, $\left.\mathrm{CDCl}_{3}\right) \delta 7.85(\mathrm{~d}, J=7.3 \mathrm{~Hz}, 2 \mathrm{H}$, aromatic $\mathrm{CH})$, $7.49(\mathrm{t}, J=7.4 \mathrm{~Hz}, 1 \mathrm{H}$, aromatic $\mathrm{CH}), 7.38(\mathrm{t}, J=7.6 \mathrm{~Hz}, 2 \mathrm{H}$, aromatic $\mathrm{CH}), 7.12(\mathrm{~m}$, $13 \mathrm{H}$, aromatic $\mathrm{CH}), 6.94(\mathrm{~m}, 1 \mathrm{H}$, aromatic $\mathrm{CH}), 5.56(\mathrm{t}, J=7.2 \mathrm{~Hz}, 1 \mathrm{H}, \mathrm{C}=\mathrm{CH})$, $3.56\left(\mathrm{~m}, 1 \mathrm{H}, \mathrm{PhCOCH}_{2}\right), 3.27\left(\mathrm{~m}, 1: 1 \mathrm{H}, \mathrm{PhCH}\right.$ and $\left.\mathrm{PhCOCH}_{2}\right), 2.72(\mathrm{~m}, 2 \mathrm{H}$, $\left.\mathrm{C}=\mathrm{CHCH}_{2}\right), 1.89\left(\mathrm{~s}, 3 \mathrm{H}, \mathrm{CH}_{3}\right) .{ }^{13} \mathrm{C}\left\{{ }^{1} \mathrm{H}\right\} \mathrm{NMR}\left(100 \mathrm{MHz}, \mathrm{CDCl}_{3}\right) \delta 198.8(\mathrm{Cq}, \mathrm{C}=\mathrm{O})$, 144.2, 143.8, 143.1, 140.1, 137.2, and 136.1 (Cq), 133.0, 130.2, 130.1, 129.6, 129.4, 128.6, 128.5, 128.1, 128.0, 127.8, 127.1, 126.7, 126.5, and 125.5 (aromatic CH), 45.2 $\left(\mathrm{PhCOCH}_{2}\right), 41.8\left(\mathrm{C}=\mathrm{CHCH}_{2}\right), 35.8(\mathrm{PhCH}), 20.4\left(\mathrm{CH}_{3}\right)$. HRMS Calcd for $\mathrm{C}_{31} \mathrm{H}_{28} \mathrm{O}$ $[\mathrm{M}+\mathrm{H}]^{+}:$417.2218, found: 417.2221.

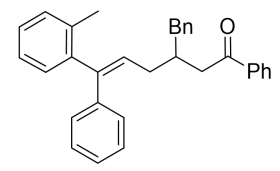

(E)-3-Benzyl-1,6-diphenyl-6-(o-tolyl)hex-5-en-1-one (5o): $122 \mathrm{mg}, 94 \%$ yield, yellow liquid. ${ }^{1} \mathrm{H}$ NMR (400 MHz, $\left.\mathrm{CDCl}_{3}\right) \delta 7.89$ (d, $J=7.7 \mathrm{~Hz}, 2 \mathrm{H}$, aromatic $\left.\mathrm{CH}\right)$, $7.58(\mathrm{~m}, 1 \mathrm{H}$, aromatic $\mathrm{CH}), 7.46(\mathrm{t}, J=7.1 \mathrm{~Hz}, 2 \mathrm{H}$, aromatic $\mathrm{CH}), 7.35-7.26(\mathrm{~m}, 5$ $\mathrm{H}$, aromatic $\mathrm{CH}), 7.26-7.21(\mathrm{~m}, 5 \mathrm{H}$, aromatic $\mathrm{CH}), 7.17$ (m, $4 \mathrm{H}$, aromatic $\mathrm{CH}), 5.82$ (t, $J=6.7 \mathrm{~Hz}, 1 \mathrm{H}$, alkenyl CH), $2.96\left(\mathrm{~m}, 2 \mathrm{H}, \mathrm{CH}_{2} \mathrm{CO}\right), 2.81\left(\mathrm{~m}, 1 \mathrm{H}, \mathrm{CHCH}_{2}\right), 2.71$ 
(m, $\left.2 \mathrm{H} \mathrm{CH}_{2}\right), 2.50\left(\mathrm{~m}, 2 \mathrm{H}, \mathrm{CH}_{2}\right), 2.08\left(\mathrm{~s}, 3 \mathrm{H}, \mathrm{CH}_{3}\right) .{ }^{13} \mathrm{C}\left\{{ }^{1} \mathrm{H}\right\}$ NMR (100 MHz, $\left.\mathrm{CDCl}_{3}\right) \delta 199.78(\mathrm{Cq}, \mathrm{C}=\mathrm{O}), 143.9,143.6,140.2,140.0,137.2$, and $136.0(\mathrm{Cq}), 132.9$, $130.3,130.1,129.8,129.4,129.3,128.6,128.4,128.1,128.0,127.2,126.7,126.1$, and 125.6 (aromatic $\mathrm{CH}$ and alkenyl $\mathrm{CH}), 42.3,40.3,37.3,33.0$, and $20.6\left(\mathrm{CH}, \mathrm{CH}_{2}\right.$ and $\mathrm{CH}_{3}$ ). HRMS Calcd for $\mathrm{C}_{32} \mathrm{H}_{30} \mathrm{O}[\mathrm{M}+\mathrm{H}]^{+}:$431.2375, found: 431.2374 .

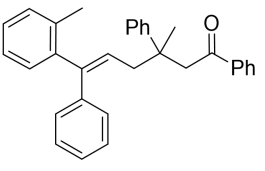

(E)-3-Methyl-1,3,6-triphenyl-6-(o-tolyl)hex-5-en-1-one (5 p): $119 \quad \mathrm{mg}, 92 \%$ yield, white solid, m.p.: 102-104 ${ }^{\circ} \mathrm{C} .{ }^{1} \mathrm{H}$ NMR $\left(400 \mathrm{MHz}, \mathrm{CDCl}_{3}\right) \delta 7.81(\mathrm{~m}, 2 \mathrm{H}$, aromatic $\mathrm{CH}), 7.52(\mathrm{t}, J=7.4 \mathrm{~Hz}, 1 \mathrm{H}$, aromatic $\mathrm{CH}), 7.33(\mathrm{~m}, 9 \mathrm{H}$, aromatic $\mathrm{CH})$, $7.15(\mathrm{~m}, 6 \mathrm{H}$, aromatic $\mathrm{CH}), 6.98(\mathrm{~m}, 1 \mathrm{H}$, aromatic $\mathrm{CH}), 5.54(\mathrm{t}, J=7.2 \mathrm{~Hz}, 1 \mathrm{H}$, $\mathrm{C}=\mathrm{CH}$ ), 3.50 and $3.26\left(\mathrm{~d}\right.$ each, $\left.J=16.0 \mathrm{~Hz}, 1: 1 \mathrm{H}, \mathrm{C}=\mathrm{CHCH}_{2}\right), 2.95(\mathrm{~m}, 2 \mathrm{H}$, $\left.\mathrm{PhCOCH}_{2}\right), 1.97\left(\mathrm{~s}, 3 \mathrm{H}, \mathrm{CH}_{3}\right), 1.64\left(\mathrm{~s}, 3 \mathrm{H}, \mathrm{CH}_{3}\right) .{ }^{13} \mathrm{C}\left\{{ }^{1} \mathrm{H}\right\} \mathrm{NMR}\left(100 \mathrm{MHz}, \mathrm{CDCl}_{3}\right)$ $\delta 198.7(\mathrm{Cq}, \mathrm{C}=\mathrm{O}), 146.5,144.1,143.7,140.3,138.2$, and $136.0(\mathrm{Cq}), 132.7,130.2$, $130.1,129.5,128.4,128.3,128.1,128.0,127.9,127.1,126.8,126.3,125.9$, and 125.5 (aromatic $\mathrm{CH}), 50.0\left(\mathrm{PhCOCH}_{2}\right), 42.0\left(\mathrm{C}=\mathrm{CHCH}_{2}\right), 41.5(\mathrm{PhC}), 24.7\left(\mathrm{CH}_{3}\right), 20.5$ $\left(\mathrm{CH}_{3}\right)$. HRMS Calcd for $\mathrm{C}_{32} \mathrm{H}_{30} \mathrm{O}[\mathrm{M}+\mathrm{H}]^{+}:$431.2375, found: 431.2377 .

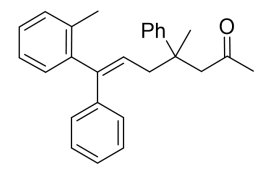

(E)-4-Methyl-4,7-diphenyl-7-(o-tolyl)hept-6-en-2-one (5q): $75 \mathrm{mg}$, 68\% yield, pale yellow liquid. ${ }^{1} \mathrm{H}$ NMR $\left(400 \mathrm{MHz}, \mathrm{CDCl}_{3}\right) \delta 7.18(\mathrm{~m}, 8 \mathrm{H}$, aromatic $\mathrm{CH}), 7.03$ (m, $5 \mathrm{H}$, aromatic $\mathrm{CH}), 6.88(\mathrm{~d}, J=7.0 \mathrm{~Hz}, 1 \mathrm{H}$, aromatic $\mathrm{CH}), 5.38(\mathrm{t}, J=7.1 \mathrm{~Hz}, 1$ $\mathrm{H}, \mathrm{C}=\mathrm{CH}), 2.84\left(\mathrm{~m}, 1: 1 \mathrm{H}, \mathrm{PhCOCH}_{2}\right.$ and $\left.\mathrm{C}=\mathrm{CHCH}_{2}\right), 2.67(\mathrm{dd}, J=14.8$ and $7.4 \mathrm{~Hz}$, $\left.1 \mathrm{H}, \mathrm{C}=\mathrm{CHCH}_{2}\right), 2.56\left(\mathrm{~d}, J=14.5 \mathrm{~Hz}, 1 \mathrm{H}, \mathrm{PhCOCH}_{2}\right), 1.87\left(\mathrm{~s}, 3 \mathrm{H}, \mathrm{CH}_{3}\right), 1.69$ (s, 3 $\left.\mathrm{H}, \mathrm{CH}_{3}\right), 1.44\left(\mathrm{~s}, 3 \mathrm{H}, \mathrm{CH}_{3}\right) .{ }^{13} \mathrm{C}\left\{{ }^{1} \mathrm{H}\right\} \mathrm{NMR}\left(100 \mathrm{MHz}, \mathrm{CDCl}_{3}\right) \delta 207.9(\mathrm{Cq}, \mathrm{C}=\mathrm{O})$, 145.9, 144.0, 143.8, 140.25, and 136.03 (Cq), 130.2, 130.1, 129.5, 128.4, 128.0, 127.6, 127.1, 126.8, 126.4, 126.2, and 125.5 (aromatic $\mathrm{CH}), 56.0\left(\mathrm{PhCOCH}_{2}\right), 42.0$ $\left(\mathrm{C}=\mathrm{CHCH}_{2}\right), 41.3(\mathrm{PhC}), 32.0\left(\mathrm{CH}_{3}\right), 24.2\left(\mathrm{CH}_{3}\right), 20.5\left(\mathrm{CH}_{3}\right)$. HRMS Calcd for $\mathrm{C}_{27} \mathrm{H}_{28} \mathrm{O}[\mathrm{M}+\mathrm{H}]^{+}:$369.2218, found: 369.2221 . 


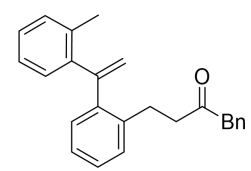

1-phenyl-4-(2-(1-(o-tolyl)vinyl)phenyl)butan-2-one (8a): $44 \mathrm{mg}, 43 \%$; yellow liquid. ${ }^{1} \mathrm{H}$ NMR $\left(400 \mathrm{MHz}, \mathrm{CDCl}_{3}\right) \delta 7.21(\mathrm{~m}, 3 \mathrm{H}$, aromatic $\mathrm{CH}), 7.16(\mathrm{~m}, 1 \mathrm{H}$, aromatic $\mathrm{CH}), 7.11(\mathrm{~m}, 2 \mathrm{H}$, aromatic $\mathrm{CH}), 7.06(\mathrm{~m}, 1 \mathrm{H}$, aromatic $\mathrm{CH}), 7.01(\mathrm{~m}, 5 \mathrm{H}$, aromatic $\mathrm{CH}), 6.95(\mathrm{~m}, 1 \mathrm{H}$, aromatic $\mathrm{CH}), 5.30(\mathrm{~d}, J=1.7 \mathrm{~Hz}, 1 \mathrm{H}$, alkenyl $\mathrm{CH})$, $5.28(\mathrm{~d}, J=1.7 \mathrm{~Hz}, 1 \mathrm{H}$, alkenyl $\mathrm{CH}), 3.36\left(\mathrm{~s}, 2 \mathrm{H}, \mathrm{CH}_{2} \mathrm{Ph}\right), 2.52\left(\mathrm{~m}, 2 \mathrm{H}, \mathrm{CH}_{2} \mathrm{CO}\right)$, $2.18\left(\mathrm{~m}, 2 \mathrm{H}, \mathrm{CH}_{2}\right), 2.03\left(\mathrm{~s}, 3 \mathrm{H}, \mathrm{CH}_{3}\right) .{ }^{13} \mathrm{C} \mathrm{NMR}\left(100 \mathrm{MHz}, \mathrm{CDCl}_{3}\right) \delta 207.3(\mathrm{Cq}$, $\mathrm{C}=\mathrm{O}), 150.2,142.4,141.8,138.6,135.6$, and $134.3(\mathrm{Cq}), 130.9,130.8,129.8,129.7$, $129.5,128.7,127.8,127.5,127.0,126.3$, and 125.91 (aromatic $\mathrm{CH}$ ), 119.7 (alkenyl $\mathrm{CH}), 50.0\left(\mathrm{CH}_{2} \mathrm{Ph}\right), 42.9\left(\mathrm{CH}_{2} \mathrm{CO}\right), 27.6\left(\mathrm{CH}_{2} \mathrm{CH}_{2} \mathrm{CO}\right), 21.0\left(\mathrm{CH}_{3}\right)$. HRMS Calcd for $\mathrm{C}_{25} \mathrm{H}_{24} \mathrm{O},[\mathrm{M}+\mathrm{H}]^{+}:$341.1905; Found: 341.1903 .

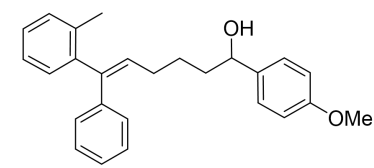

(E)-1-(4-Methoxyphenyl)-6-phenyl-6-(o-tolyl)hex-5-en-1-ol (9): 105 mg, 94\%; colorless liquid. ${ }^{1} \mathrm{H}$ NMR $\left(400 \mathrm{MHz}, \mathrm{CDCl}_{3}\right) \delta 7.14(\mathrm{~m}, 11 \mathrm{H}$, aromatic $\mathrm{CH}), 6.81(\mathrm{~d}$, $J=8.4 \mathrm{~Hz}, 2 \mathrm{H}$, aromatic $\mathrm{CH}), 5.60(\mathrm{t}, J=7.3 \mathrm{~Hz}, 1 \mathrm{H}, \mathrm{C}=\mathrm{CH}), 4.51(\mathrm{t}, J=6.6 \mathrm{~Hz}, 1$ $\mathrm{H}, \mathrm{CHOH}), 3.73\left(\mathrm{~s}, 3 \mathrm{H}, \mathrm{OCH}_{3}\right), 2.30$ (q, J=7.4 Hz, $\left.2 \mathrm{H}, \mathrm{C}=\mathrm{CHCH}_{2}\right), 1.96(\mathrm{~m}, 4 \mathrm{H}$, $\mathrm{CH}_{3}$ and $\mathrm{OH}$ ), $1.79,1.67,1.53$, and 1.40 (m each, $\left.1: 1: 1: 1 \mathrm{H}, \mathrm{CH}_{2} \mathrm{CH}_{2} \mathrm{CH}_{2} \mathrm{CHOH}\right) .{ }^{13} \mathrm{C}$ NMR $\left(100 \mathrm{MHz}, \mathrm{CDCl}_{3}\right) \delta 159.1,144.0,141.8,140.3,137.0$, and 136.1 (Cq), 132.0, $130.3,130.2,129.4,128.0,127.2,127.1,126.7,125.6$, and 113.9 (aromatic $\mathrm{CH}$ ), 74.0 $(\mathrm{CHOH}), 55.3\left(\mathrm{OCH}_{3}\right), 38.5,29.1$, and $26.4\left(\mathrm{CH}_{2} \mathrm{CH}_{2} \mathrm{CH}_{2}\right), 20.5\left(\mathrm{CH}_{3}\right)$. HRMS Calcd for $\mathrm{C}_{26} \mathrm{H}_{28} \mathrm{O}_{2},[\mathrm{M}+\mathrm{Na}]^{+}$: 395.1987; Found: 395.1965 .

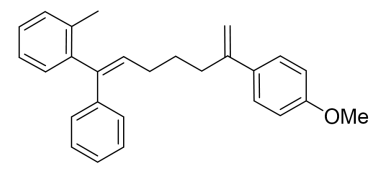

(E) -1-(6-(4-methoxyphenyl)-1-phenylhepta-1,6-dien-1-yl)-2-methylbenzene (10): 103 mg, 94\%; pale yellow liquid. ${ }^{1} \mathrm{H}$ NMR (400 MHz, $\left.\mathrm{CDCl}_{3}\right) \delta 7.25$ (m, $4 \mathrm{H}$, aromatic $\mathrm{CH}), 7.13(\mathrm{~m}, 6 \mathrm{H}$, aromatic $\mathrm{CH}), 7.06(\mathrm{~m}, 1 \mathrm{H}$, aromatic $\mathrm{CH}), 6.80(\mathrm{~m}, 2 \mathrm{H}$, aromatic $\mathrm{CH}), 5.62(\mathrm{t}, J=7.4 \mathrm{~Hz}, 1 \mathrm{H}, \mathrm{C}=\mathrm{CH}), 5.13\left(\mathrm{~d}, J=1.3 \mathrm{~Hz}, 1 \mathrm{H}, \mathrm{C}=\mathrm{CH}_{2}\right)$, $4.89\left(\mathrm{~d}, J=1.1 \mathrm{~Hz}, 1 \mathrm{H}, \mathrm{C}=\mathrm{CH}_{2}\right), 3.73\left(\mathrm{~s}, 3 \mathrm{H}, \mathrm{OCH}_{3}\right), 2.47(\mathrm{t}, J=7.6 \mathrm{~Hz}, 2 \mathrm{H}$, 
$\mathrm{CH}_{2} \mathrm{CH}_{2} \mathrm{CH}_{2}$ ), 2.30 (q, $\left.J=7.4 \mathrm{~Hz}, 2 \mathrm{H}, \mathrm{CH}_{2} \mathrm{CH}_{2} \mathrm{CH}_{2}\right), 1.98\left(\mathrm{~s}, 3 \mathrm{H}, \mathrm{CH}_{3}\right), 1.59$ (m, 2 $\left.\mathrm{H}, \mathrm{CH}_{2} \mathrm{CH}_{2} \mathrm{CH}_{2}\right) .{ }^{13} \mathrm{C} \mathrm{NMR}\left(100 \mathrm{MHz}, \mathrm{CDCl}_{3}\right) \delta 159.1,147.6,144.1,141.9,140.3$, 136.2, and 133.7 (Cq), 132.0, 130.3, 130.3, 129.4, 128.0, 127.3, 127.1, 126.7, 125.6, 113.7, and 111.0 (aromatic $\mathrm{CH}), 55.3\left(\mathrm{OCH}_{3}\right), 35.1,29.0$, and $28.9\left(\mathrm{CH}_{2} \mathrm{CH}_{2} \mathrm{CH}_{2}\right)$, 20.6 $\left(\mathrm{CH}_{3}\right)$. HRMS Calcd for $\mathrm{C}_{27} \mathrm{H}_{28} \mathrm{O},[\mathrm{M}+\mathrm{H}]^{+}: 369.2218$; Found: 369.2218. 


\section{Copies of NMR spectra}

WQN-1630W

$1 \mathrm{H}$ NMR in $\mathrm{CDCl3}(400 \mathrm{MHz})$

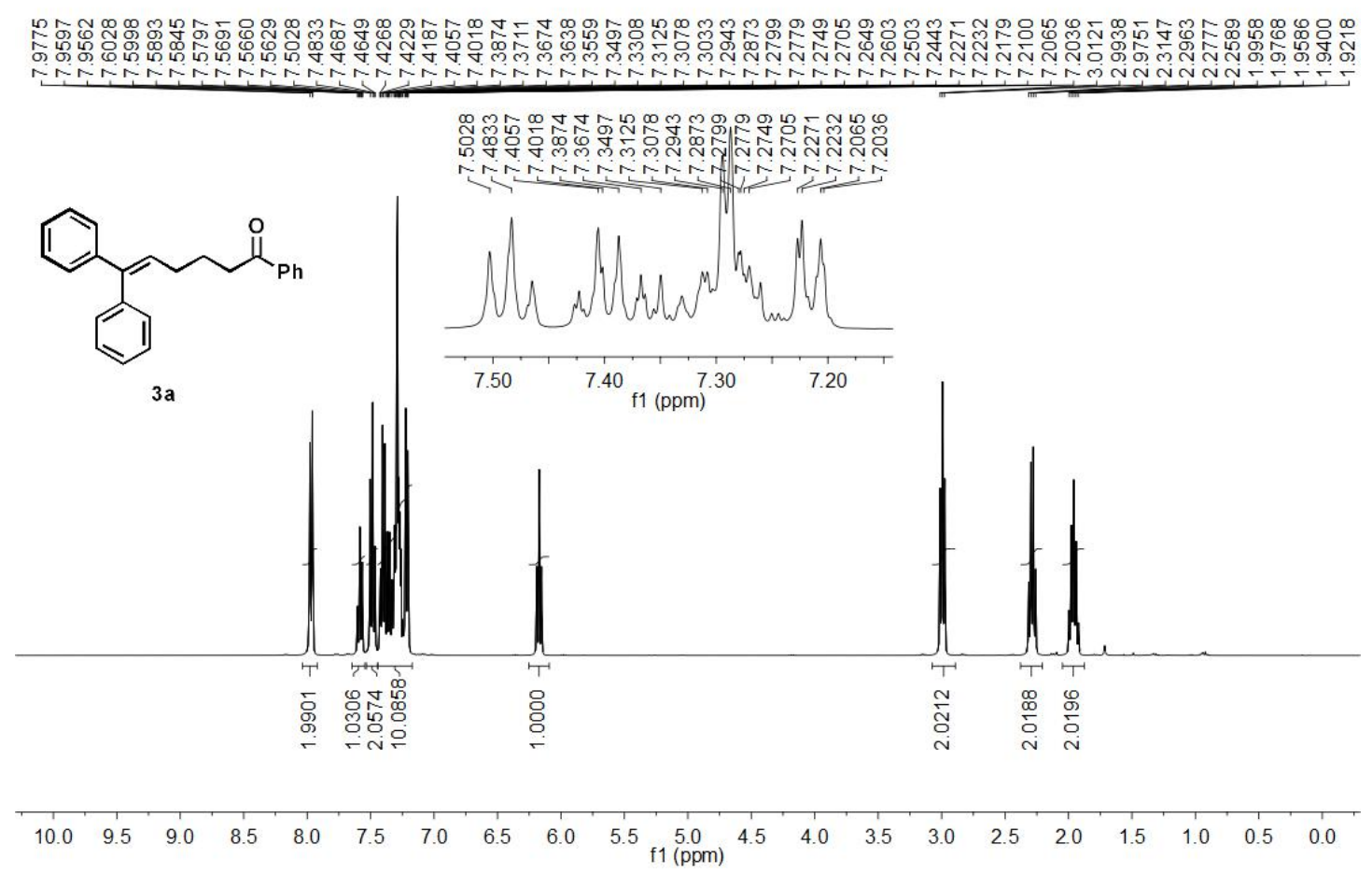

WQN-1630W

13C NMR in $\mathrm{CDCl} 3(100 \mathrm{MHz})$

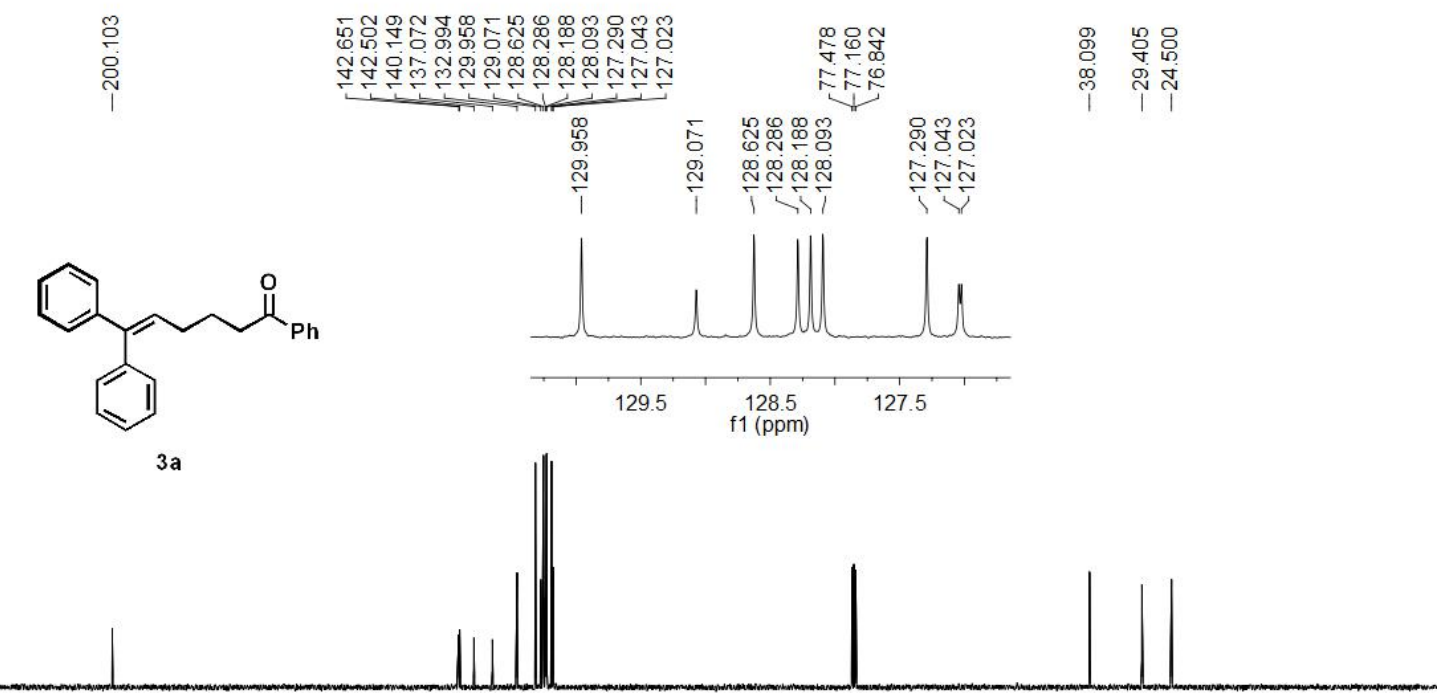

$\begin{array}{lllllllllllllllllllllll}210 & 200 & 190 & 180 & 170 & 160 & 150 & 140 & 130 & 120 & 110_{\mathrm{f} 1(\mathrm{ppm})} & 90 & 80 & 70 & 60 & 50 & 40 & 30 & 20 & 10 & 0 & -10\end{array}$ 
WQN-1642W

PROTON CDC13 \{D: \NMR400\203\} nmr 49

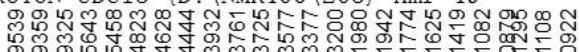

두응

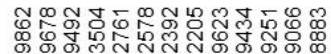

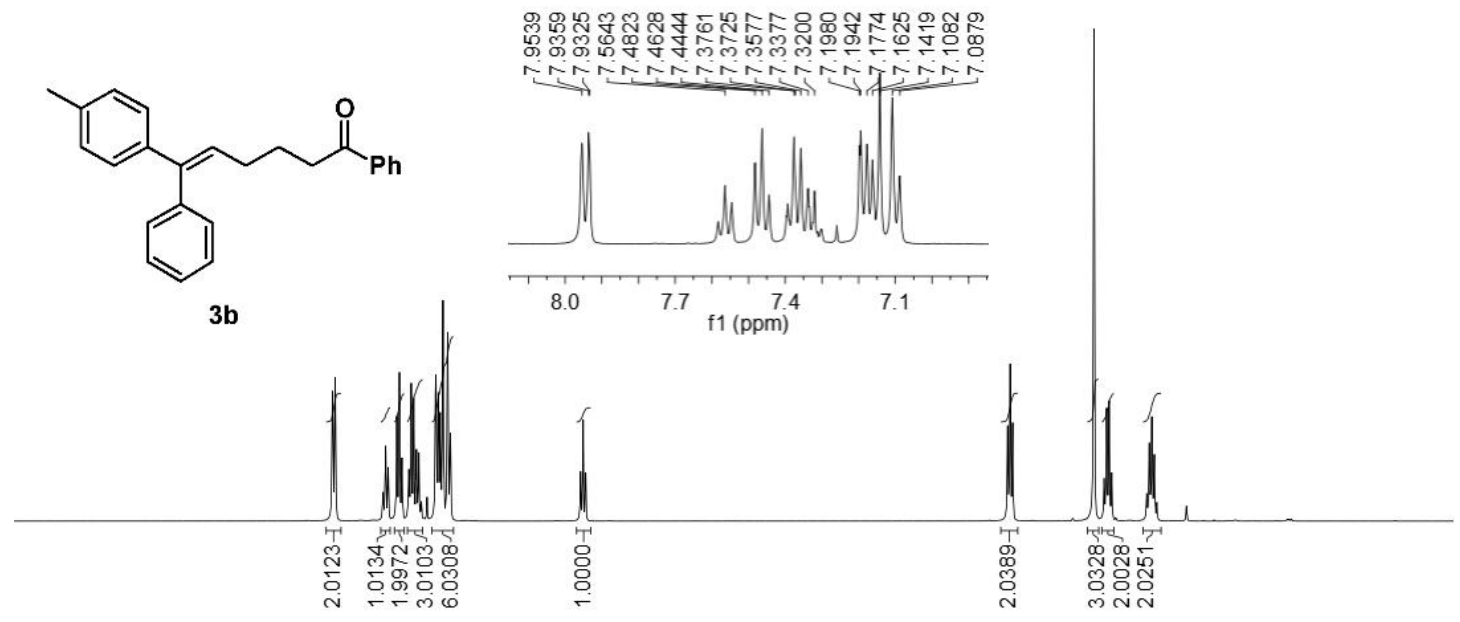

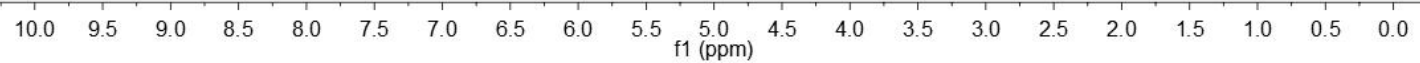

WQN-1642W

C13CPD CDC13 \{D: \NMR400\203\} nmr 49

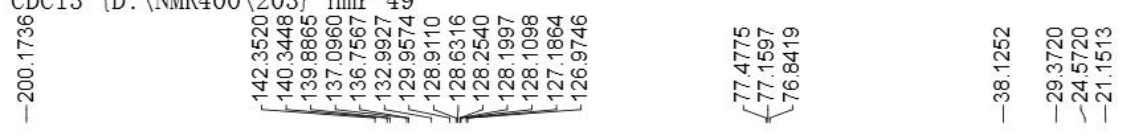

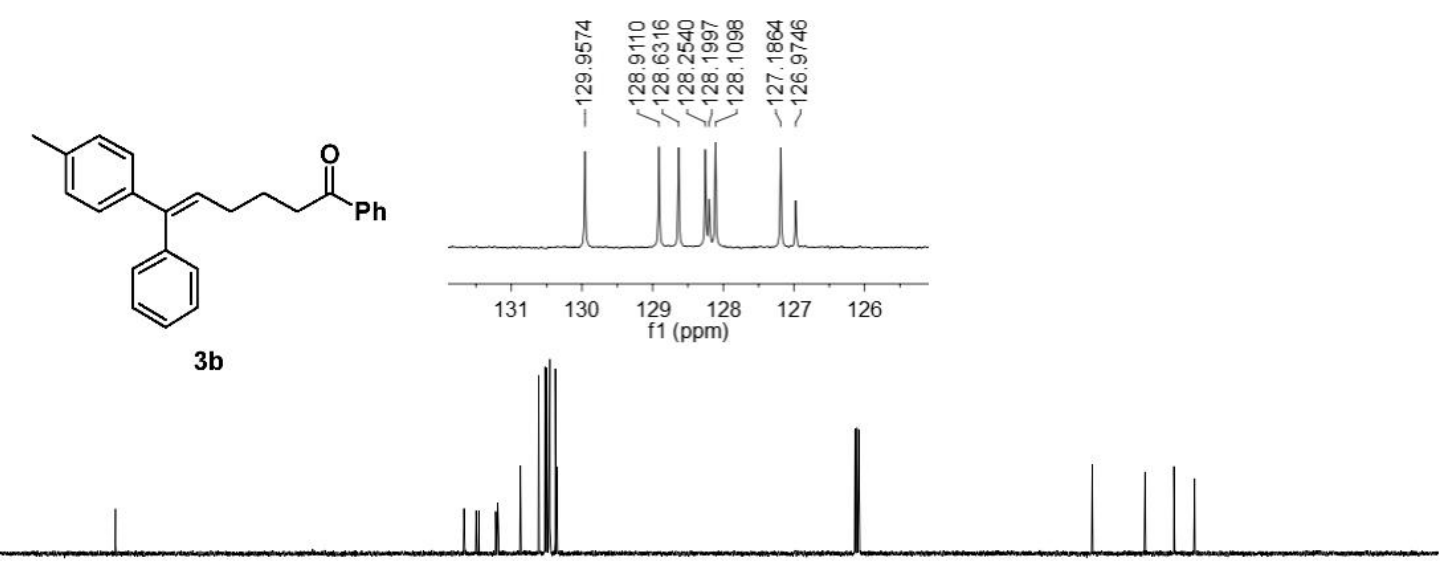

$\begin{array}{llllllllllllllllllllll}210 & 200 & 190 & 180 & 170 & 160 & 150 & 140 & 130 & 120 & 110 \begin{array}{c}100 \\ \mathrm{f} 1(\mathrm{ppm})\end{array} & 90 & 80 & 70 & 60 & 50 & 40 & 30 & 20 & 10 & 0 & -10\end{array}$ 
WQN-1655W

PROTON CDC13 \{D: \NMR400\203\} nmr 34

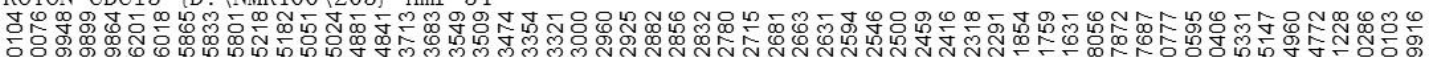

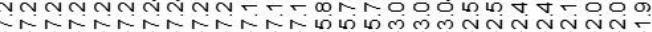

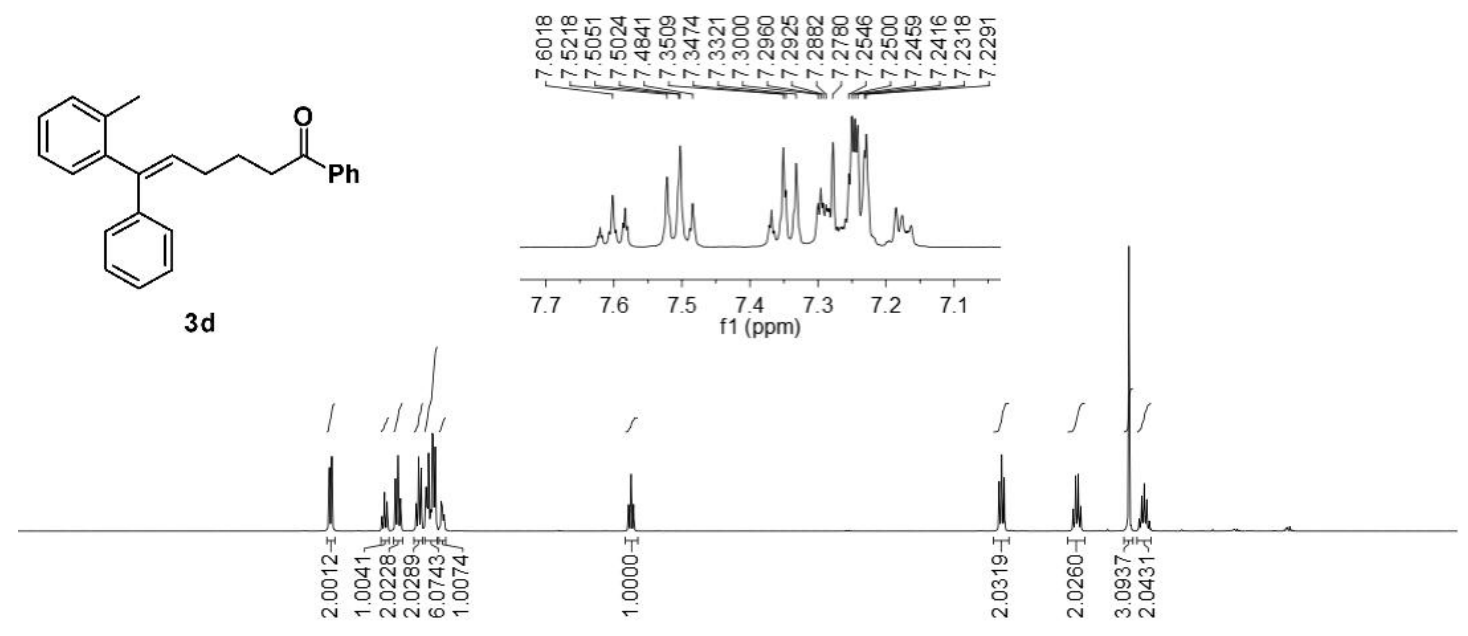

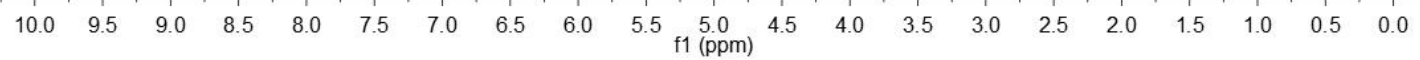

WQN-1655W

C13CPD CDC13 \{D: \NMR400\203\} nmr 34

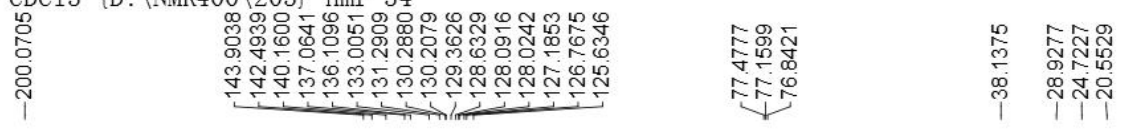

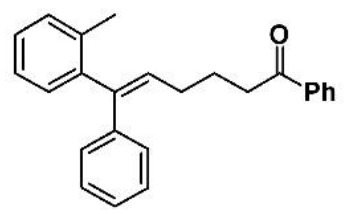



i

$3 d$

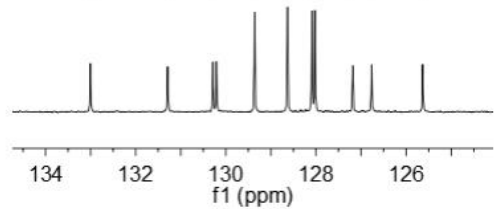

$\begin{array}{lllllllllllllllllllllll}210 & 200 & 190 & 180 & 170 & 160 & 150 & 140 & 130 & 120 & 110 & \begin{array}{c}100 \\ \mathrm{f} 1(\mathrm{ppm})\end{array} & 90 & 80 & 70 & 60 & 50 & 40 & 30 & 20 & 10 & 0 & -10\end{array}$ 
WQN-1646W
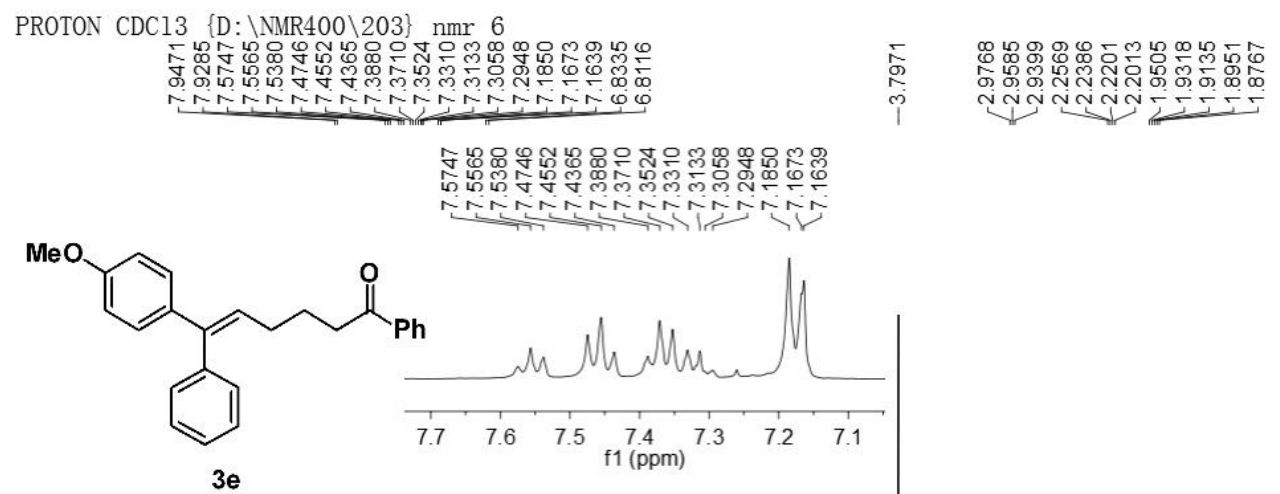

$3 e$



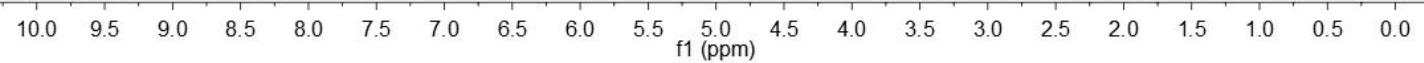

WQN-1646W

C13CPD CDC13 \{D: \NMR400\203\} nmr 6

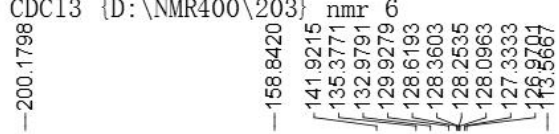

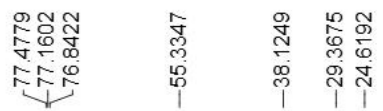<smiles>COc1ccc(/C(=C/CCCC(=O)c2ccccc2)c2ccccc2)cc1</smiles>

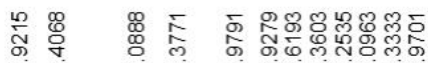

莎
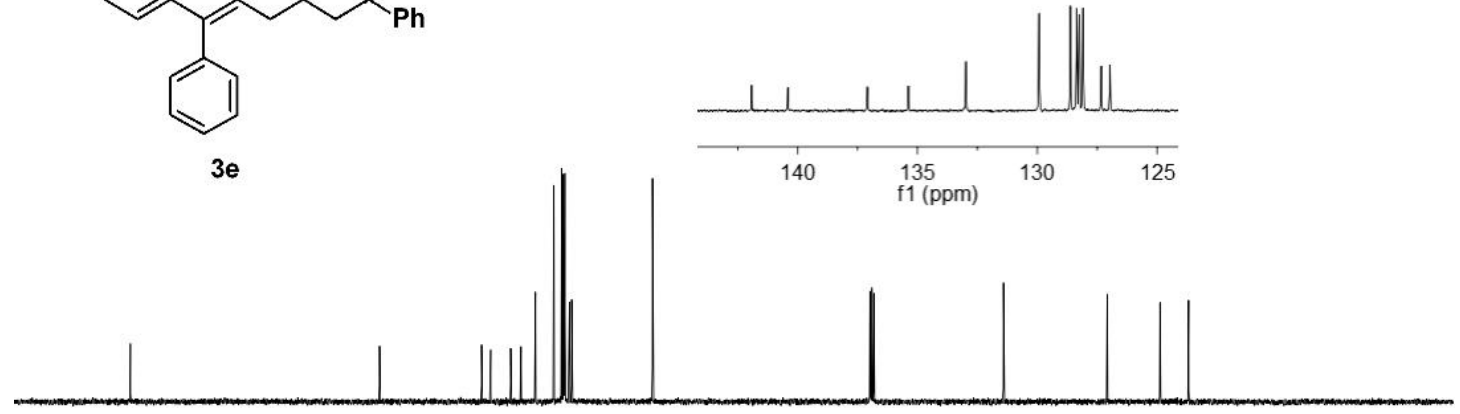

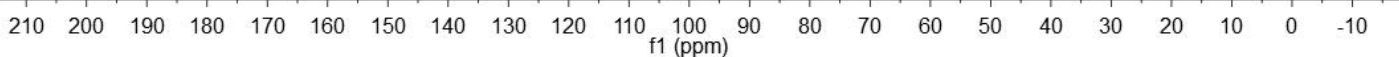


WQN-1669W

1H NMR in $\mathrm{CDCl} 3(400 \mathrm{MHz})$

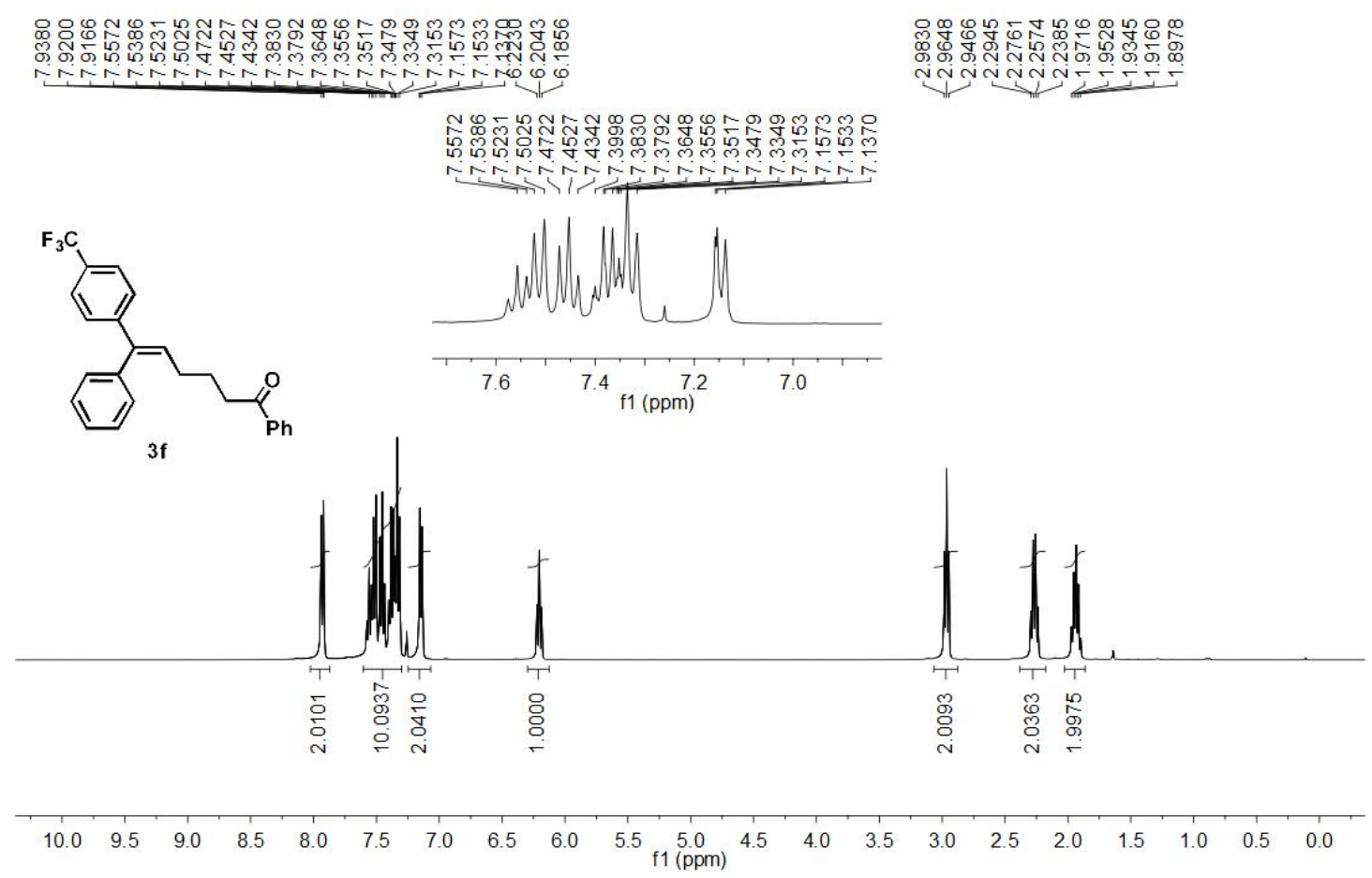

WQN-1669W

13C NMR in $\mathrm{CDCl} 3(100 \mathrm{MHz})$

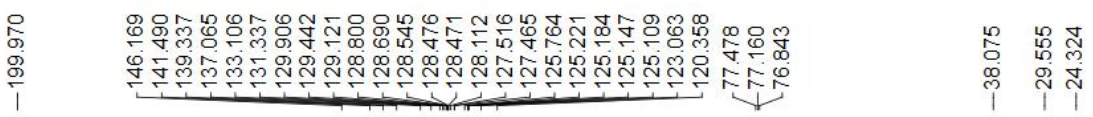

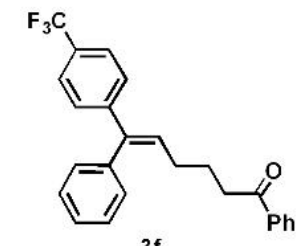

$\begin{array}{lllllllllllllllllllllll}210 & 200 & 190 & 180 & 170 & 160 & 150 & 140 & 130 & 120 & 110 & 100 & 90 & 80 & 70 & 60 & 50 & 40 & 30 & 20 & 10 & 0 & -10\end{array}$ 
WQN-1662W

1H NMR in $\mathrm{CDCl} 3(400 \mathrm{MHz})$

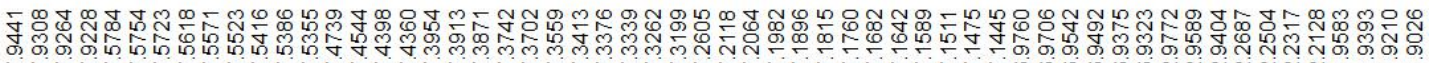 \\ ninting}

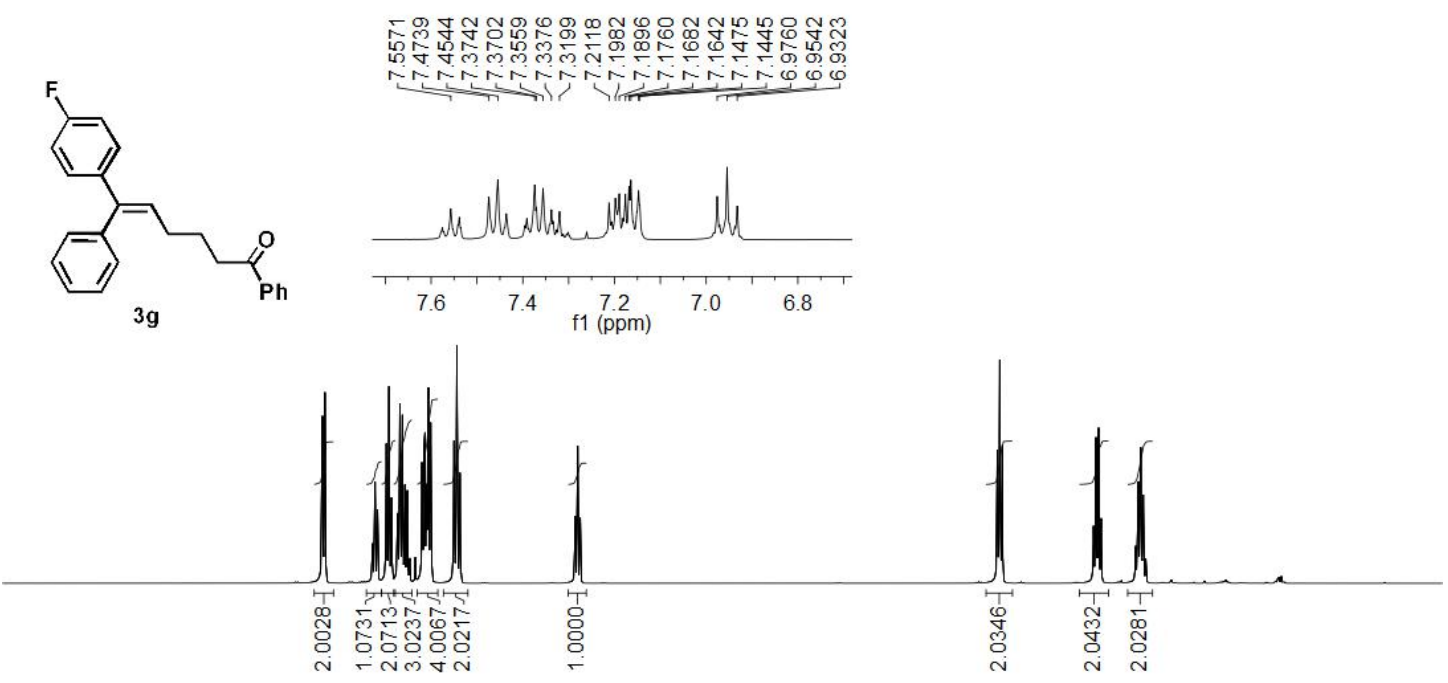

$\begin{array}{lllllllllllllllllllll}10.0 & 9.5 & 9.0 & 8.5 & 8.0 & 7.5 & 7.0 & 6.5 & 6.0 & 5.5 & \begin{array}{c}5.0 \\ (\mathrm{ppm})\end{array} & 4.5 & 4.0 & 3.5 & 3.0 & 2.5 & 2.0 & 1.5 & 1.0 & 0.5 & 0.0\end{array}$

WQN-1662W

13C NMR in $\mathrm{CDCl} 3(100 \mathrm{MHz})$

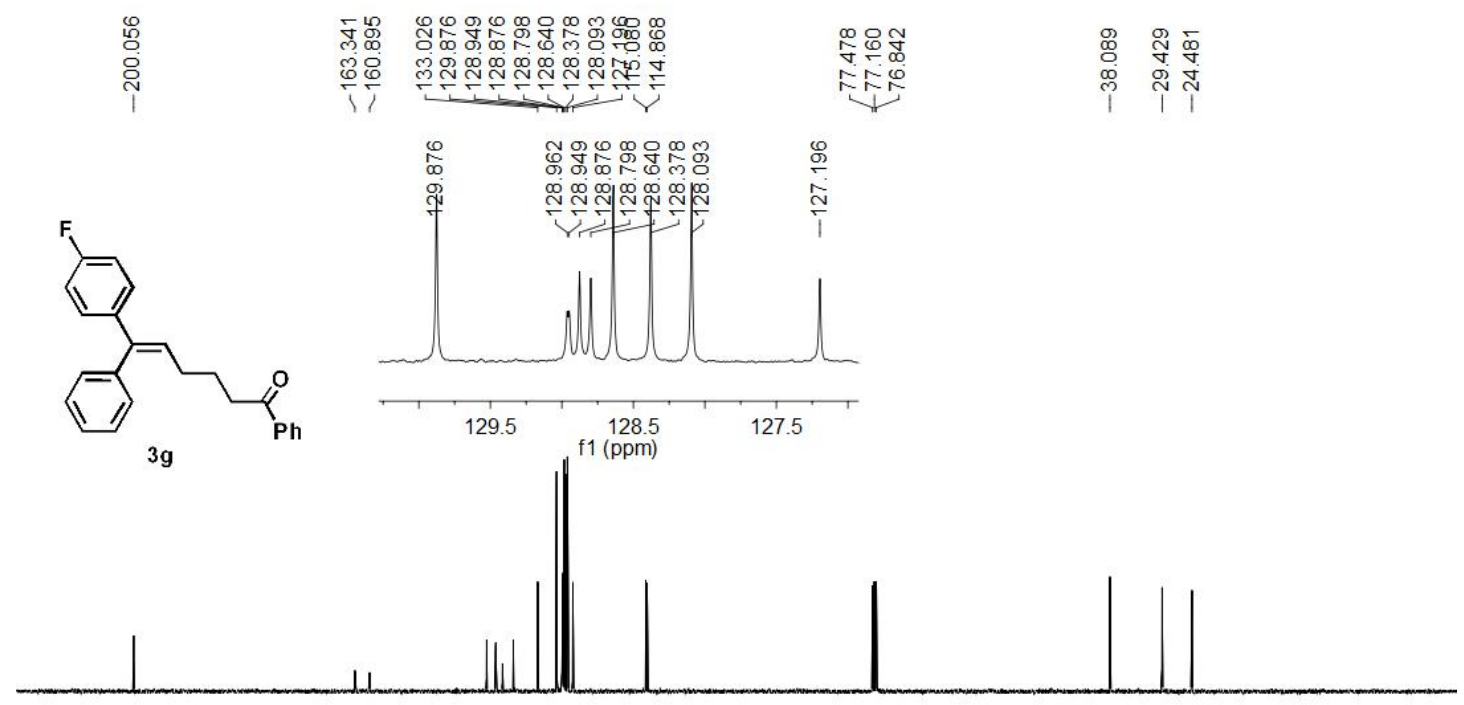

$\begin{array}{lllllllllllllllllllllllll}210 & 200 & 190 & 180 & 170 & 160 & 150 & 140 & 130 & 120 & 110 & 100 & 90 & 80 & 70 & 60 & 50 & 40 & 30 & 20 & 10 & 0 & -10\end{array}$ 
WQN-1665W

1H NMR in $\mathrm{CDCl} 3(400 \mathrm{MHz})$

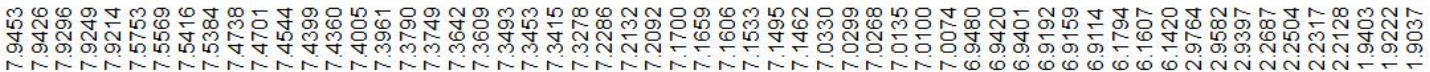

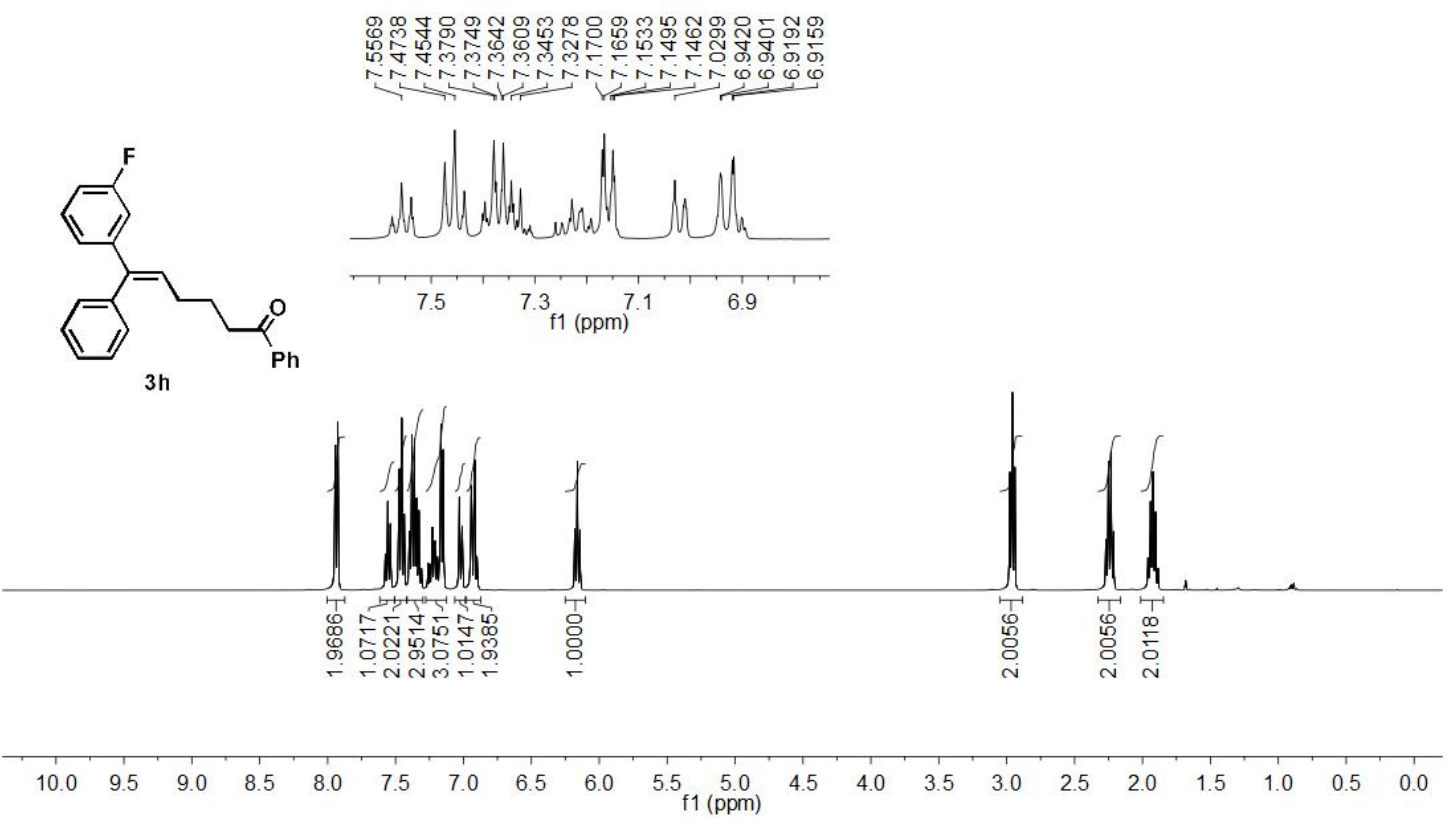

WQN-1665W

$13 \mathrm{C}$ NMR in $\mathrm{CDCl} 3(100 \mathrm{MHz})$

\begin{tabular}{|c|c|c|c|c|}
\hline 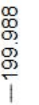 & $\begin{array}{l}\text { 웅 } \\
\frac{6}{6} \\
\frac{6}{6} \\
\frac{6}{1}\end{array}$ & 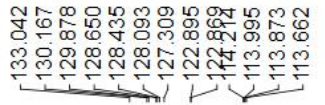 & 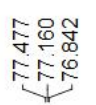 & 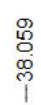 \\
\hline
\end{tabular}

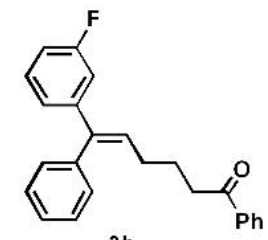

$\mathrm{Ph}$

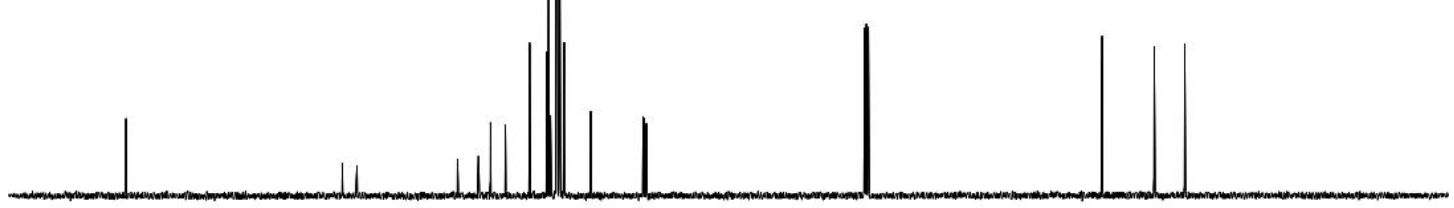

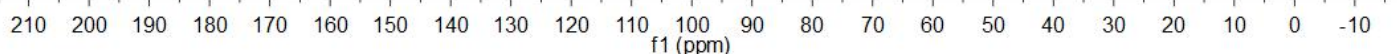


WQN-1647W

1H NMR in $\mathrm{CDCl} 3(400 \mathrm{MHz})$

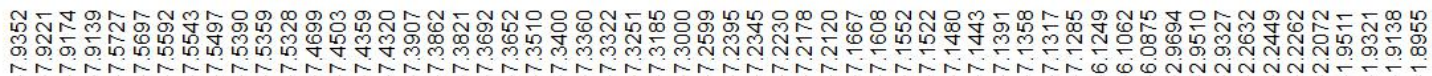
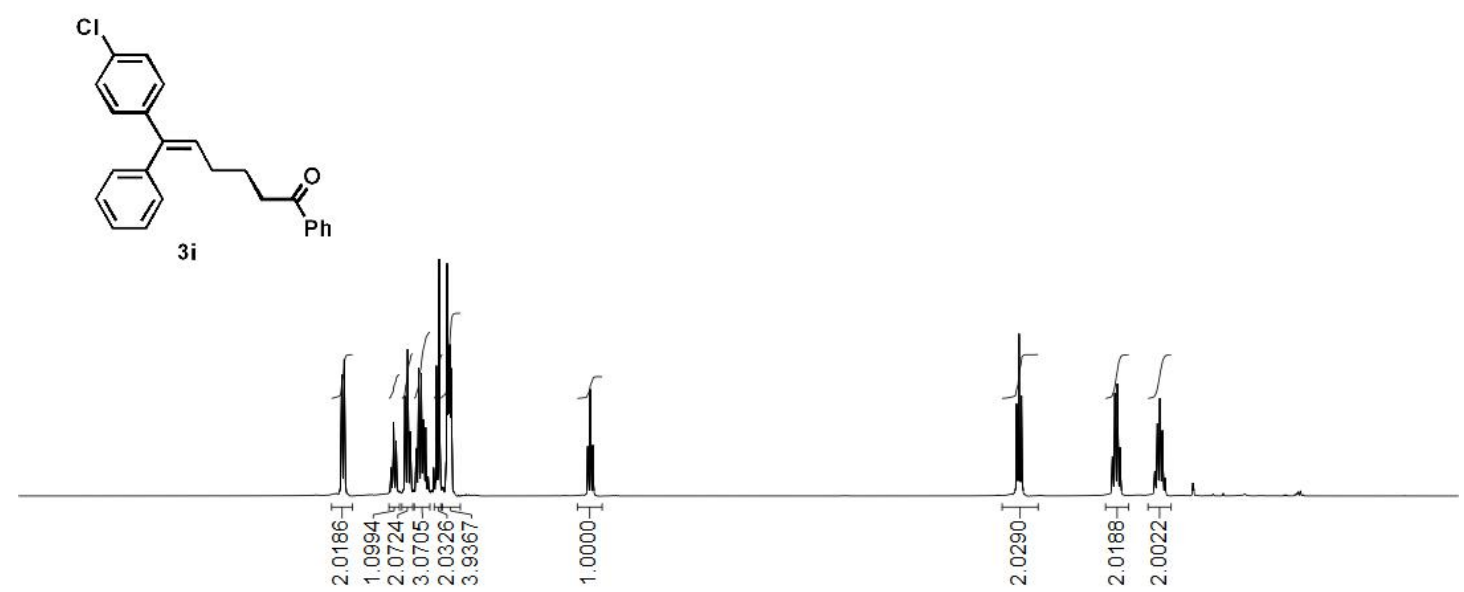

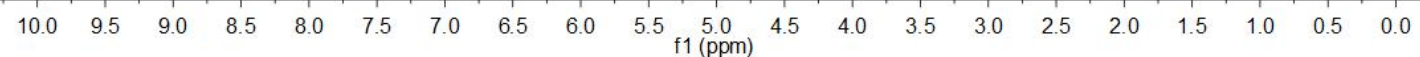

WQN-1647W

$13 \mathrm{C}$ NMR in $\mathrm{CDCl} 3(100 \mathrm{MHz})$

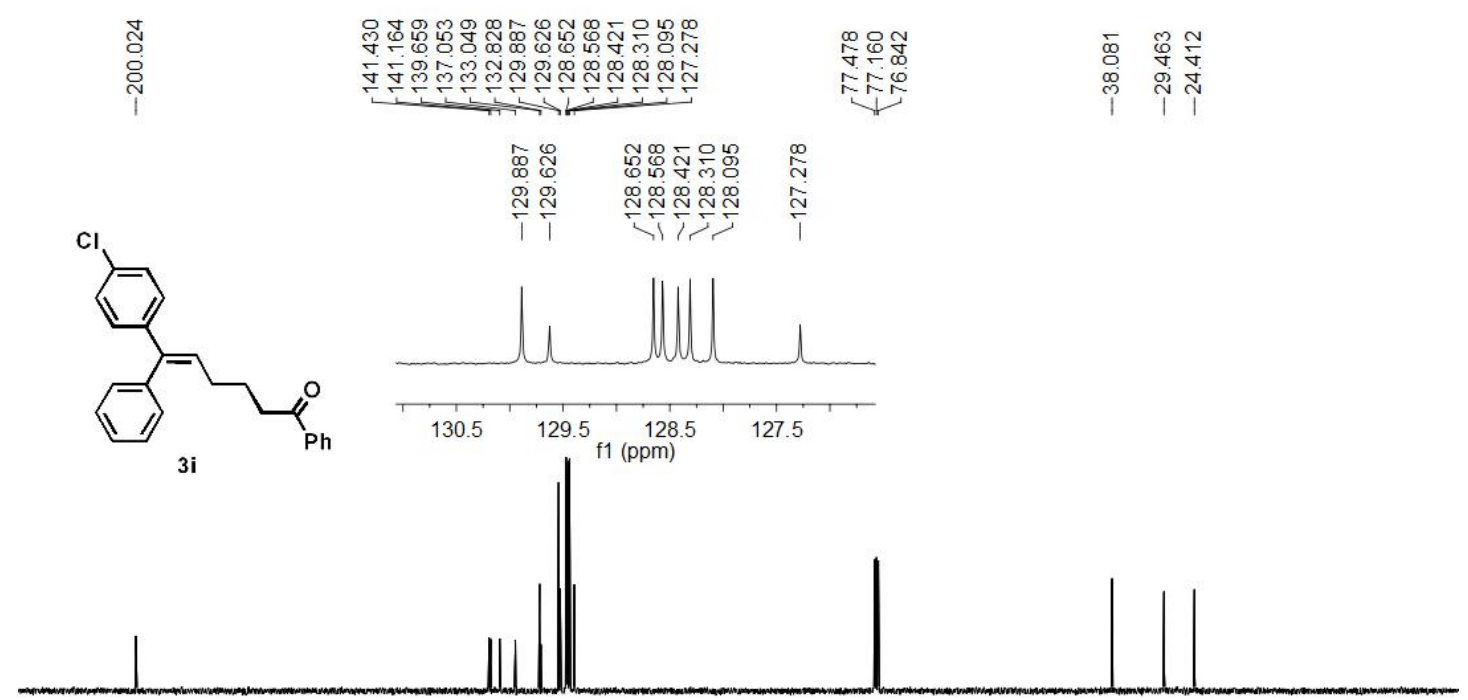

$\begin{array}{llllllllllllllllllllll}210 & 200 & 190 & 180 & 170 & 160 & 150 & 140 & 130 & 120 & 110 \begin{array}{l}100 \\ \mathrm{f} 1(\mathrm{ppm})\end{array} & 90 & 80 & 70 & 60 & 50 & 40 & 30 & 20 & 10 & 0 & -10\end{array}$ 
WQN-1642W

1H NMR in $\mathrm{CDCl} 3(400 \mathrm{MHz})$
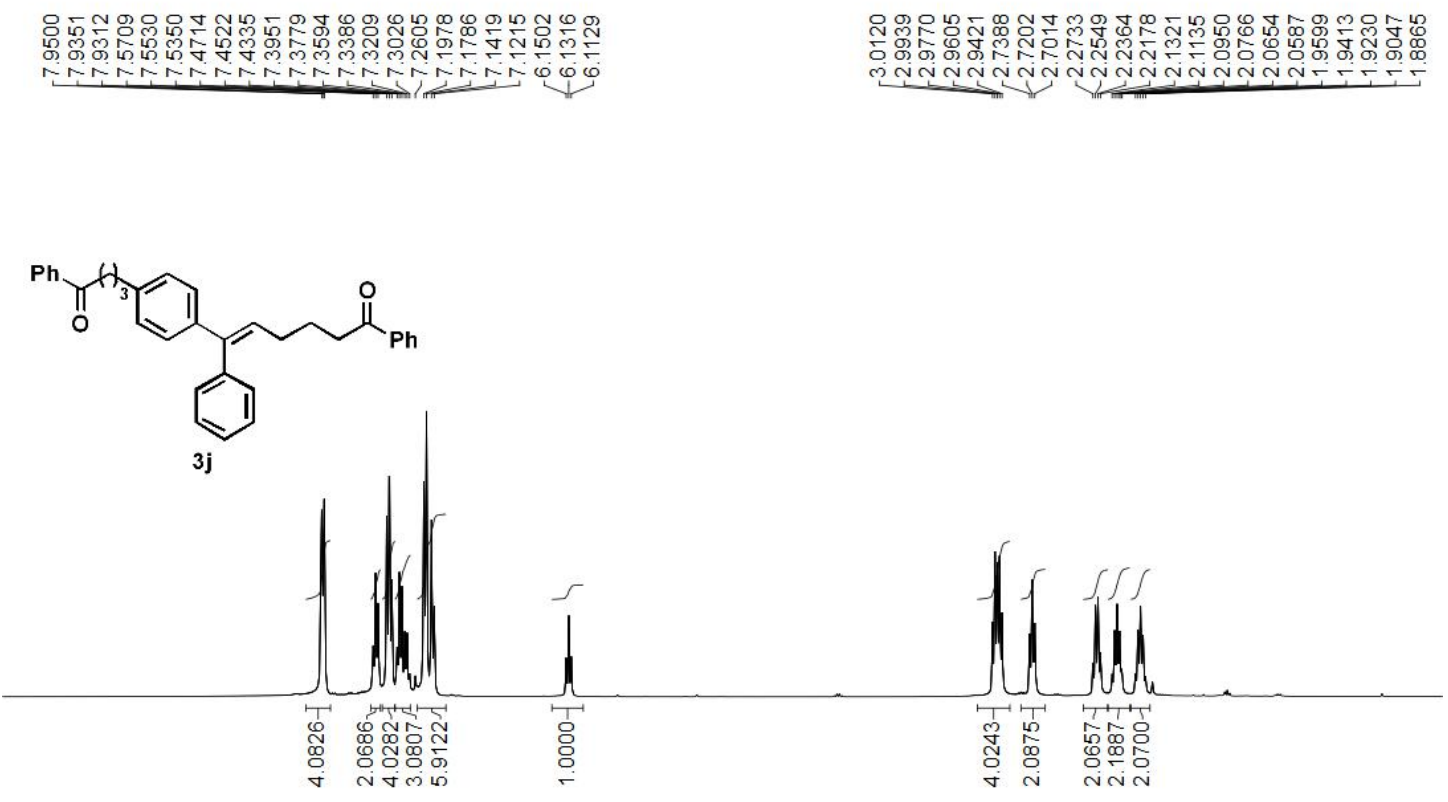

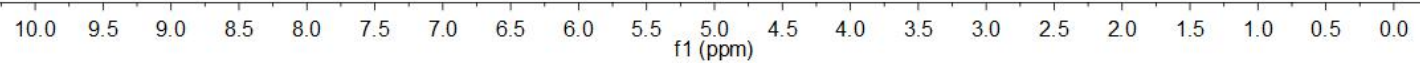

WQN-1624W-2

$13 \mathrm{C}$ NMR in $\mathrm{CDCl} 3(100 \mathrm{MHz})$
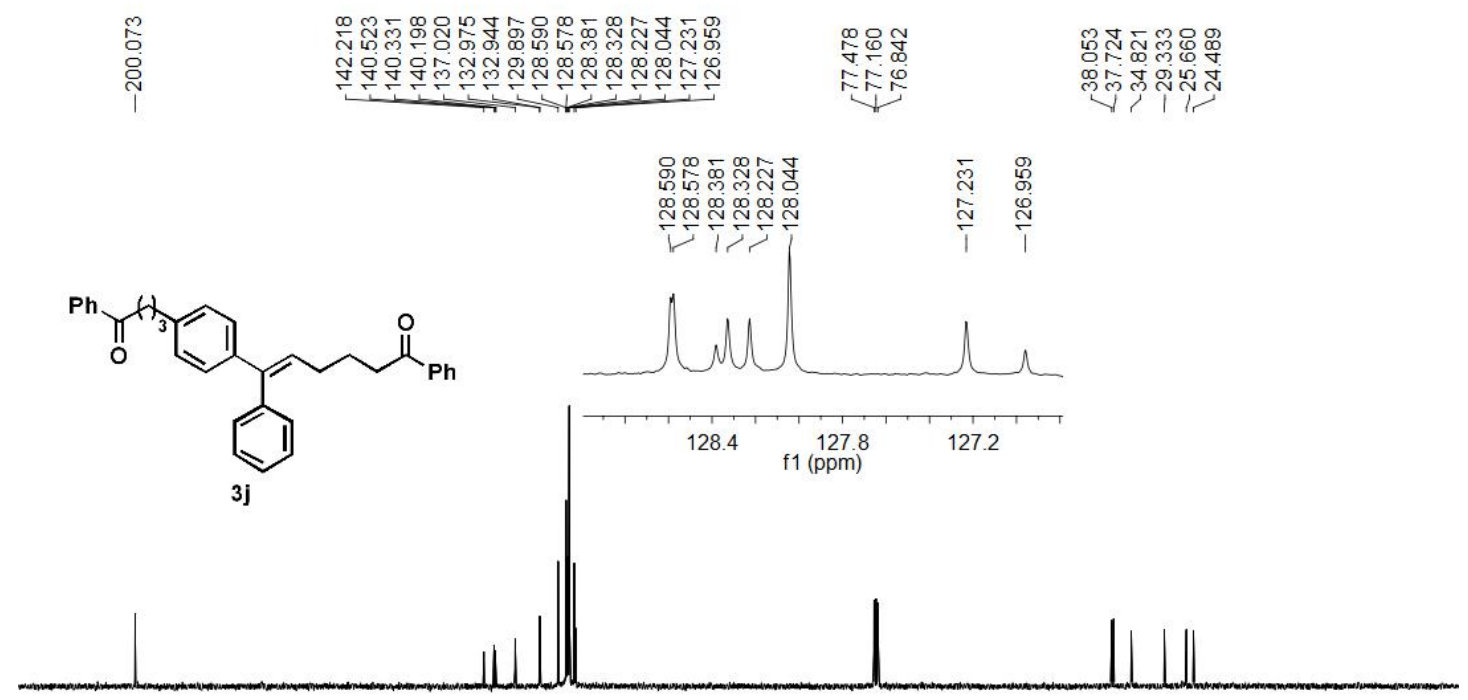

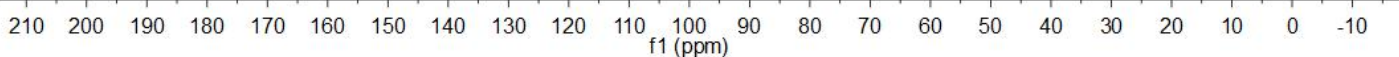


WQN-1670W

1H NMR in $\mathrm{CDCl} 3(400 \mathrm{MHz})$

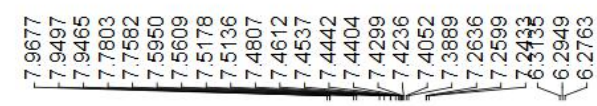

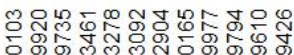

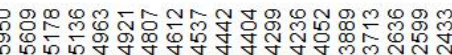

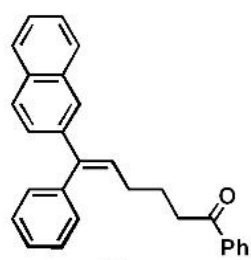

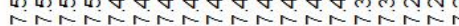

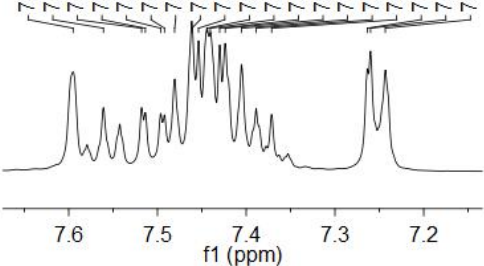

$1(\mathrm{ppm})$

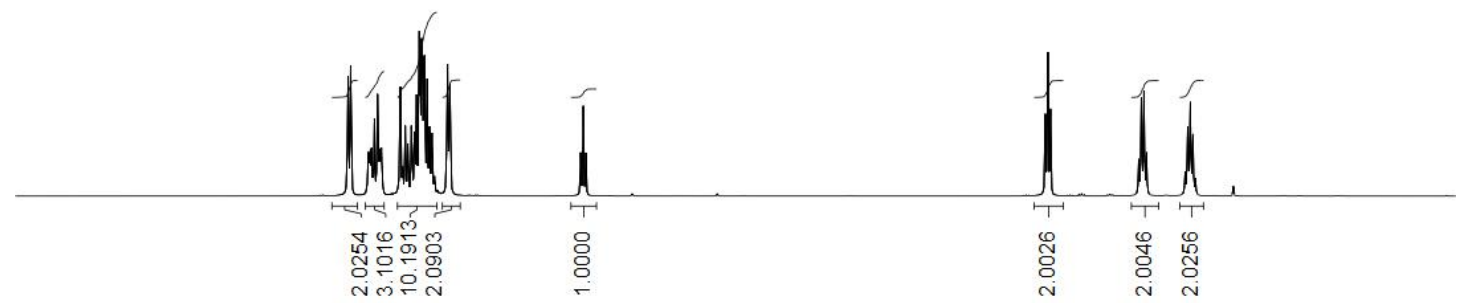

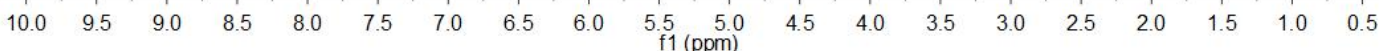

WQN-1670W

$13 \mathrm{C}$ NMR in $\mathrm{CDCl} 3(100 \mathrm{MHz})$

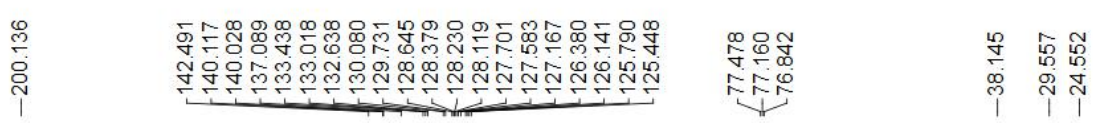

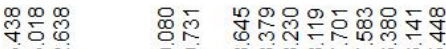

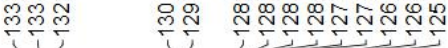
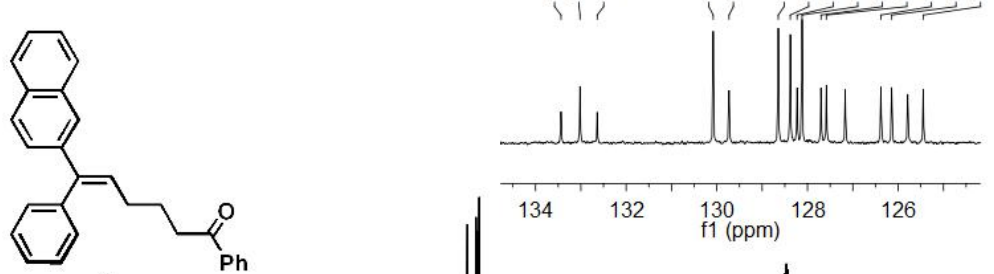

$\begin{array}{lllllllllllllllllllllll}210 & 200 & 190 & 180 & 170 & 160 & 150 & 140 & 130 & 120 & 110 & 100 & 90 & 80 & 70 & 60 & 50 & 40 & 30 & 20 & 10 & 0 & -10\end{array}$ 
WQN-1661W

1H NMR in $\mathrm{CDCl} 3(400 \mathrm{MHz})$
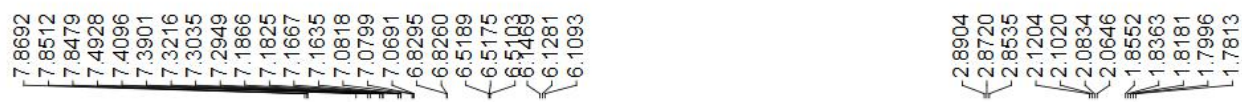

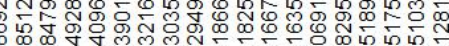

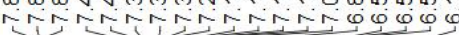
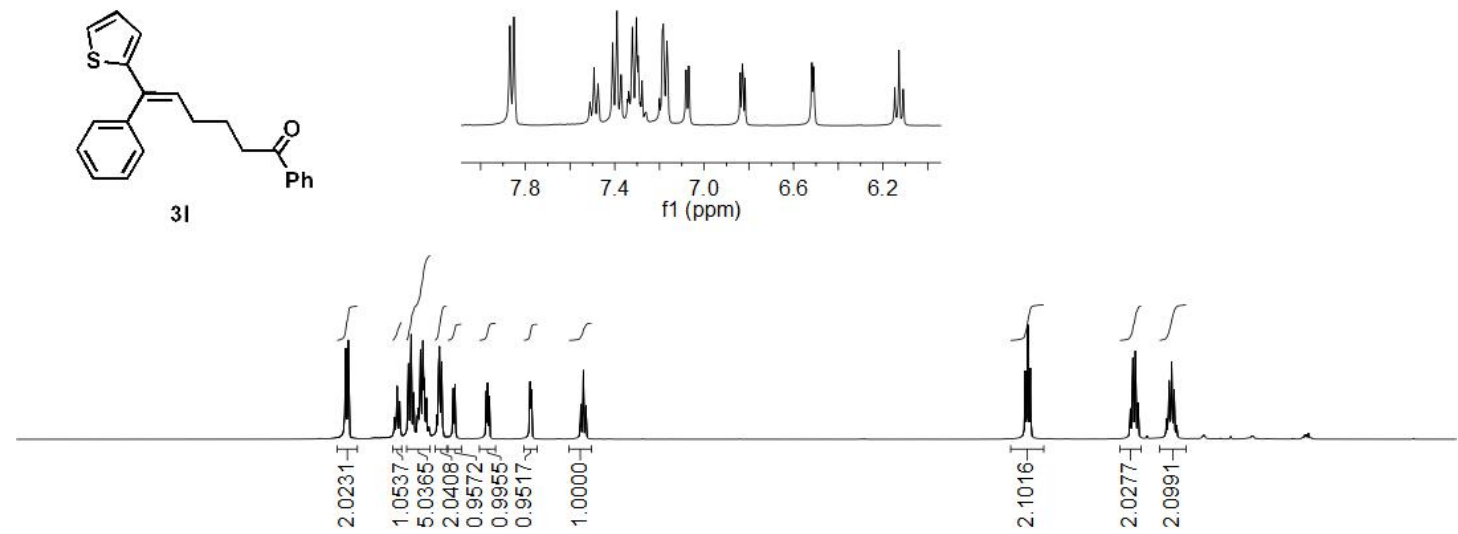

$\begin{array}{llllllllllllllllllll}10.0 & 9.5 & 9.0 & 8.5 & 8.0 & 7.5 & 7.0 & 6.5 & 6.0 & 5.5 \underset{\mathrm{f} 1}{\mathrm{f}(\mathrm{ppm})} \mathbf{4 . 5} & 4.0 & 3.5 & 3.0 & 2.5 & 2.0 & 1.5 & 1.0 & 0.5 & 0.0\end{array}$

WQN-1661W

$13 \mathrm{C}$ NMR in $\mathrm{CDCl} 3(100 \mathrm{MHz})$

章

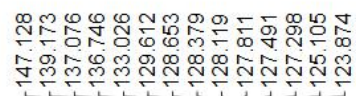

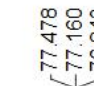

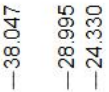

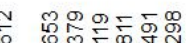

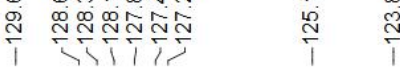

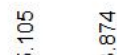
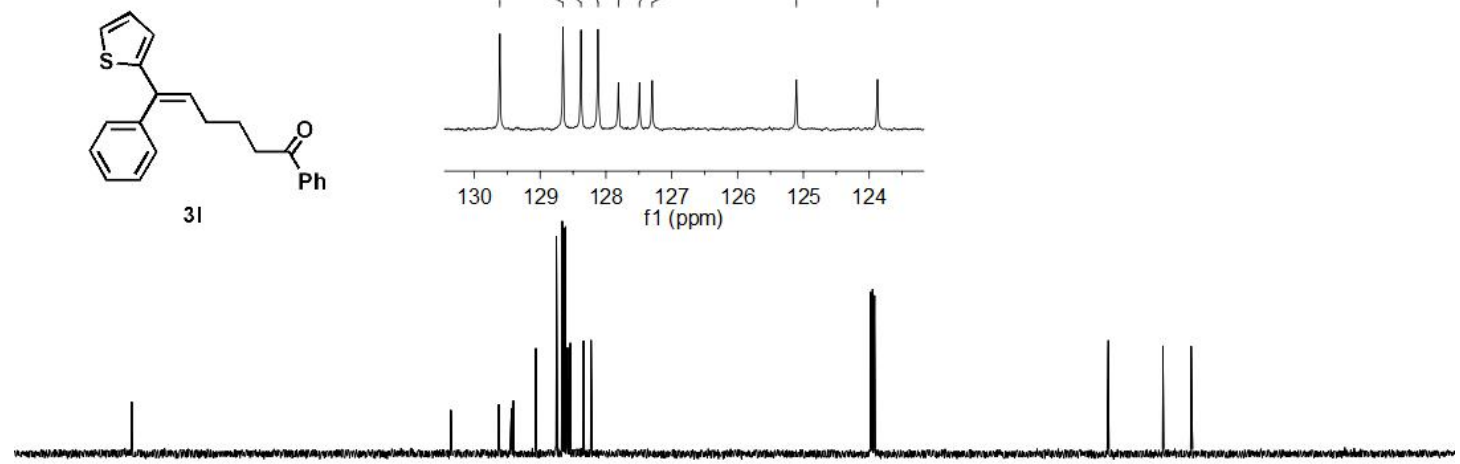

$\begin{array}{llllllllllllllllllllll}210 & 200 & 190 & 180 & 170 & 160 & 150 & 140 & 130 & 120 & 110 \begin{array}{l}100 \\ \mathrm{f} 1(\mathrm{ppm})\end{array} & 90 & 80 & 70 & 60 & 50 & 40 & 30 & 20 & 10 & 0 & -10\end{array}$ 
WQN-1687W

PROTON CDC13 \{D: \NMR400\203\} nmr 52

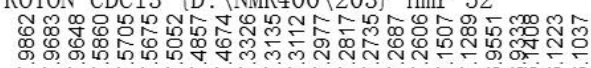



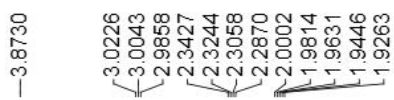

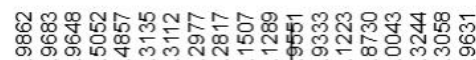<smiles>COc1ccc(C(=CCCCC(=O)c2ccccc2)c2ccccc2)cc1</smiles>

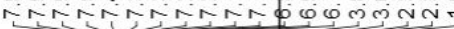

$3 m$
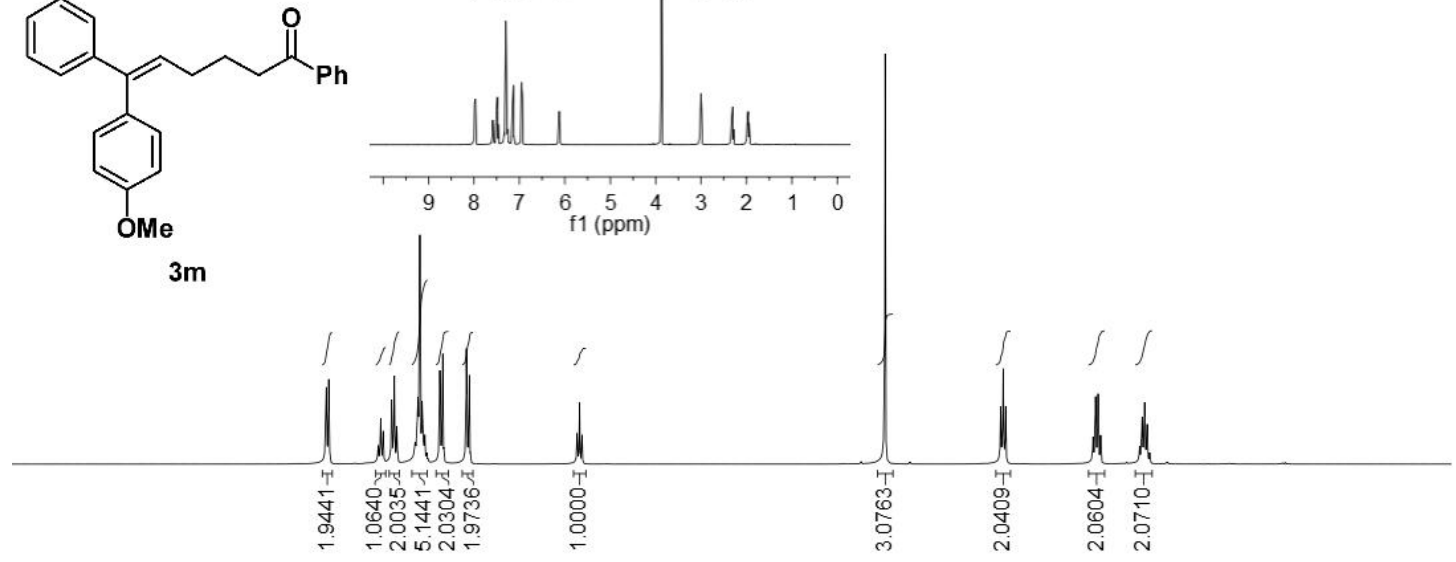

$\begin{array}{lllllllllllllllllllll}10.0 & 9.5 & 9.0 & 8.5 & 8.0 & 7.5 & 7.0 & 6.5 & 6.0 & 5.5 & \begin{array}{l}5.0 \\ \mathrm{f} 1(\mathrm{ppm})\end{array} & 4.5 & 4.0 & 3.5 & 3.0 & 2.5 & 2.0 & 1.5 & 1.0 & 0.5 & 0.0\end{array}$

WQN-1687W

C13CPD CDC13 \{D: \NMR400\203\} nmr 52

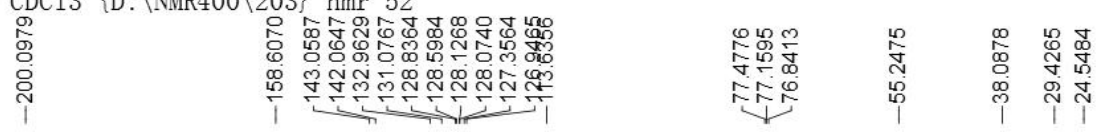

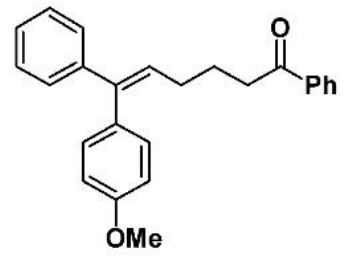

$3 m$

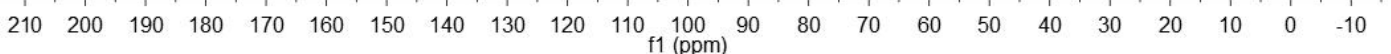


WQN-1689w

PROTON CDC13 \{D: \NMR400\203\} nmr 60

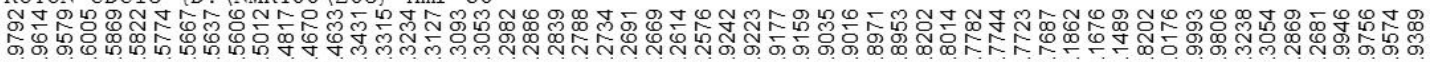



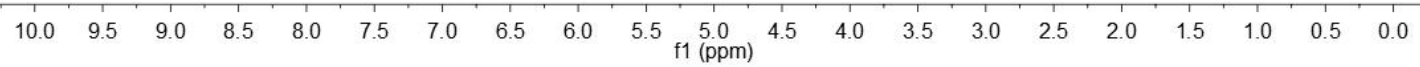

WQN-1689w

C13CPD CDC13 \{D: \NMR400\203\} nmr 60

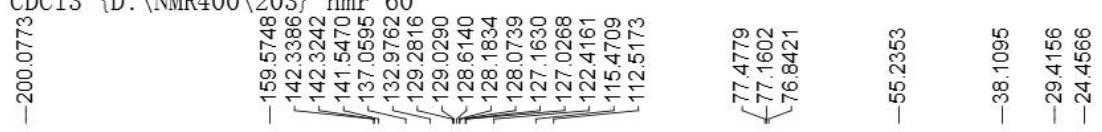



$3 n$

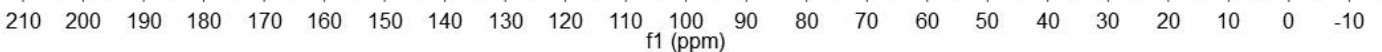


WQN-1686W

PROTON CDC13 \{D: \NMR400\203\} nmr 53

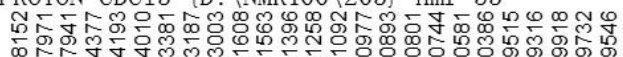

or v v

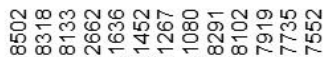

nin<smiles>CCCC(=O)CCCC=C(c1ccccc1)c1ccc(C)cc1</smiles>

30

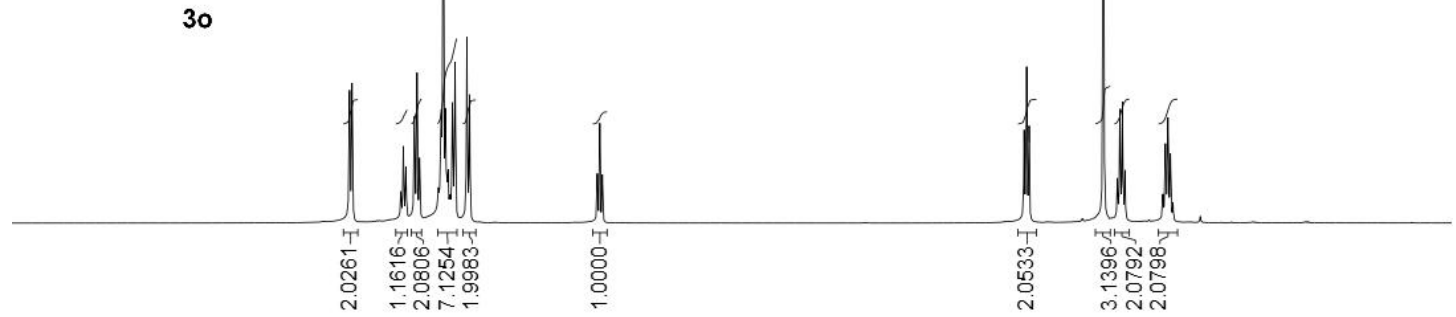

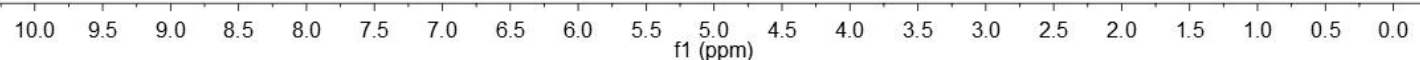

WQN-1686W

C13CPD CDC13 \{D: \NMR400\203\} nmr 53

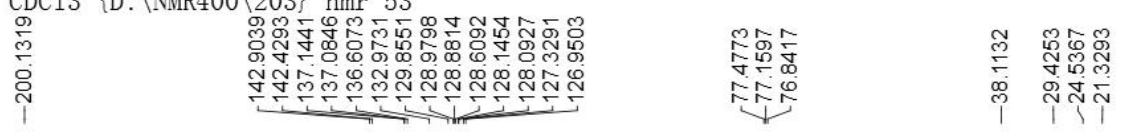<smiles>Cc1ccc(C(=CCCCC(=O)c2ccccc2)c2ccccc2)cc1</smiles>

30

$\begin{array}{lllllllllllllllllllllll}210 & 200 & 190 & 180 & 170 & 160 & 150 & 140 & 130 & 120 & 110 \begin{array}{c}100 \\ \mathrm{f} 1(\mathrm{ppm})\end{array} & 90 & 80 & 70 & 60 & 50 & 40 & 30 & 20 & 10 & 0 & -10\end{array}$ 
wqn-1680w

PROTON CDC13 \{D: \NMR400\203\} nmr 43

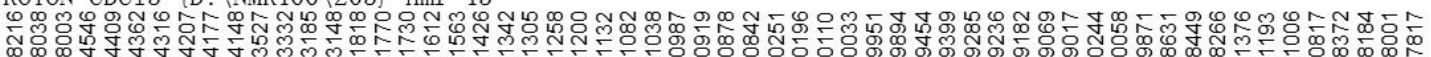

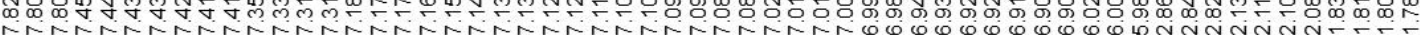<smiles>O=C(CCCC=C(c1ccccc1)c1ccccc1)c1ccccc1</smiles>

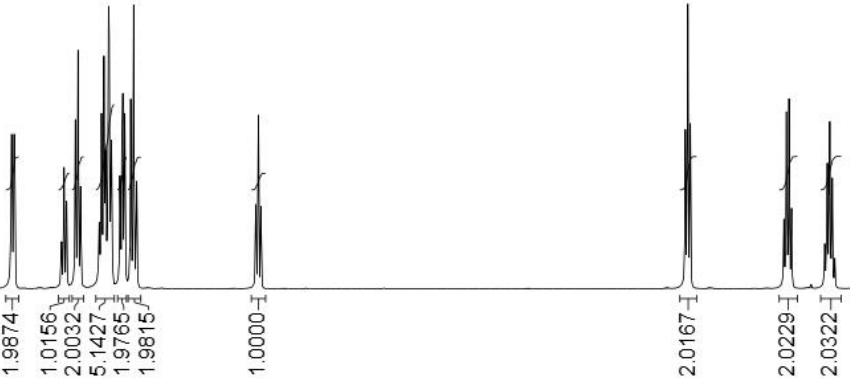

$\begin{array}{lllllllllllllllllllll}10.0 & 9.5 & 9.0 & 8.5 & 8.0 & 7.5 & 7.0 & 6.5 & 6.0 & 5.5 & \begin{array}{c}5.0 \\ \mathrm{f} 1\end{array}(\mathrm{ppm}) & 4.5 & 4.0 & 3.5 & 3.0 & 2.5 & 2.0 & 1.5 & 1.0 & 0.5 & 0.0\end{array}$

wqn-1680w

C13CPD CDC13 \{D: \NMR400\203\} nmr 43

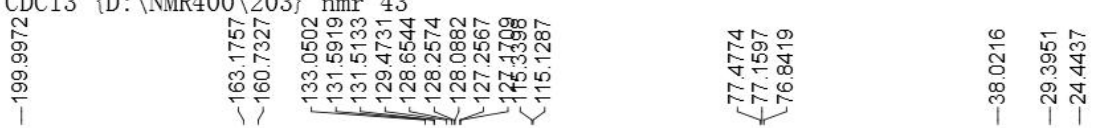<smiles>O=C(CCCC=C(c1ccccc1)c1ccccc1)c1ccc(F)cc1</smiles>

$3 p$

$\begin{array}{lllllllllllllllllllllll}210 & 200 & 190 & 180 & 170 & 160 & 150 & 140 & 130 & 120 & 110 & \begin{array}{c}100 \\ \mathrm{f} 1(\mathrm{ppm})\end{array} & 90 & 80 & 70 & 60 & 50 & 40 & 30 & 20 & 10 & 0 & -10\end{array}$ 
WQN-1718W

$1 \mathrm{H}$ NMR in $\mathrm{CDCl} 3(400 \mathrm{MHz})$

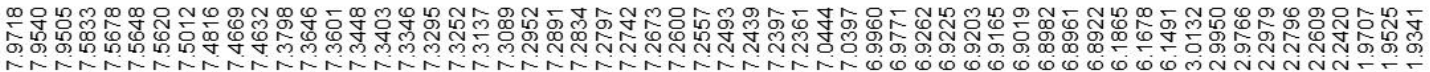

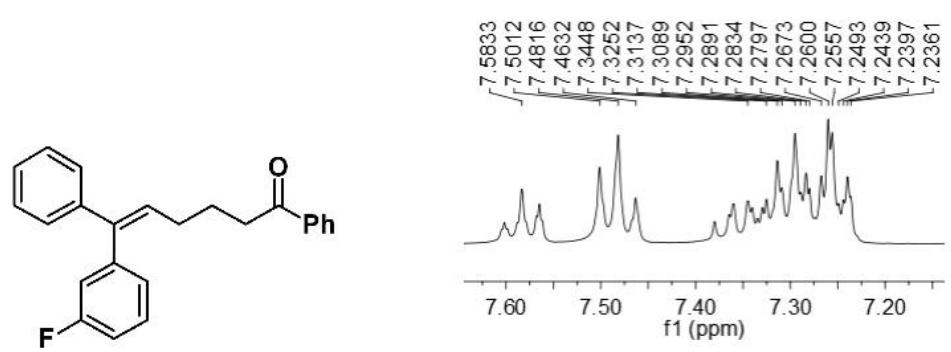

$3 q$

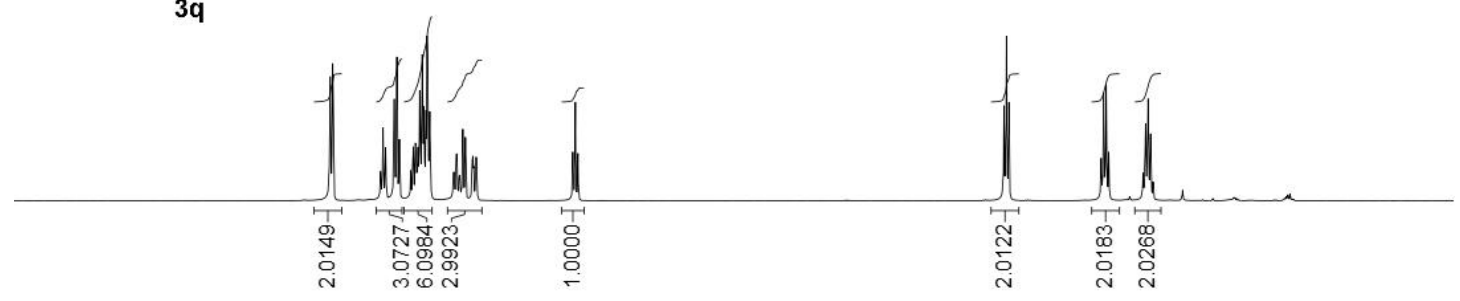

$\begin{array}{lllllllllllllllllllll}10.0 & 9.5 & 9.0 & 8.5 & 8.0 & 7.5 & 7.0 & 6.5 & 6.0 & 5.5 & \begin{array}{c}5.0 \\ \mathrm{fpm})\end{array} & 4.5 & 4.0 & 3.5 & 3.0 & 2.5 & 2.0 & 1.5 & 1.0 & 0.5 & 0.0\end{array}$

WQN-1718W

13C NMR in $\mathrm{CDCl} 3(100 \mathrm{MHz})$

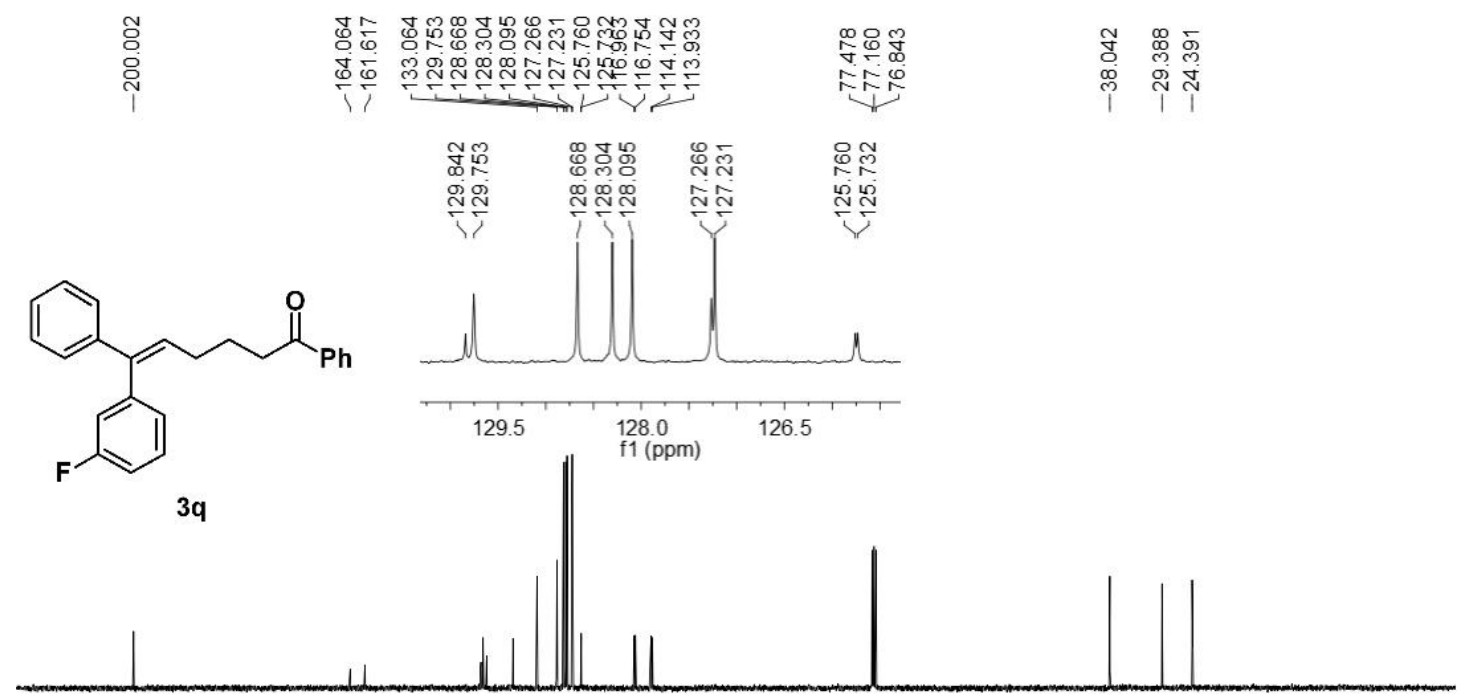

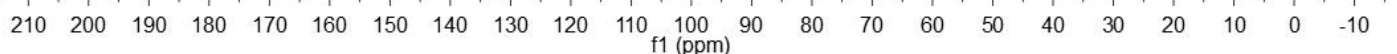


<smiles>O=C(CCCC=C(c1ccccc1)c1ccccc1)c1cccc(C(F)(F)F)c1</smiles>

$3 \mathbf{r}$

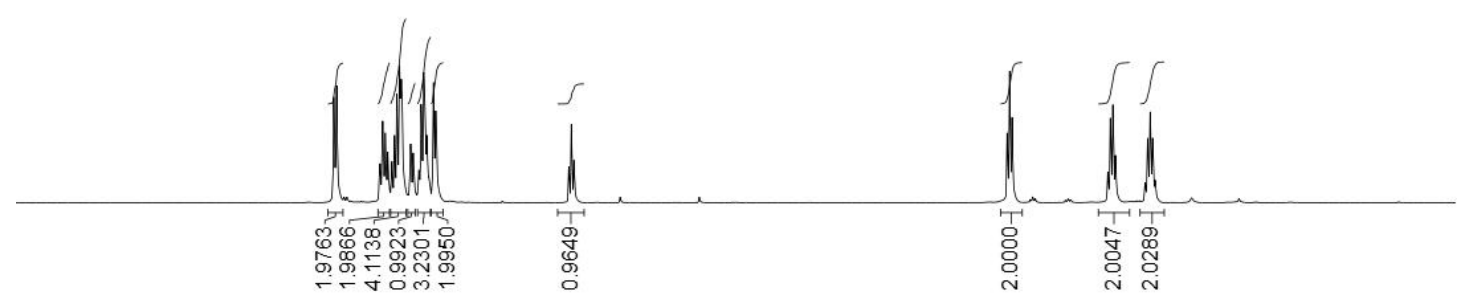

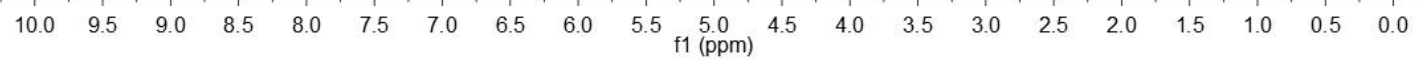

LJ-D535-P

LJ-D535 13C NMR in CDC13

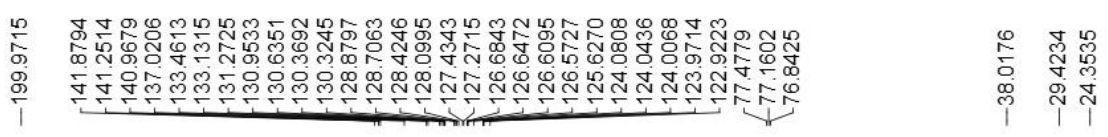<smiles>O=C(CCCC=C(c1ccccc1)c1ccccc1)c1ccccc1</smiles>

$3 \mathbf{r}$



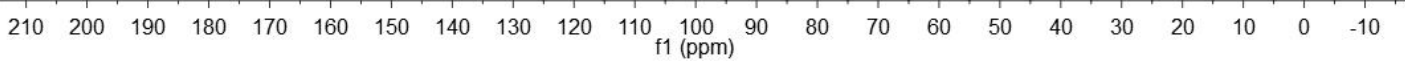


WQN-1660W

PROTON CDC13 \{D: \NMR400\203\} nmr 33

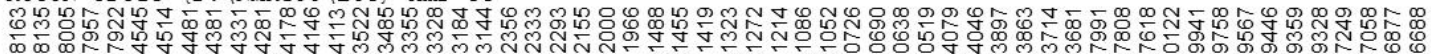<smiles>C/C(=C/CCCC(=O)c1ccccc1)c1ccccc1</smiles>

$3 s(3 s: 4 s=10: 1)$

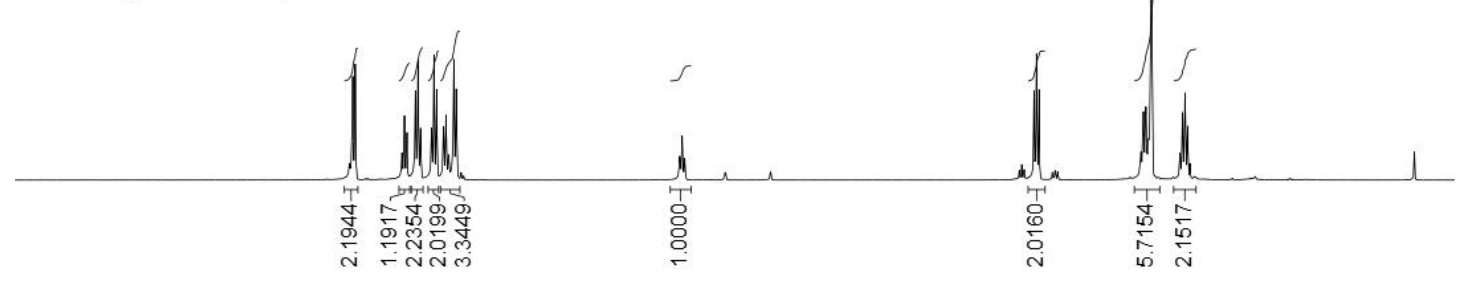

$\begin{array}{lllllllllllllllllllll}10.0 & 9.5 & 9.0 & 8.5 & 8.0 & 7.5 & 7.0 & 6.5 & 6.0 & 5.5 & \begin{array}{l}5.0 \\ \mathrm{f} 1(\mathrm{ppm})\end{array} & 4.5 & 4.0 & 3.5 & 3.0 & 2.5 & 2.0 & 1.5 & 1.0 & 0.5 & 0.0\end{array}$

WQN-1660W

C13CPD CDC13 \{D: \NMR400\203\} nmr 33<smiles>C/C(=C/CCCC(=O)c1ccccc1)c1ccccc1</smiles>

$3 s(3 s: 4 s=10: 1)$

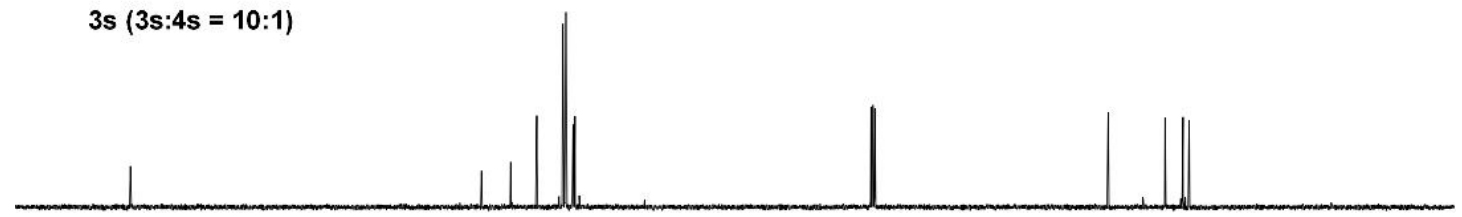

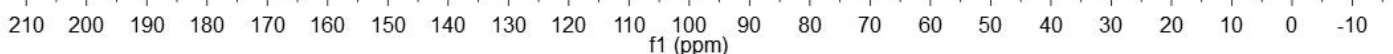


LJ-WQN-1

$1 \mathrm{H}$ NMR in $\mathrm{CDCl} 3(400 \mathrm{MHz})$

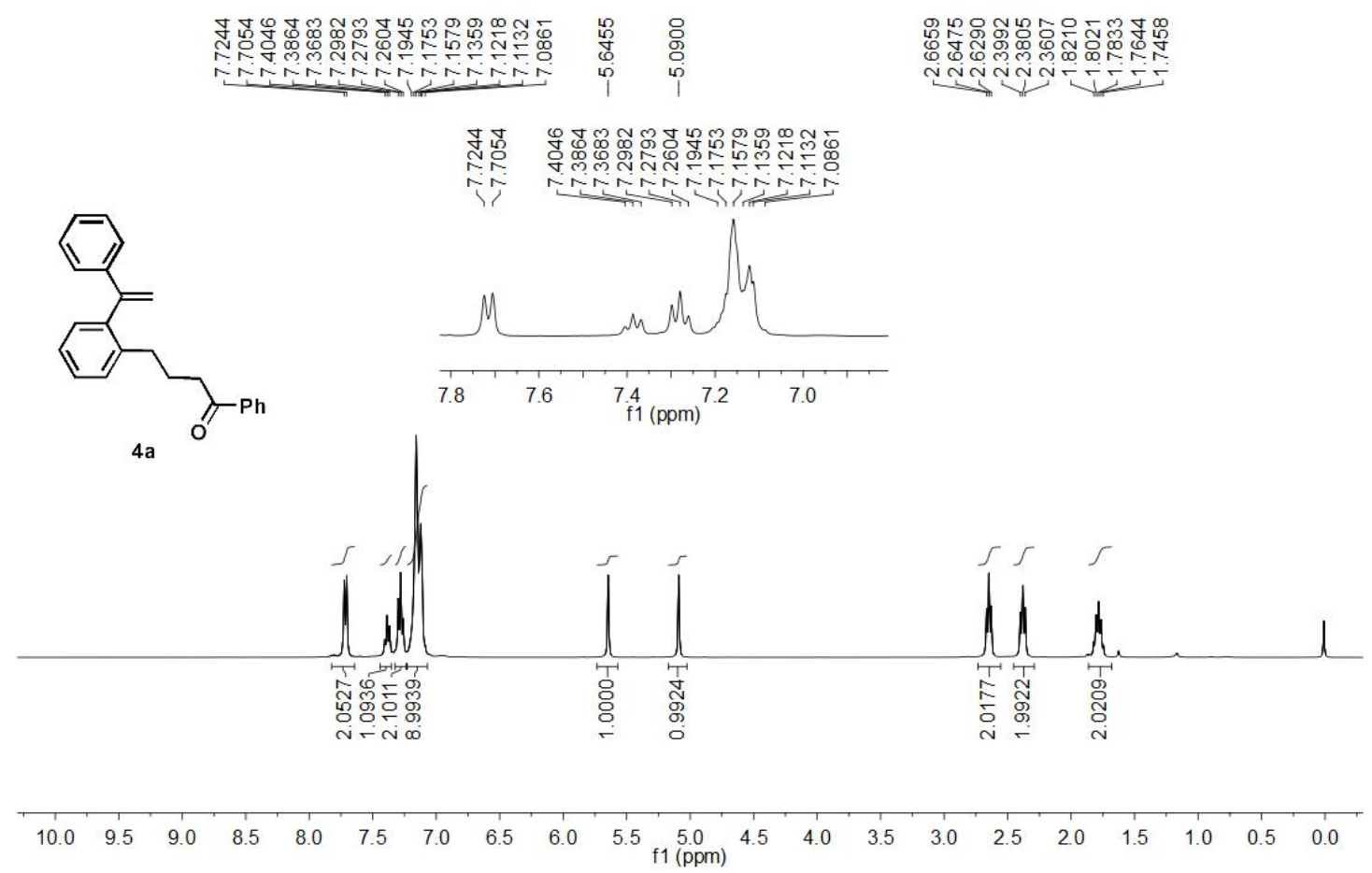

LJ-WQN-1

$13 \mathrm{C}$ NMR in $\mathrm{CDCl} 3(100 \mathrm{MHz})$
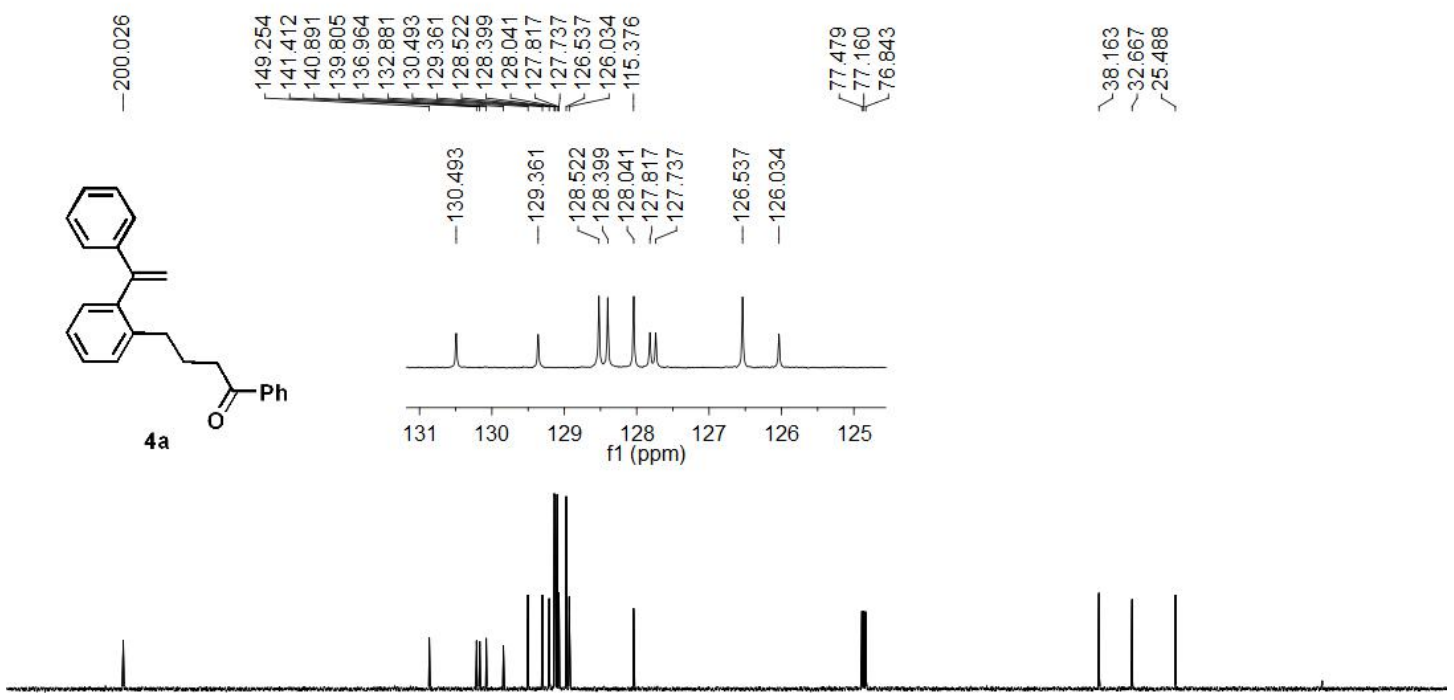

$\begin{array}{llllllllllllllllllllll}210 & 200 & 190 & 180 & 170 & 160 & 150 & 140 & 130 & 120 & 110 \begin{array}{l}100 \\ \mathrm{f} 1(\mathrm{ppm})\end{array} & 90 & 80 & 70 & 60 & 50 & 40 & 30 & 20 & 10 & 0 & -10\end{array}$ 

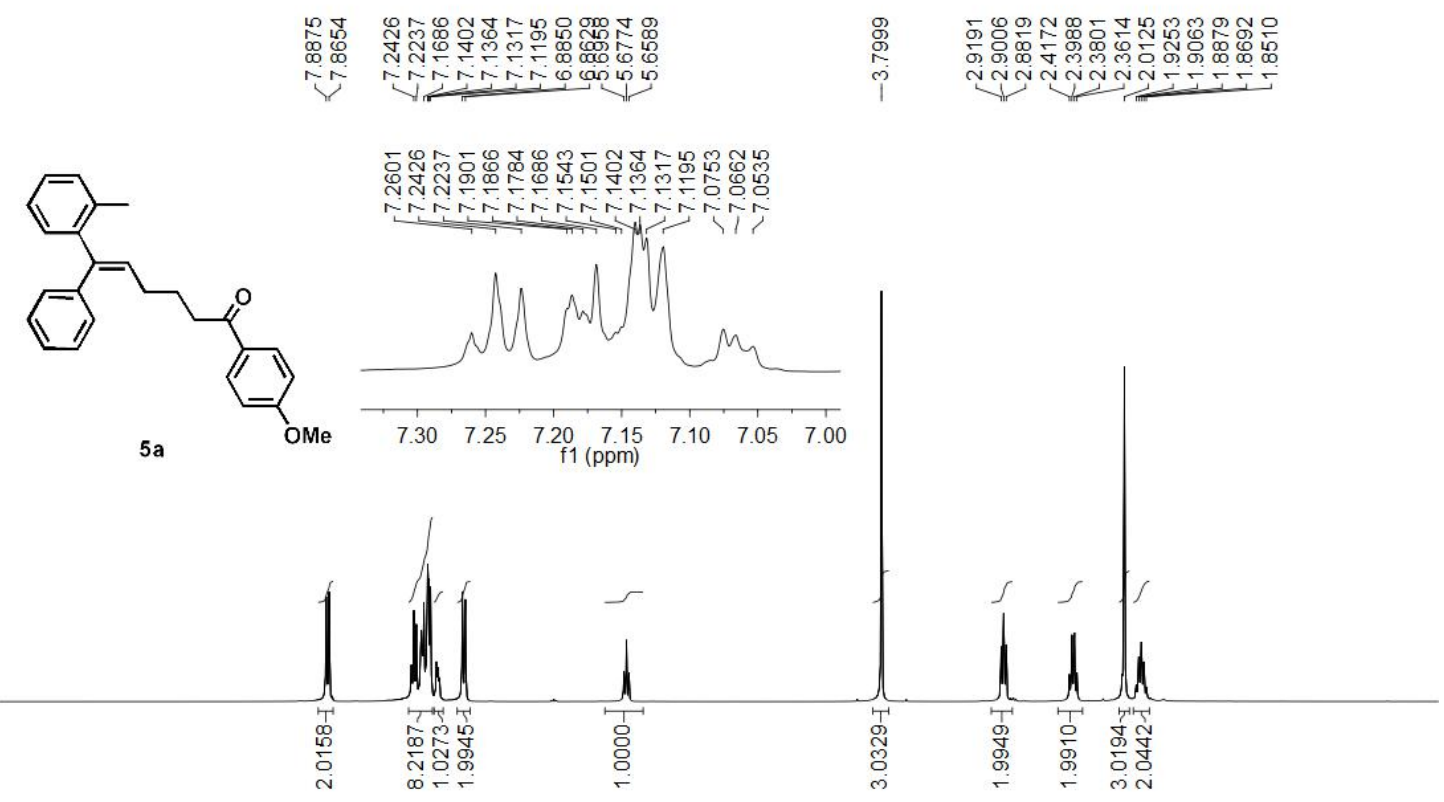

$\begin{array}{lllllllllllllllllllll}10.0 & 9.5 & 9.0 & 8.5 & 8.0 & 7.5 & 7.0 & 6.5 & 6.0 & 5.5 & \begin{array}{c}5.0 \\ \mathrm{fpm})\end{array} & 4.5 & 4.0 & 3.5 & 3.0 & 2.5 & 2.0 & 1.5 & 1.0 & 0.5 & 0.0\end{array}$

WQN-1699W

13C NMR in $\mathrm{CDCl} 3(100 \mathrm{MHz})$
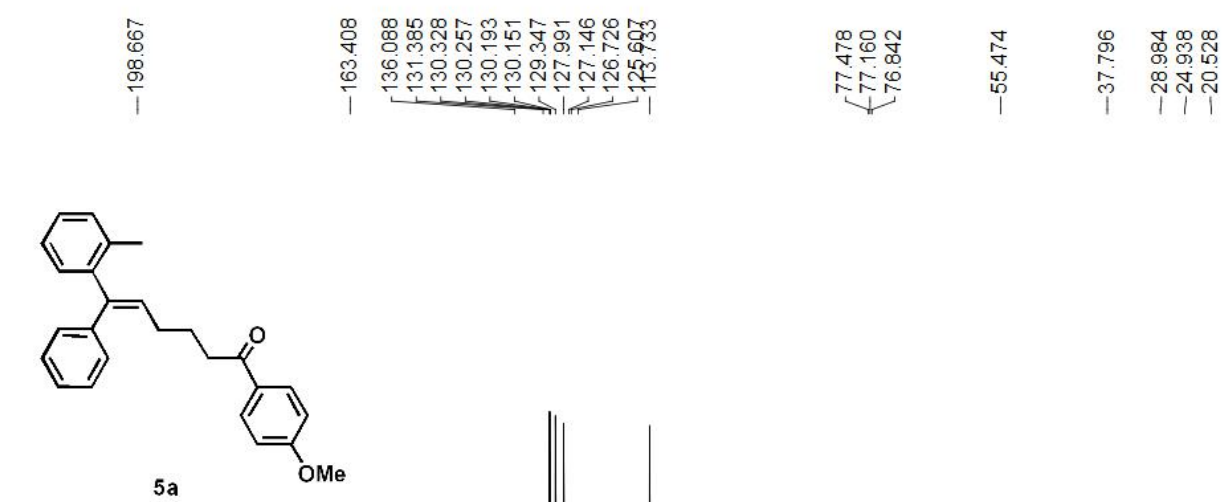

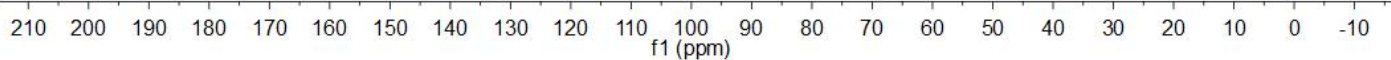


WQN-1700W

1H NMR in $\mathrm{CDCl} 3(400 \mathrm{MHz})$

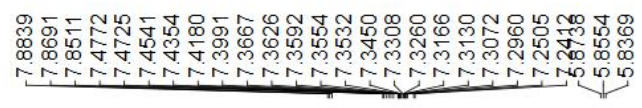

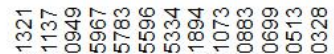 \\ กั่งก}

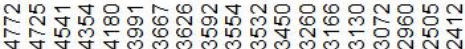
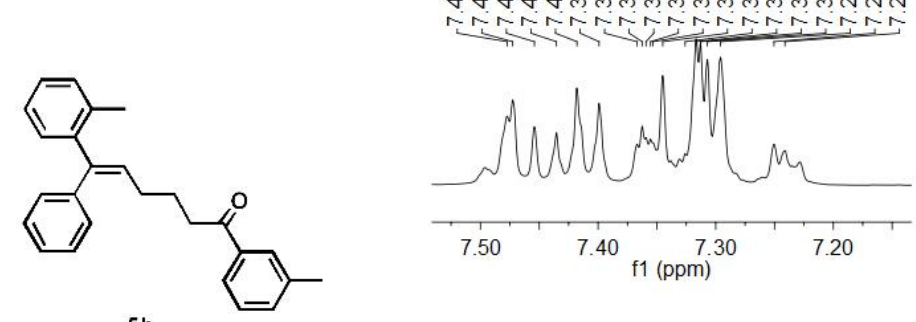

$5 b$

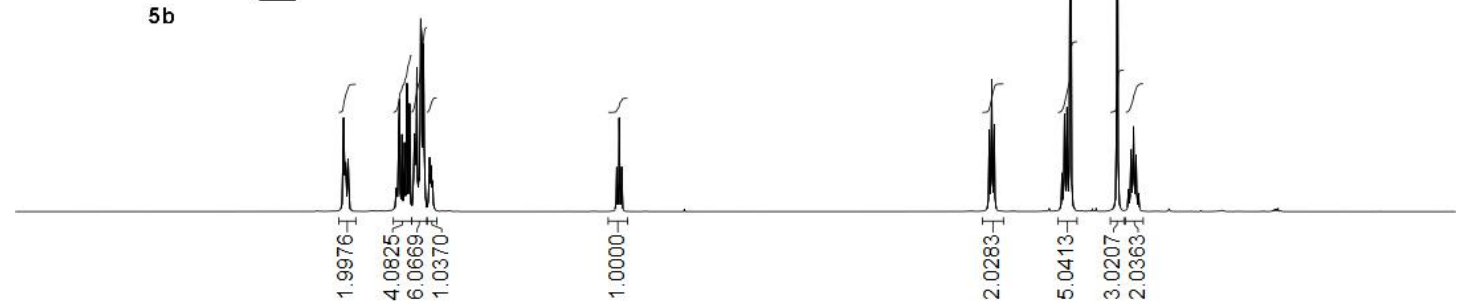

$\begin{array}{lllllllllllllllllllll}10.0 & 9.5 & 9.0 & 8.5 & 8.0 & 7.5 & 7.0 & 6.5 & 6.0 & 5.5 & \begin{array}{c}5.0 \\ \mathrm{f} 1(\mathrm{ppm})\end{array} & 4.5 & 4.0 & 3.5 & 3.0 & 2.5 & 2.0 & 1.5 & 1.0 & 0.5 & 0.0\end{array}$

WQN-1700W

13C NMR in $\mathrm{CDCl} 3(100 \mathrm{MHz})$
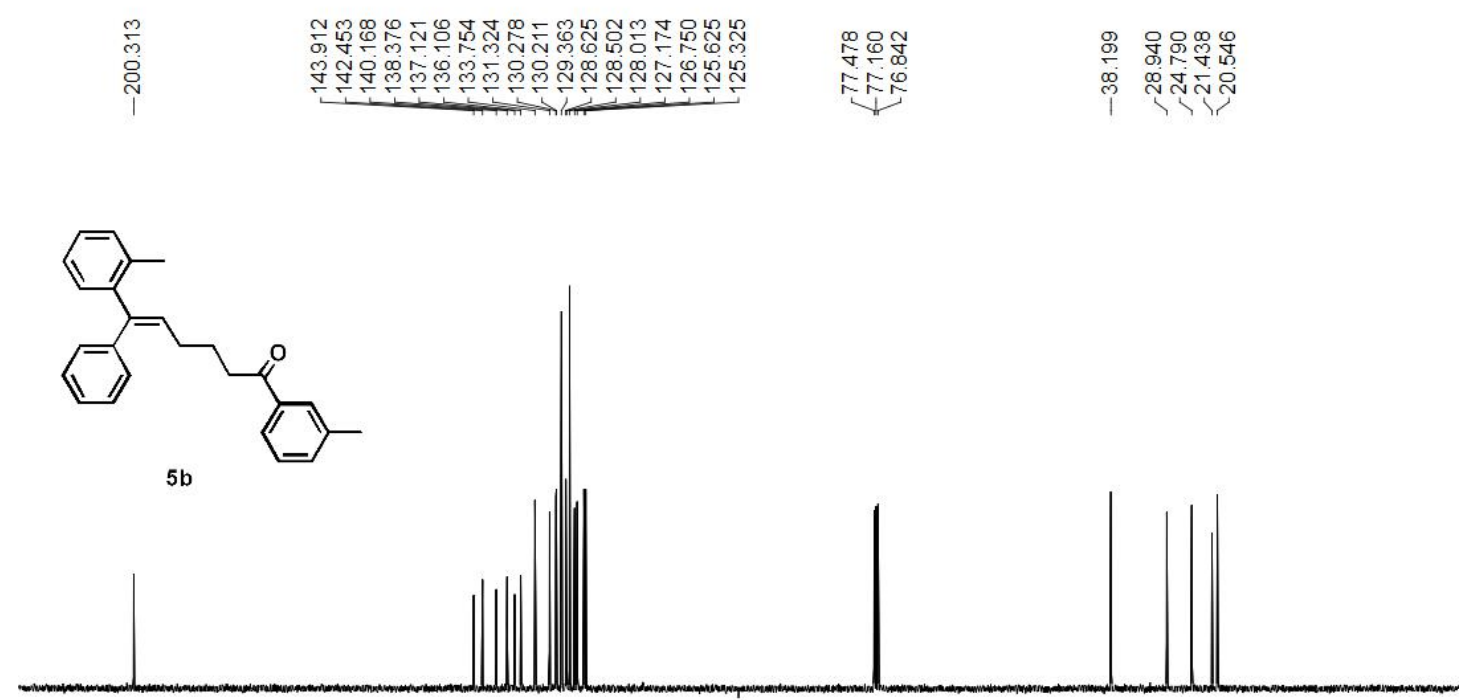

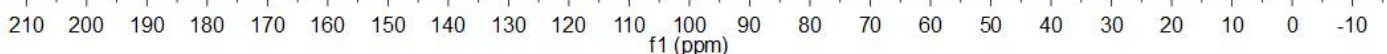


WQN-1707W

1H NMR in $\mathrm{CDCl} 3(400 \mathrm{MHz})$

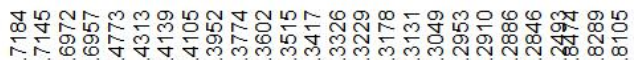

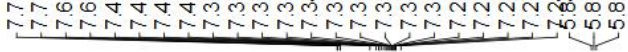
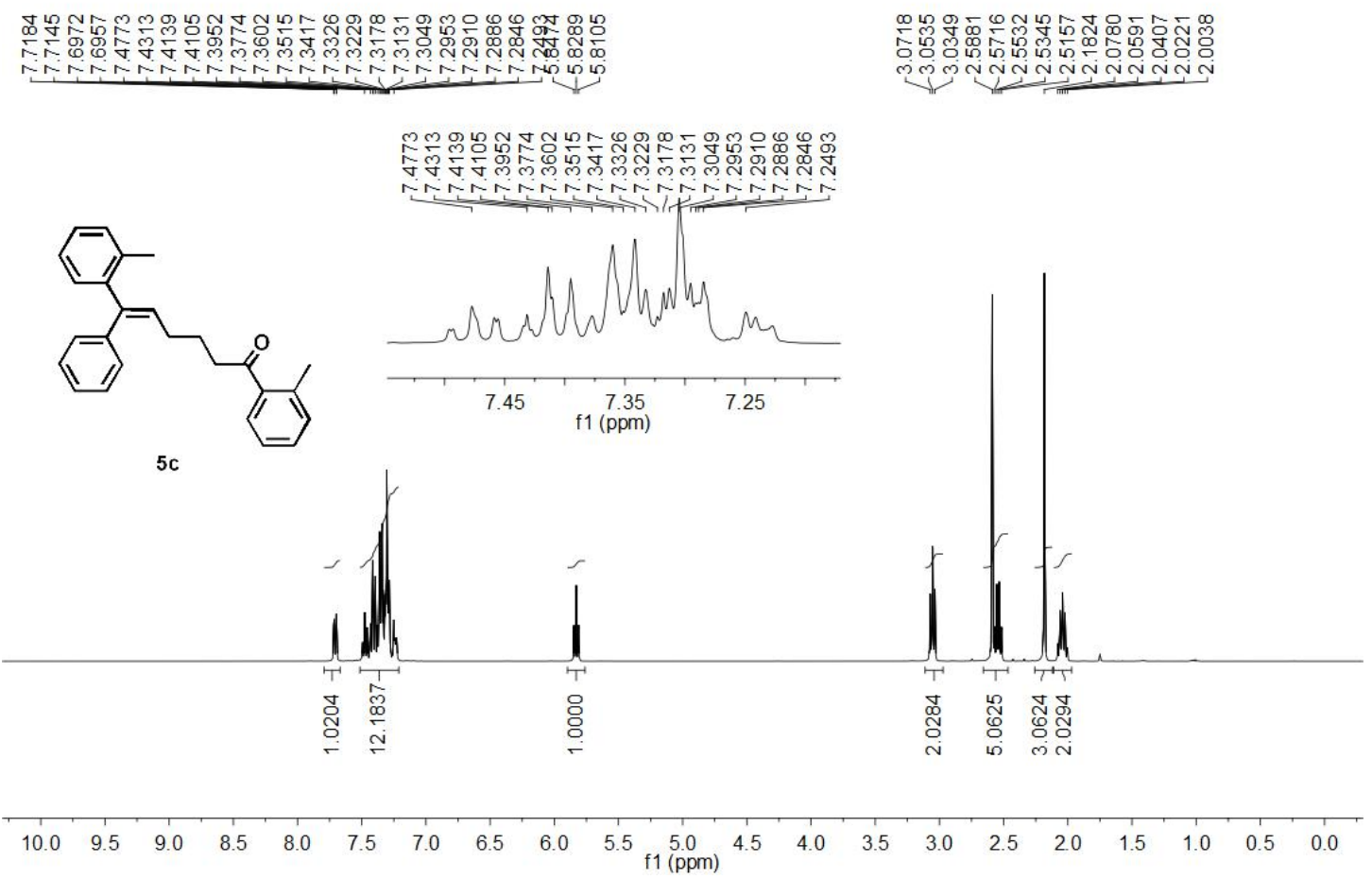

WQN-1707W

13C NMR in $\mathrm{CDCl} 3(100 \mathrm{MHz})$
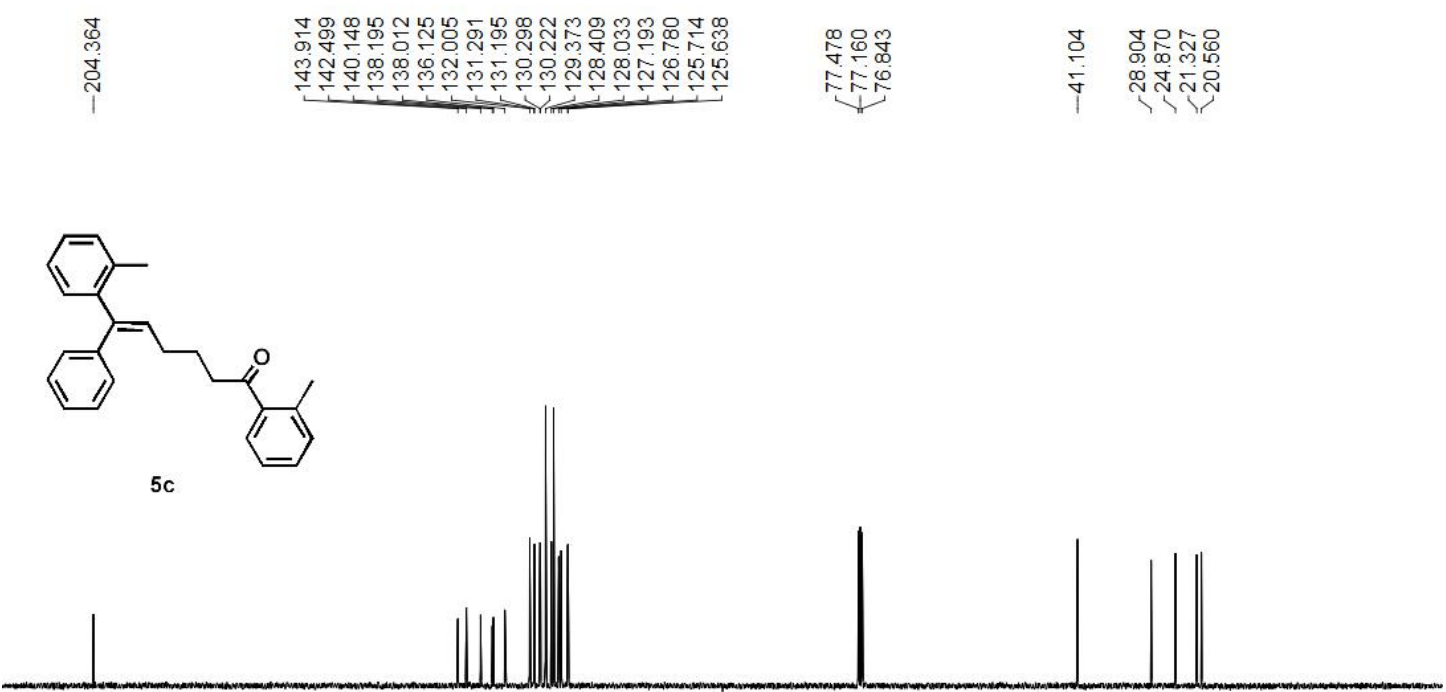

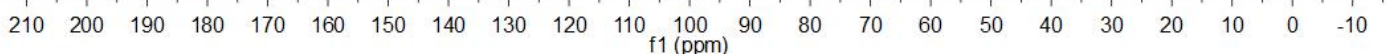


WQN-1715W

$1 \mathrm{H}$ NMR in $\mathrm{CDCl} 3(400 \mathrm{MHz})$

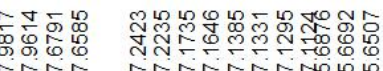

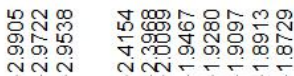

rivininingus
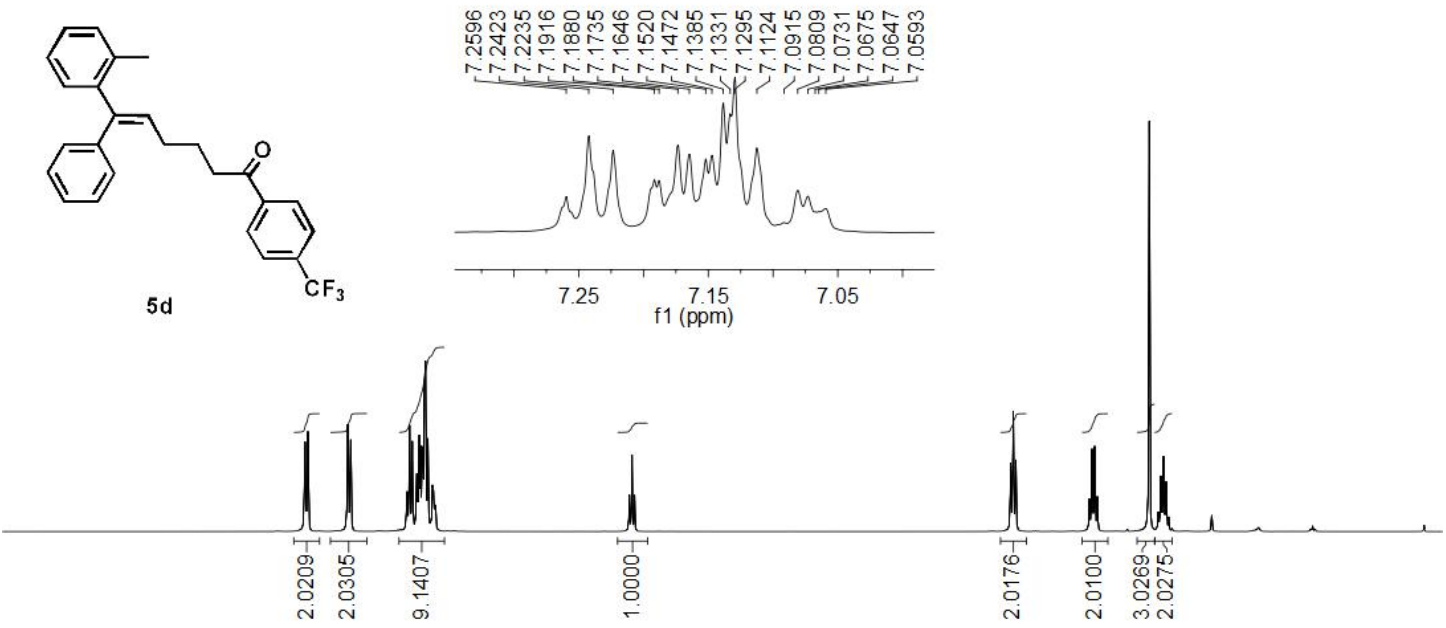

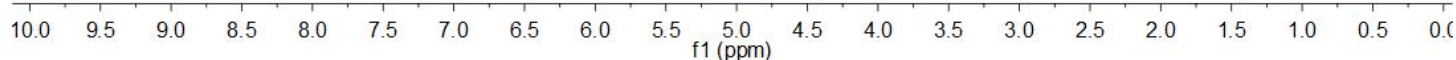

WQN-1715W

13C NMR in $\operatorname{CDCl} 3(100 \mathrm{MHz})$

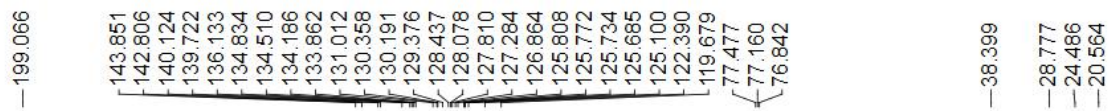
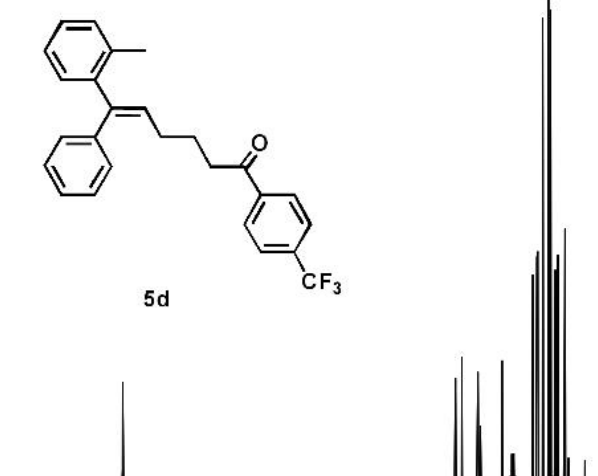

$\begin{array}{lllllllllllllllllllllll}210 & 200 & 190 & 180 & 170 & 160 & 150 & 140 & 130 & 120 & 110 & 100 & 90 & 80 & 70 & 60 & 50 & 40 & 30 & 20 & 10 & 0 & -10\end{array}$ 


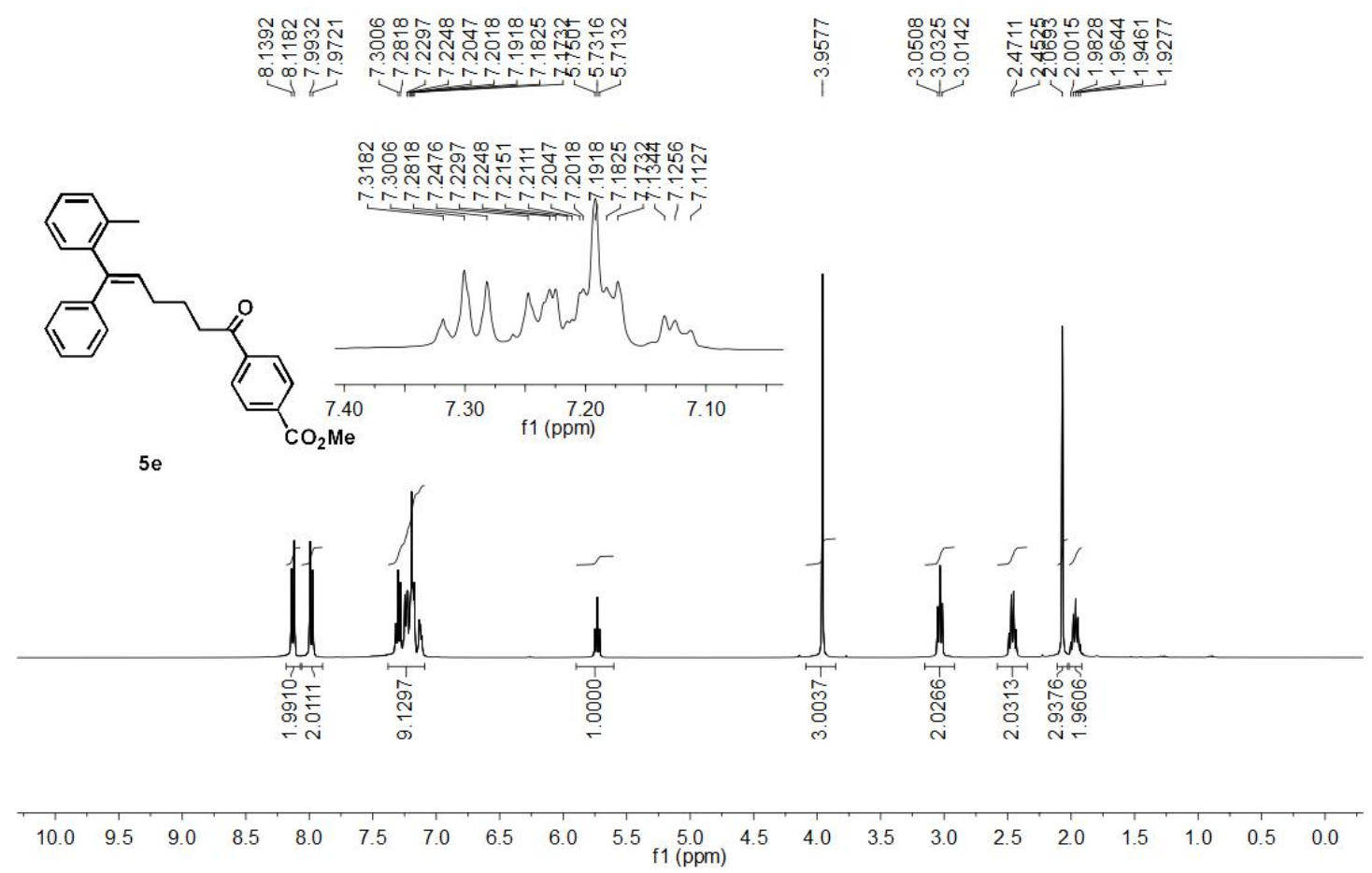

WQN-1725W

13C NMR in $\operatorname{CDCl3}(100 \mathrm{MHz})$

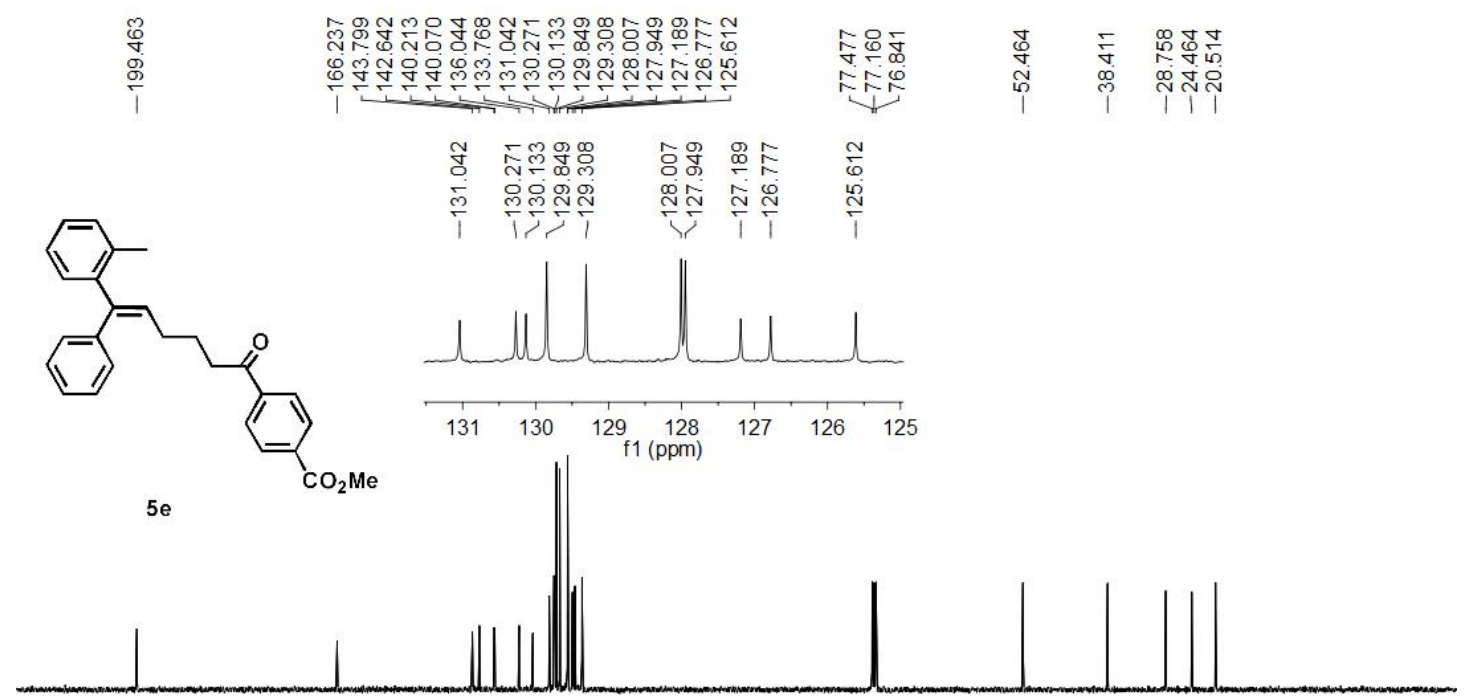

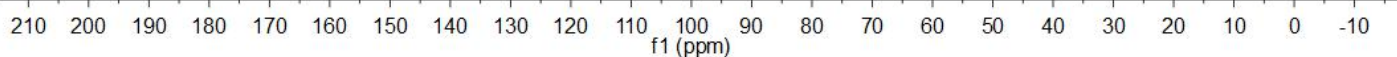



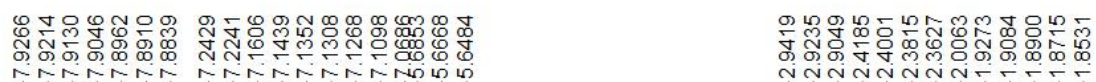

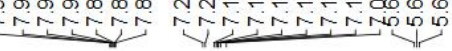

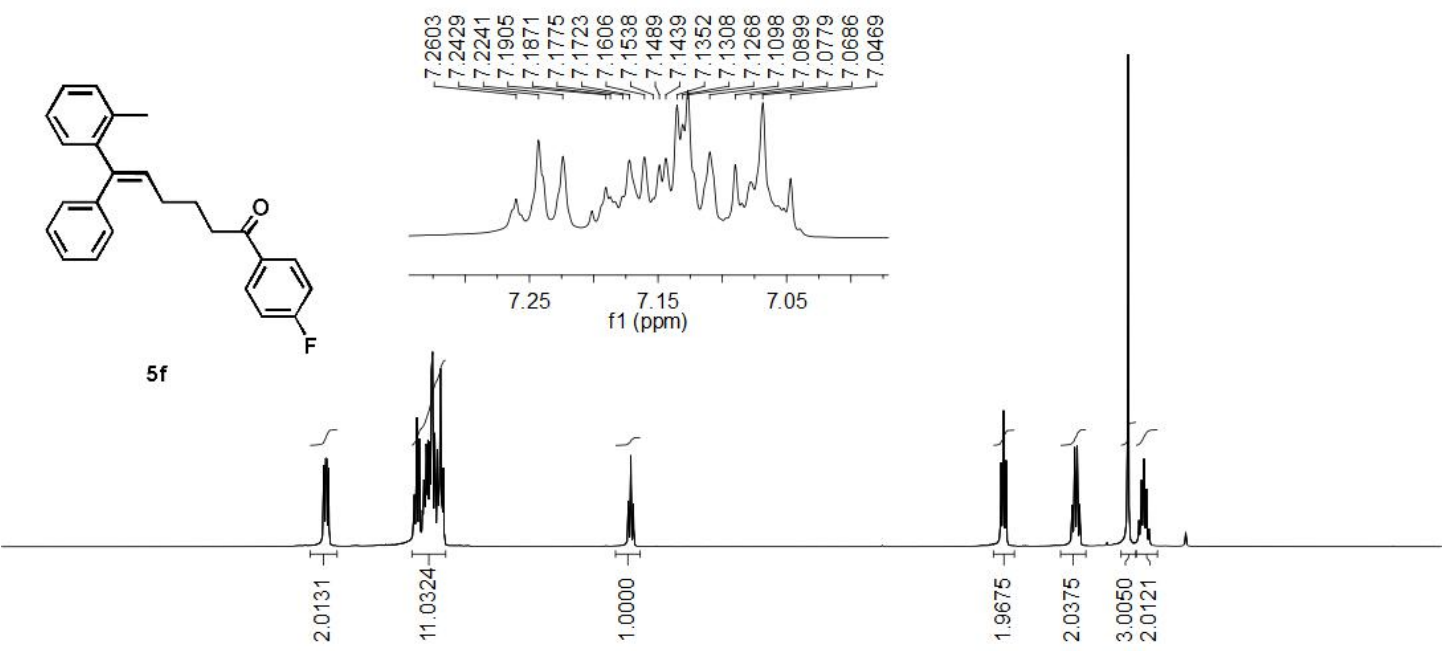

$\begin{array}{llllllllllllllllllllll}10.0 & 9.5 & 9.0 & 8.5 & 8.0 & 7.5 & 7.0 & 6.5 & 6.0 & 5.5 & \begin{array}{c}1.0 \\ \mathrm{f} 1(\mathrm{ppm})\end{array} & 4.5 & 4.0 & 3.5 & 3.0 & 2.5 & 2.0 & 1.5 & 1.0 & 0.5 & 0.0\end{array}$

WQN-1709W

13C NMR in $\mathrm{CDCl} 3(100 \mathrm{MHz})$

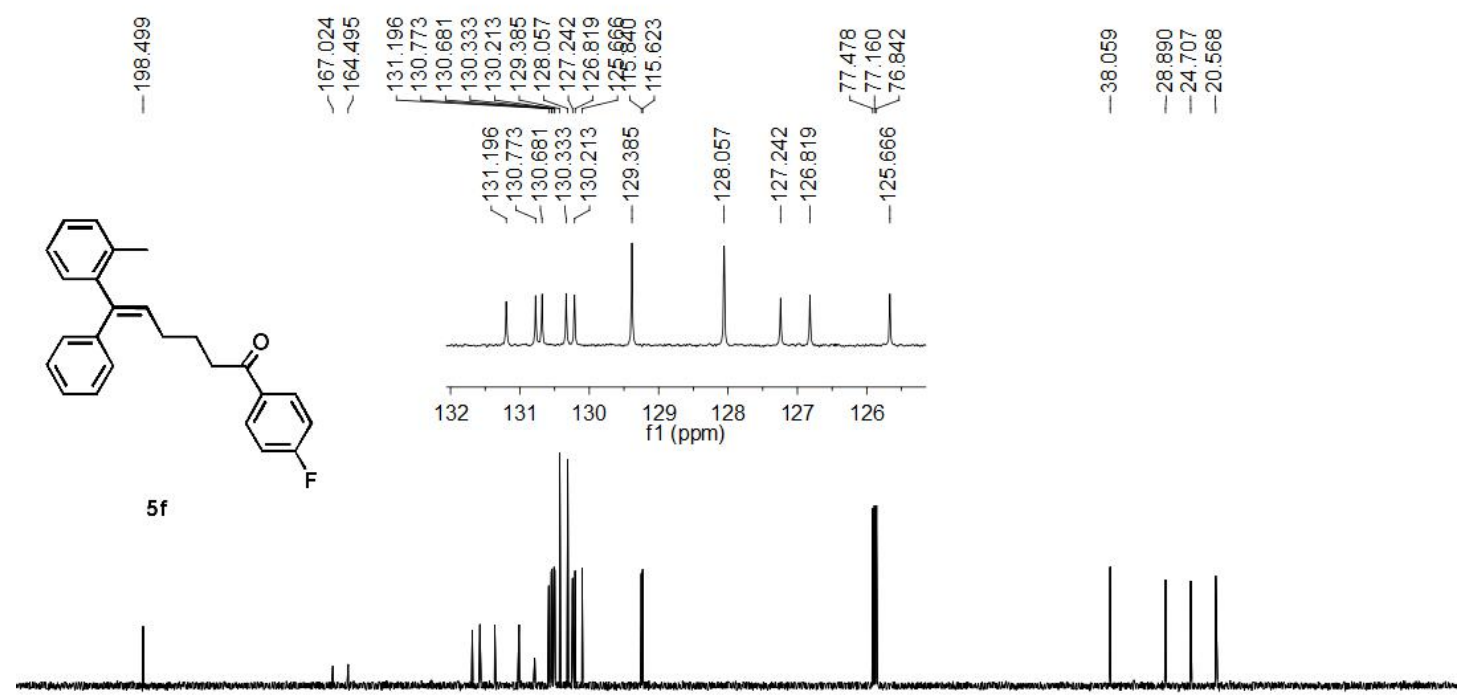

$\begin{array}{llllllllllllllllllllll}210 & 200 & 190 & 180 & 170 & 160 & 150 & 140 & 130 & 120 & 110 \begin{array}{l}100 \\ \mathrm{f} 1(\mathrm{ppm})\end{array} & 90 & 80 & 70 & 60 & 50 & 40 & 30 & 20 & 10 & 0 & -10\end{array}$ 
WQN-1712W

1H NMR in $\mathrm{CDCl} 3(400 \mathrm{MHz})$

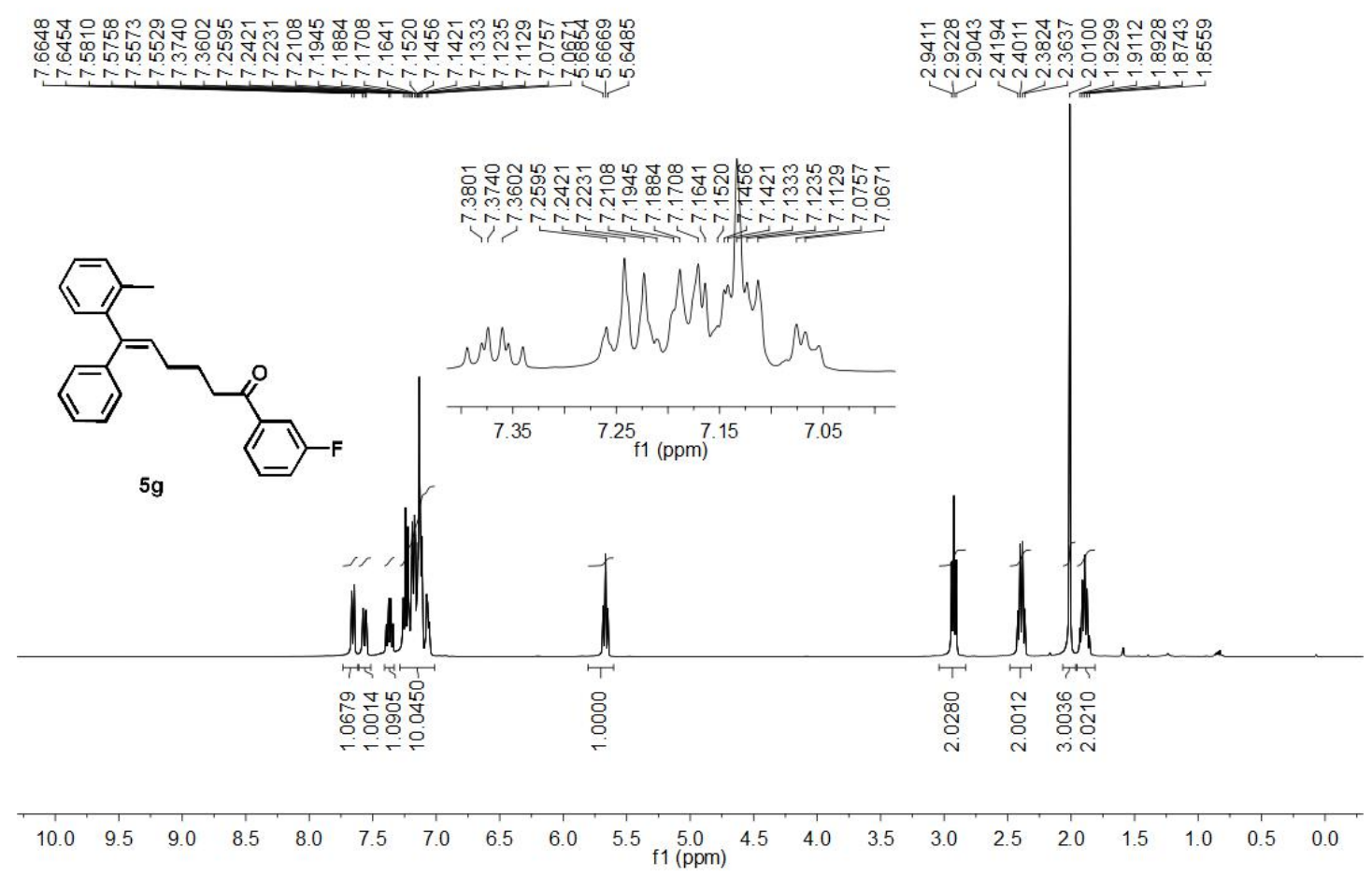

WQN-1712W

$13 \mathrm{C}$ NMR in $\mathrm{CDCl} 3(100 \mathrm{MHz})$
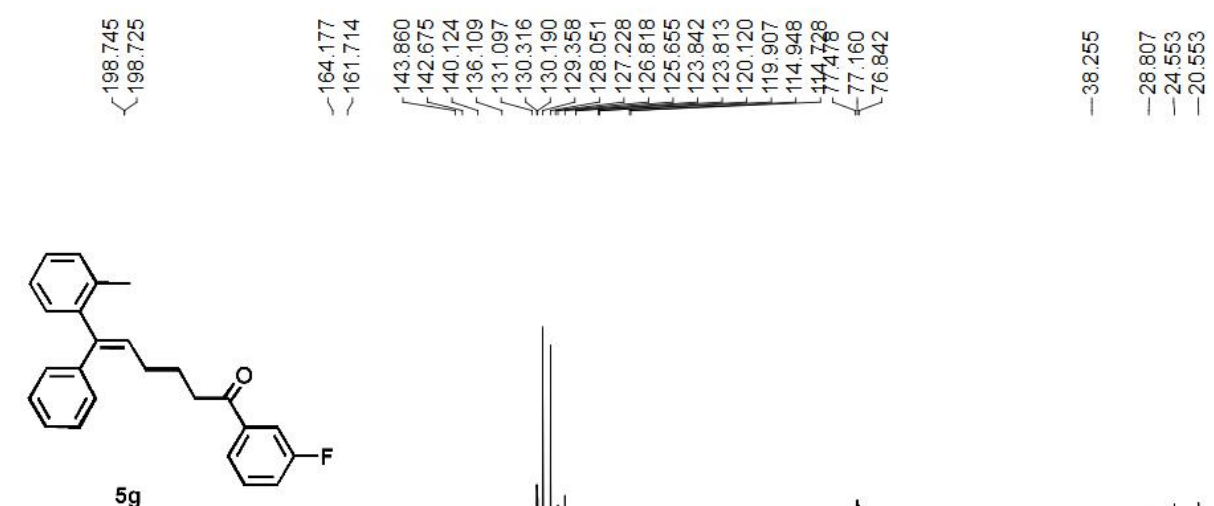

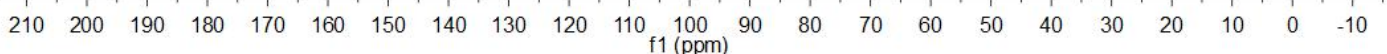




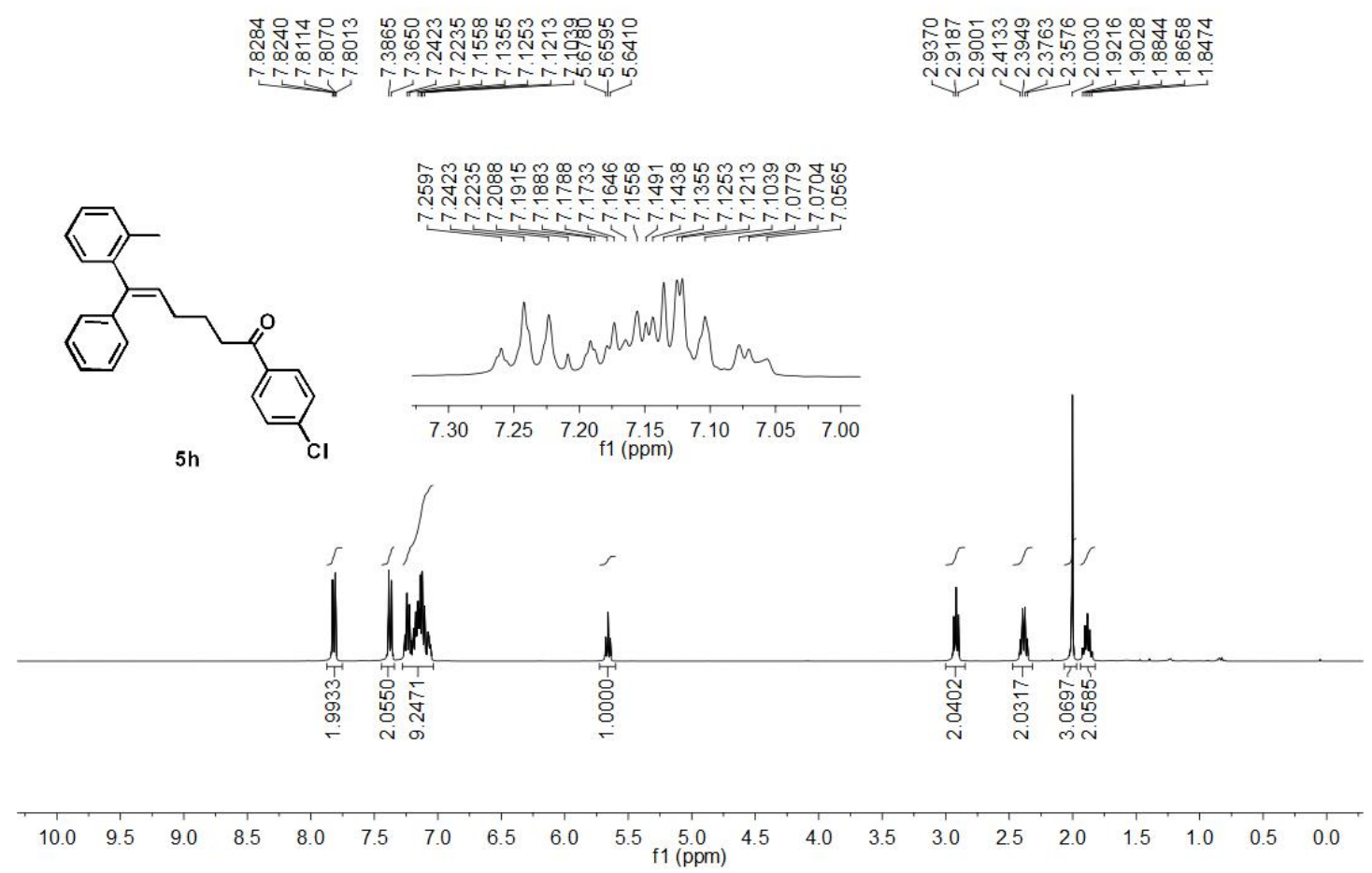

WQN-1719W

13C NMR in $\mathrm{CDCl} 3(100 \mathrm{MHz})$

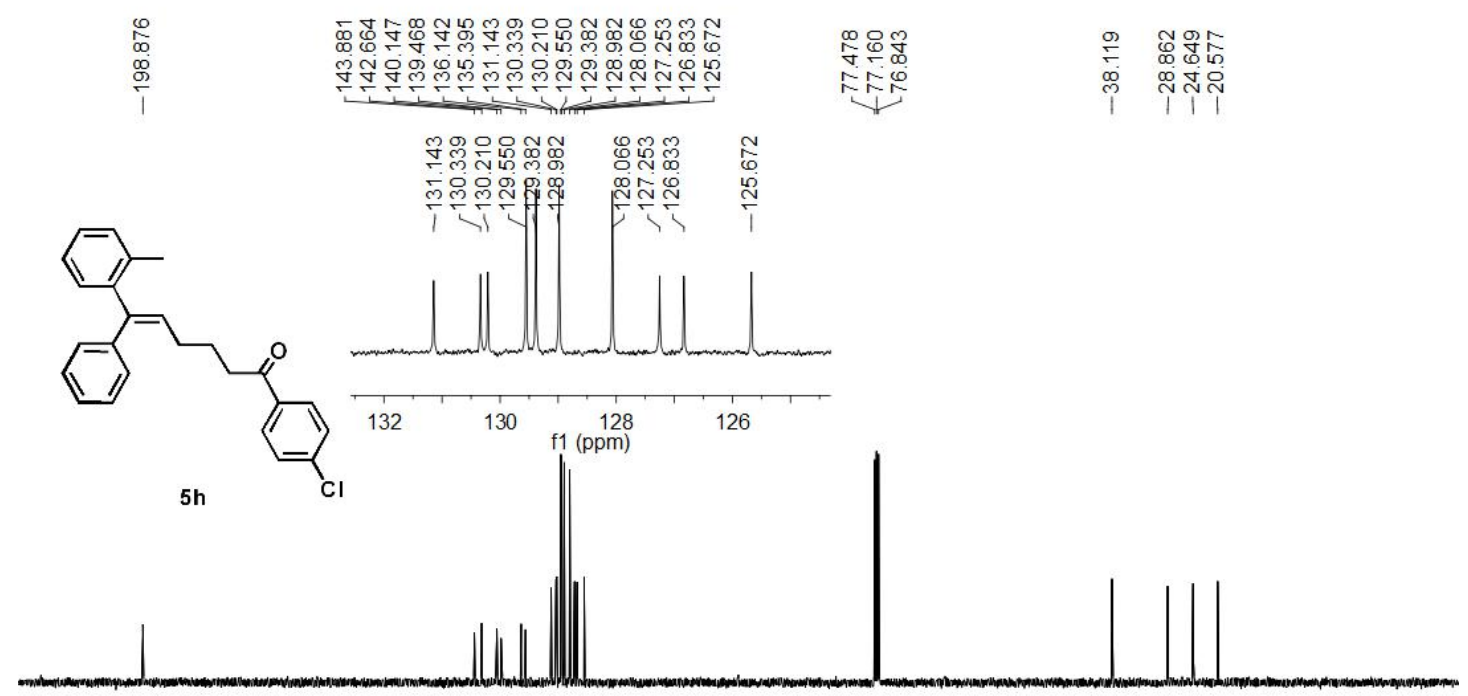

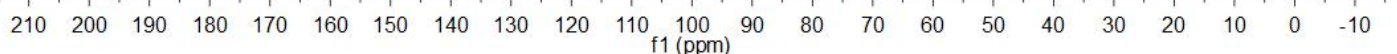



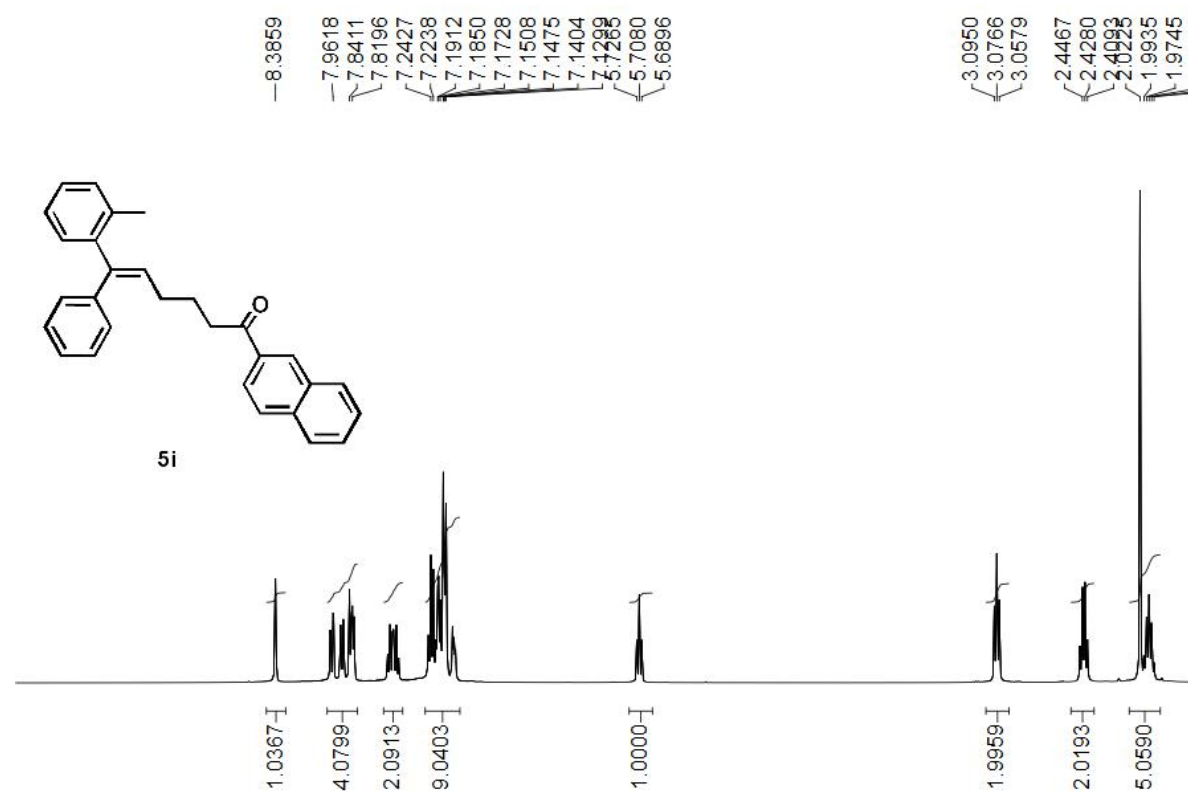

$\begin{array}{lllllllllllllllllllll}10.0 & 9.5 & 9.0 & 8.5 & 8.0 & 7.5 & 7.0 & 6.5 & 6.0 & 5.5 & \begin{array}{c}5.0 \\ \mathrm{fpm})\end{array} & 4.5 & 4.0 & 3.5 & 3.0 & 2.5 & 2.0 & 1.5 & 1.0 & 0.5 & 0.0\end{array}$

WQN-1721W

13C NMR in $\mathrm{CDCl} 3(100 \mathrm{MHz})$

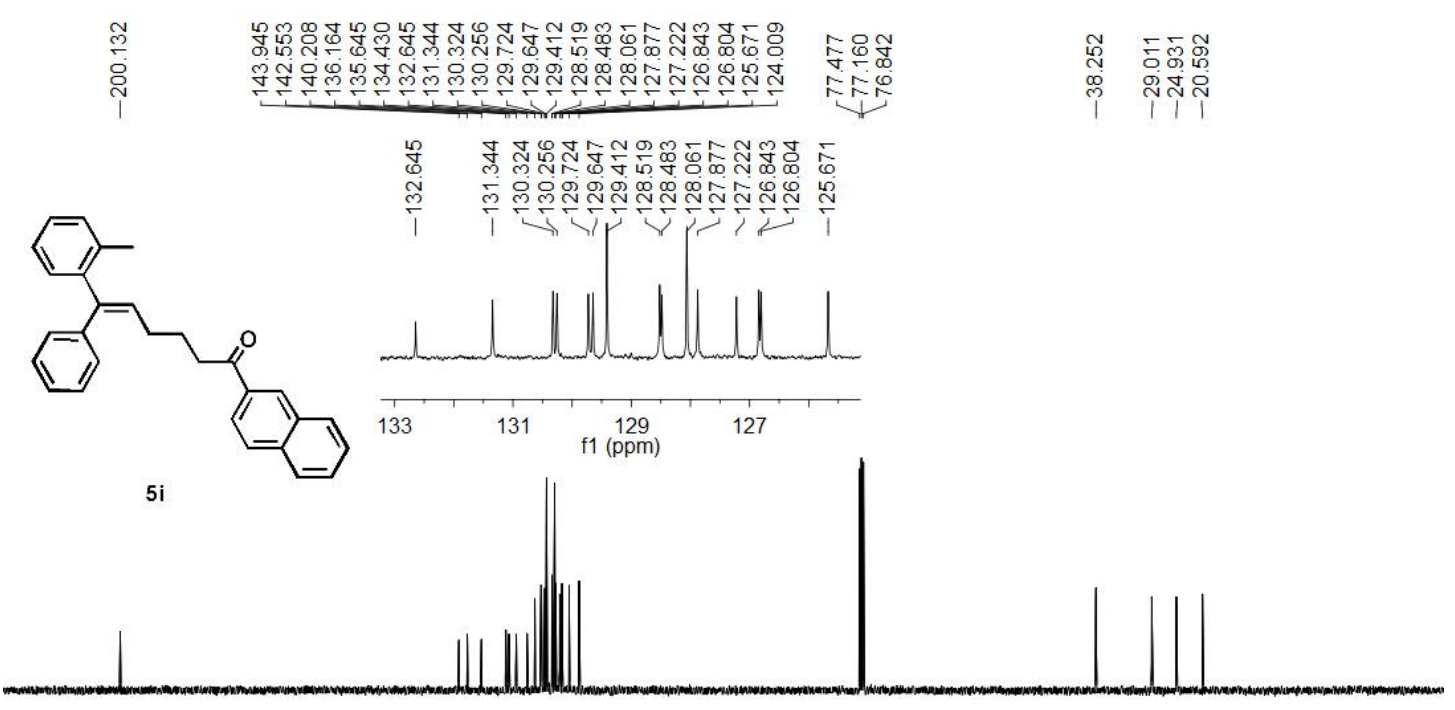

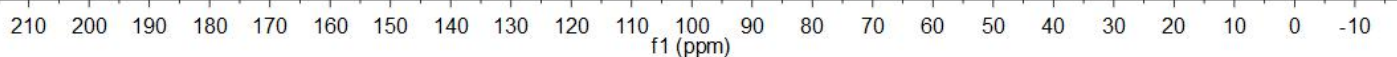


WQN-1723W

$1 \mathrm{H} \mathrm{NMR} \mathrm{in} \mathrm{CDCl} 3(400 \mathrm{MHz})$

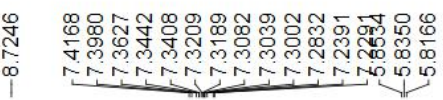
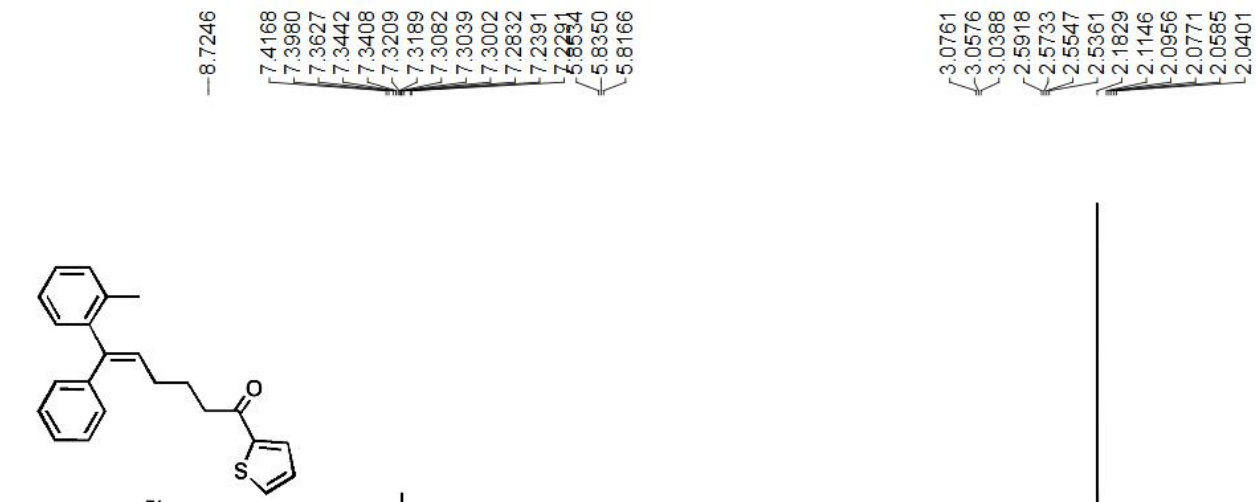

$5 \mathbf{j}$

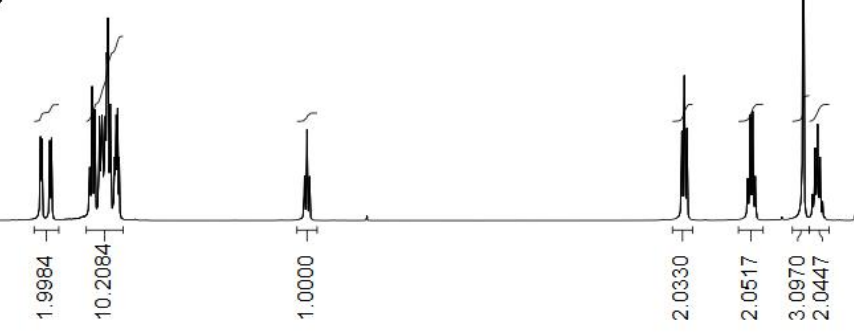

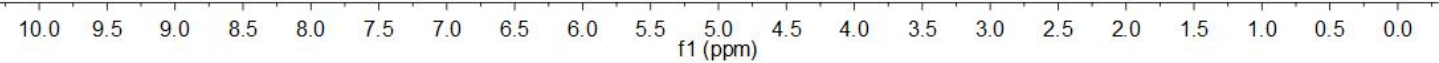

WQN-1723W

13C NMR in $\mathrm{CDCl3}(100 \mathrm{MHz})$

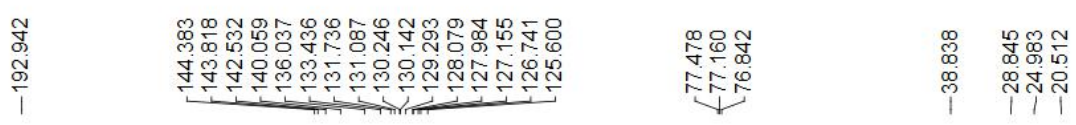

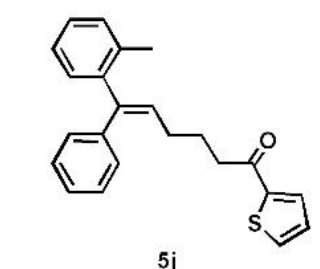

$5 j$

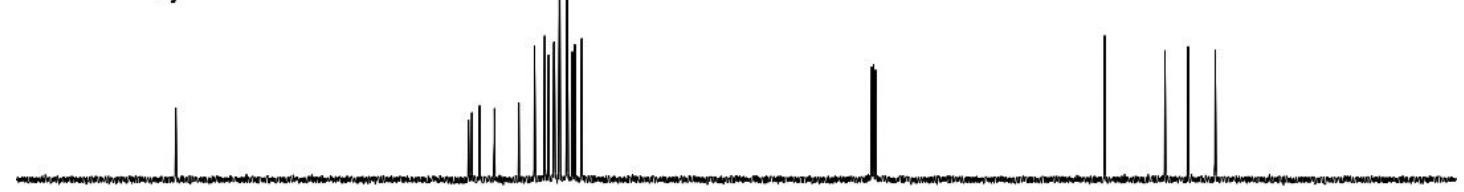

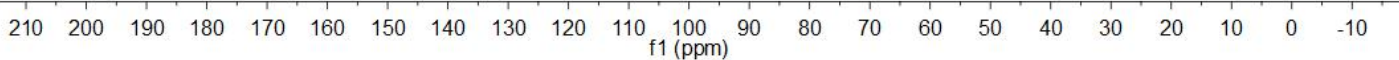


WQN-1726W

$1 \mathrm{H}$ NMR in $\mathrm{CDCl} 3(400 \mathrm{MHz})$
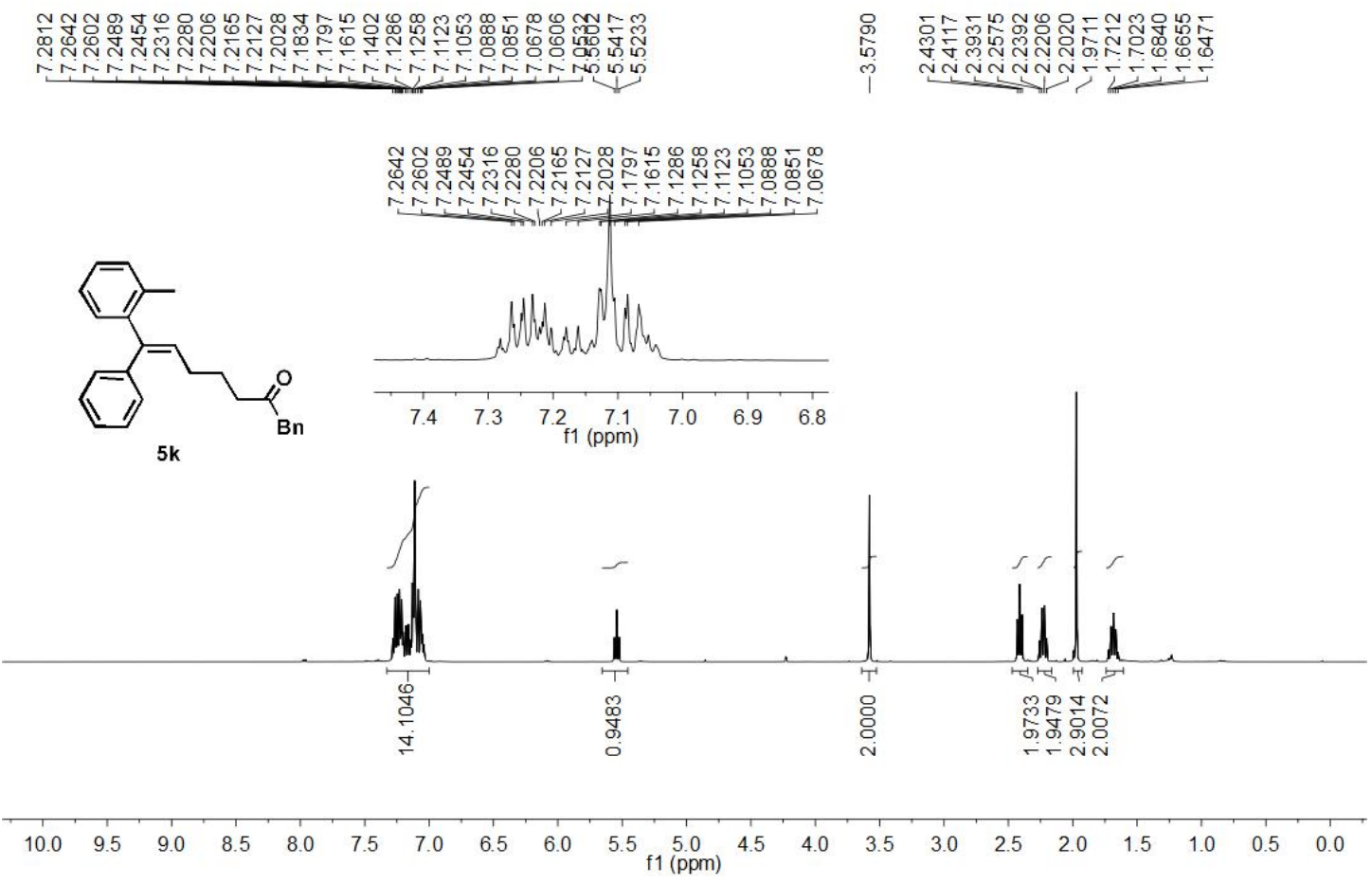

WQN-1726W

$13 \mathrm{C}$ NMR in $\mathrm{CDCl} 3(100 \mathrm{MHz})$



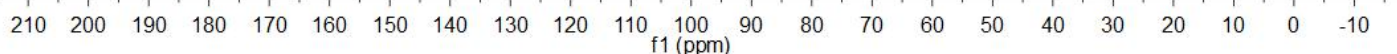


WQN-1753W

$1 \mathrm{H}$ NMR in $\mathrm{CDCl} 3(400 \mathrm{MHz})$

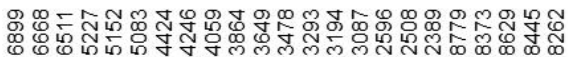

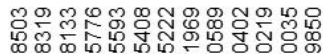

NกNกNก

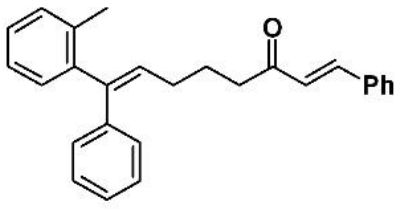

5I

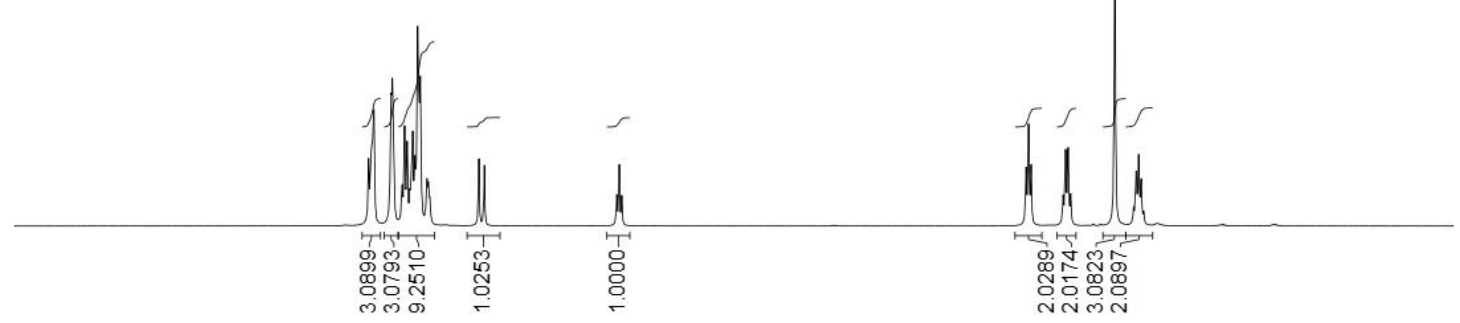

$\begin{array}{lllllllllllllllllllll}10.0 & 9.5 & 9.0 & 8.5 & 8.0 & 7.5 & 7.0 & 6.5 & 6.0 & 5.5 & \begin{array}{l}5.0 \\ \mathrm{f} 1(\mathrm{ppm})\end{array} & 4.5 & 4.0 & 3.5 & 3.0 & 2.5 & 2.0 & 1.5 & 1.0 & 0.5 & 0.0\end{array}$

WQN-1753W

13C NMR in $\mathrm{CDCl} 3(100 \mathrm{MHz})$

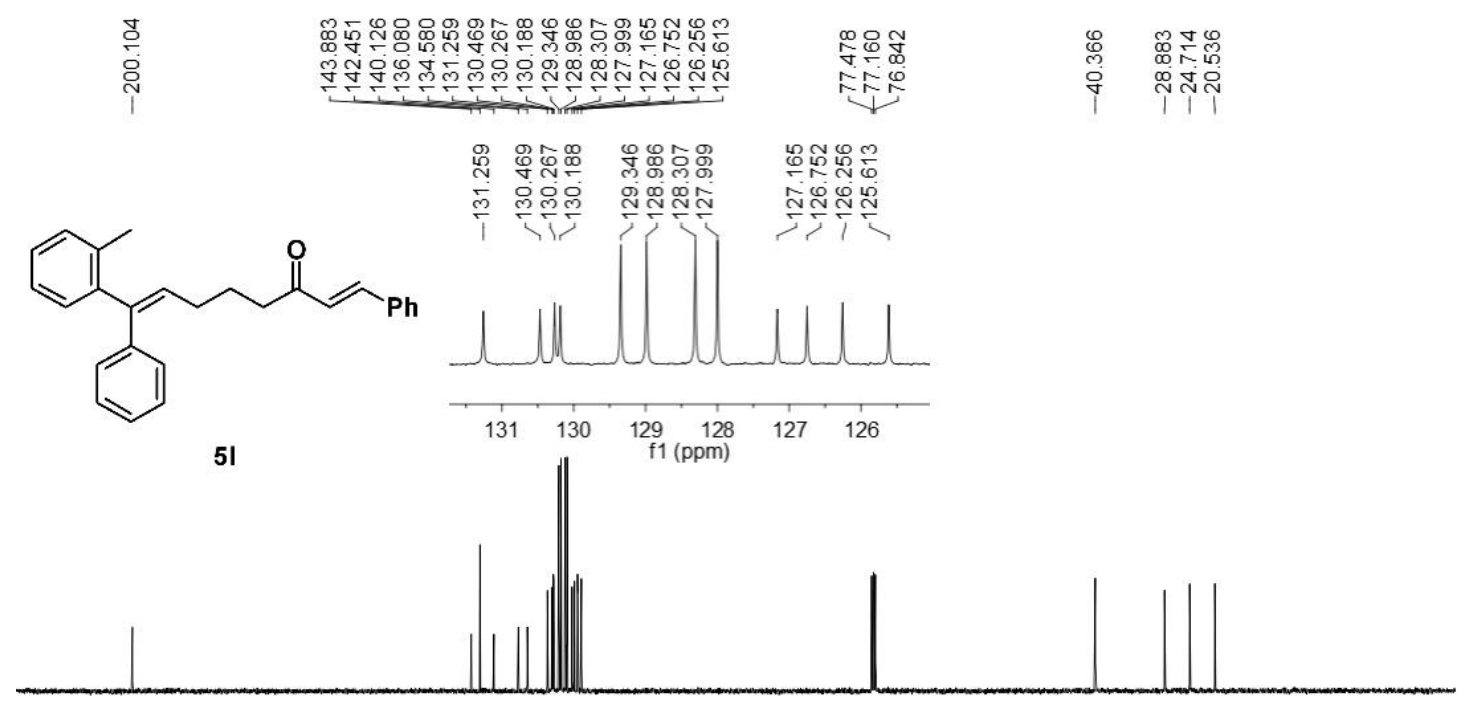

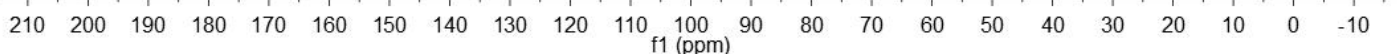




\section{以}

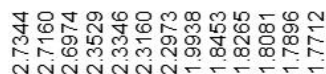

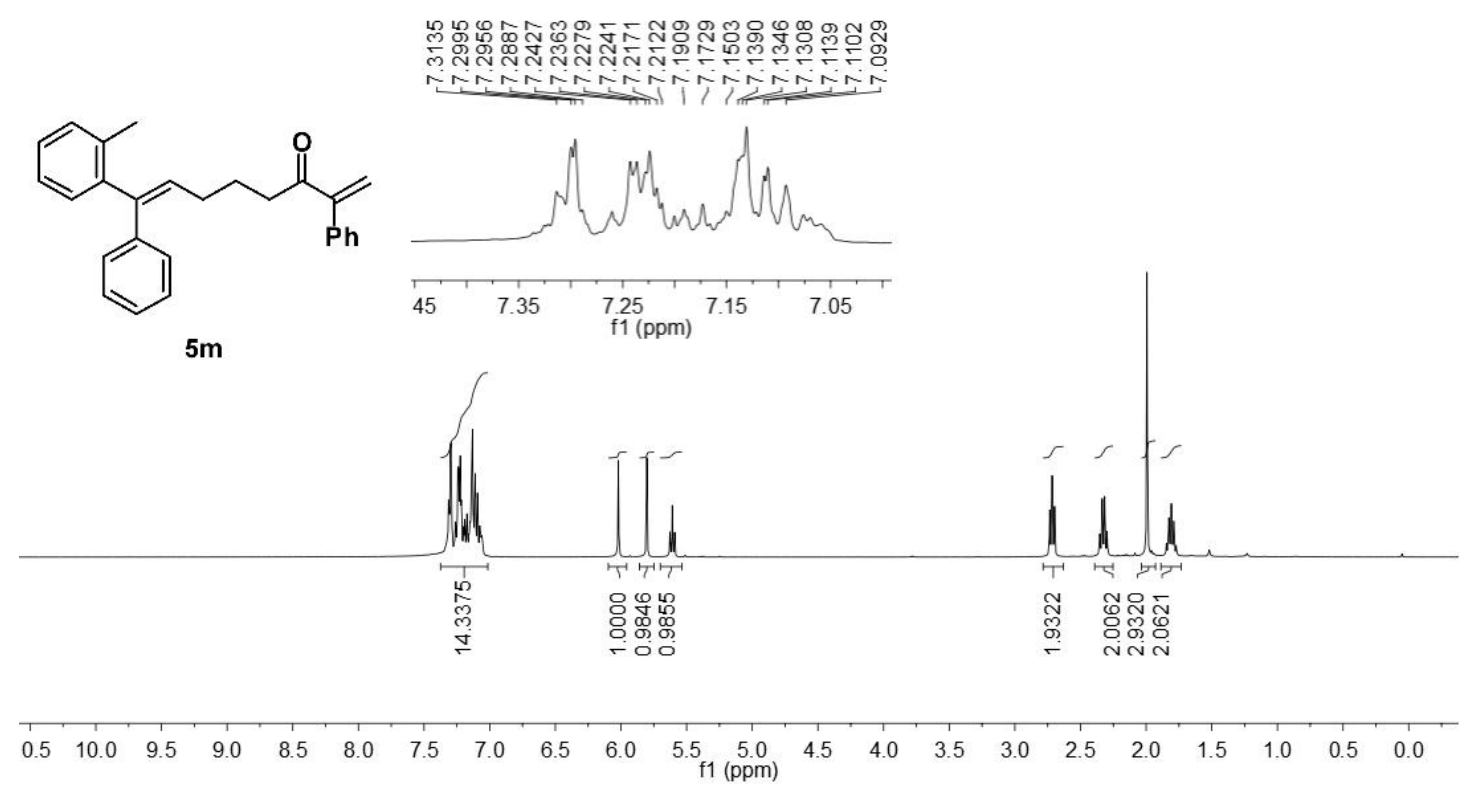

WQN-1741W

13C NMR in $\mathrm{CDCl} 3(100 \mathrm{MHz})$

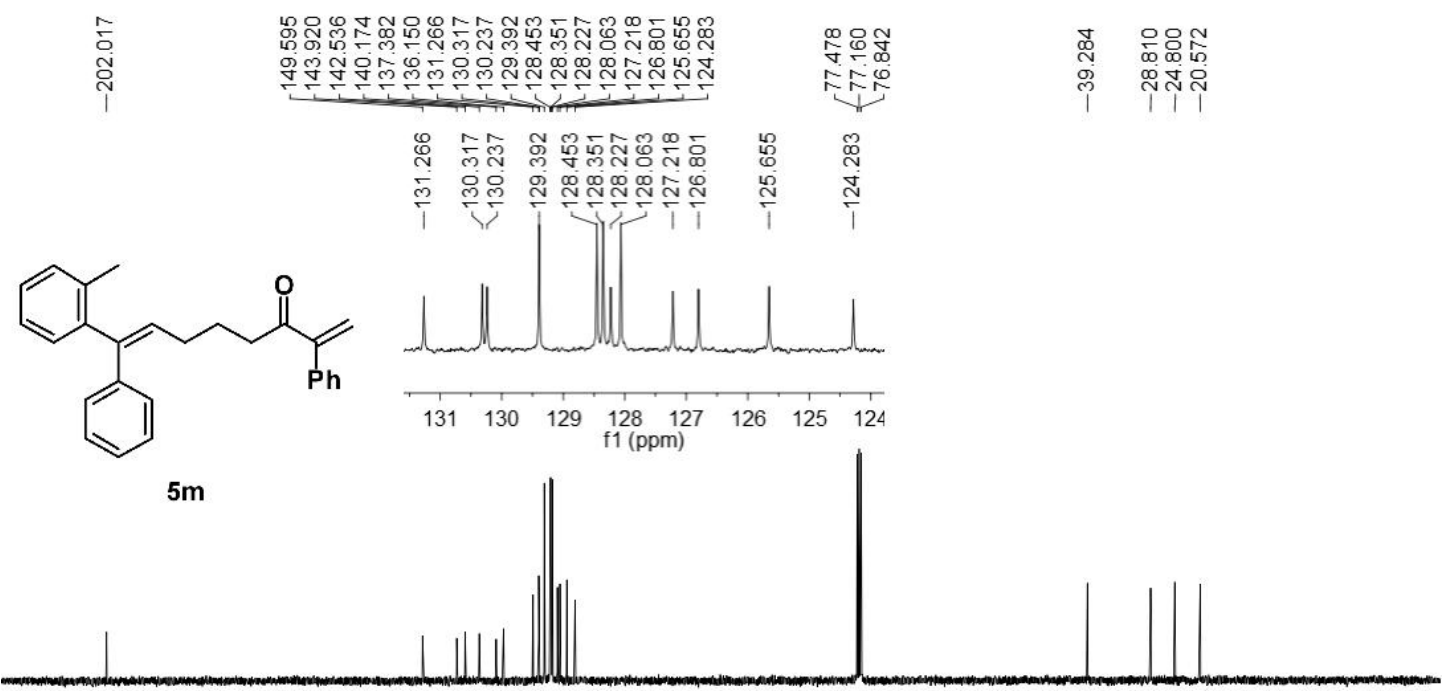

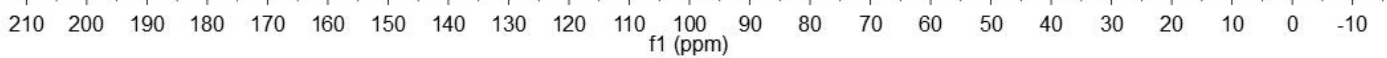


WQN-1760W

1H NMR in $\mathrm{CDCl} 3(400 \mathrm{MHz})$

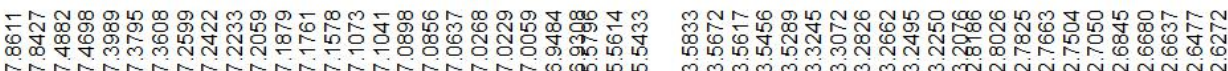

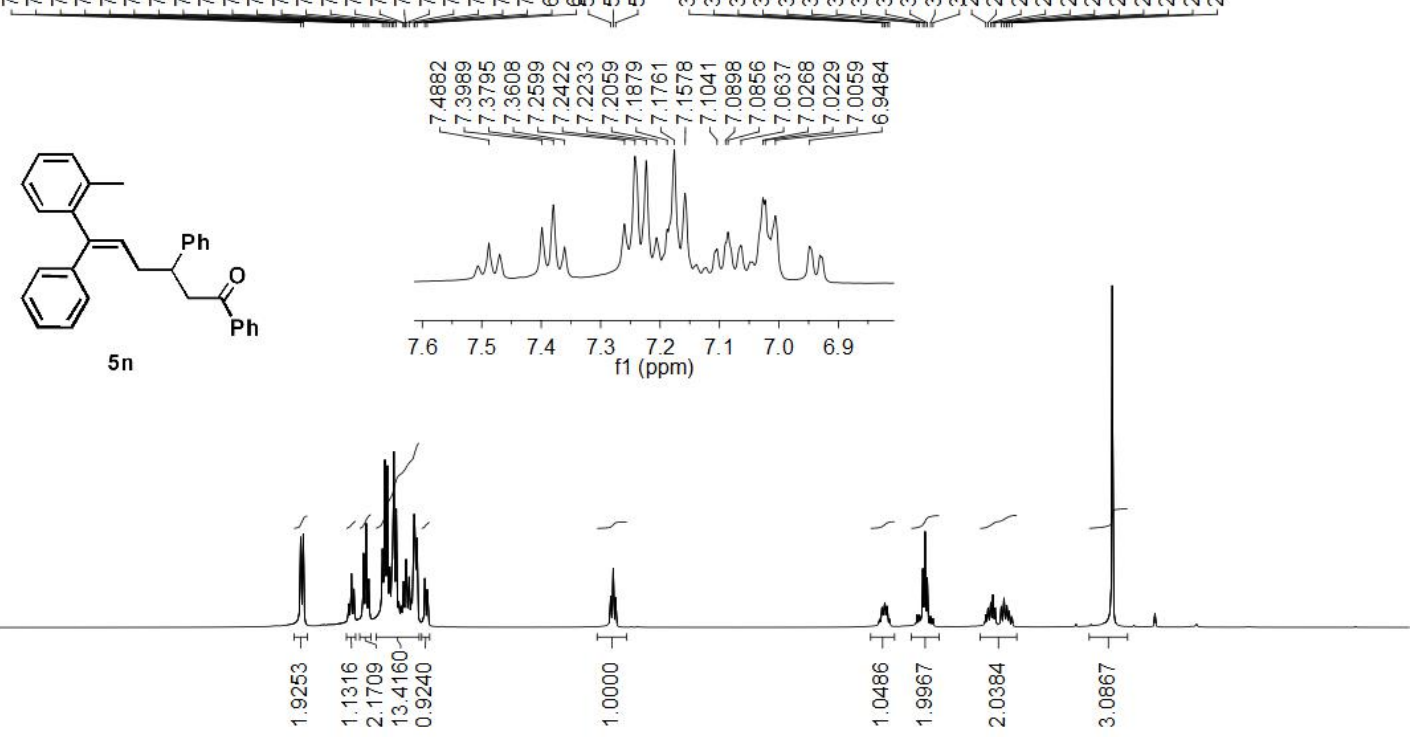



WQN-1760W

$13 \mathrm{C}$ NMR in $\mathrm{CDCl} 3(100 \mathrm{MHz})$

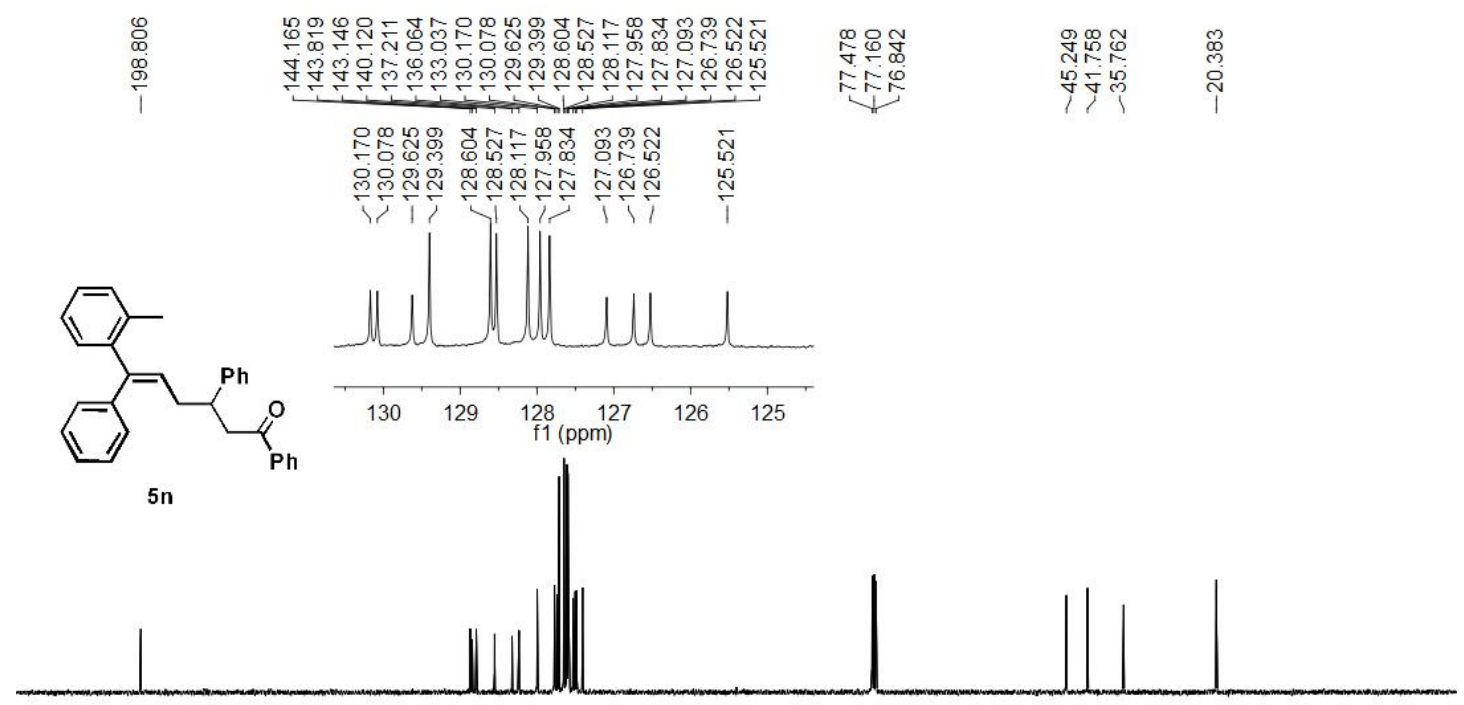

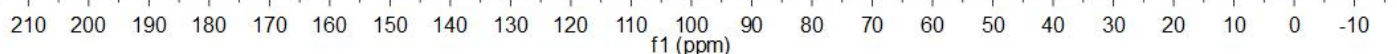




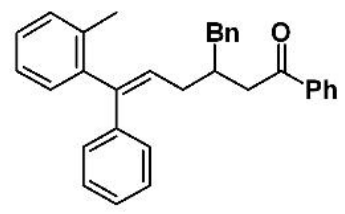

50

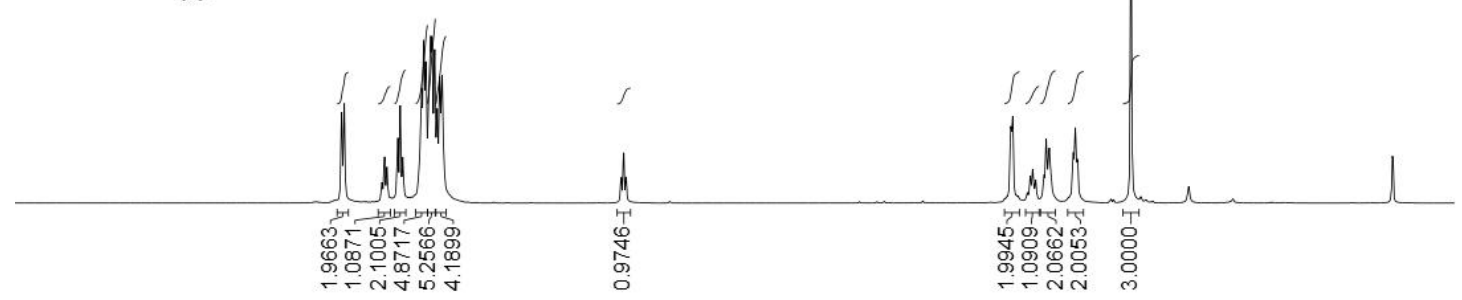

$\begin{array}{lllllllllllllllllllll}10.0 & 9.5 & 9.0 & 8.5 & 8.0 & 7.5 & 7.0 & 6.5 & 6.0 & 5.5 & \begin{array}{l}5.0 \\ \mathrm{f} 1(\mathrm{ppm})\end{array} & 4.5 & 4.0 & 3.5 & 3.0 & 2.5 & 2.0 & 1.5 & 1.0 & 0.5 & 0.0\end{array}$

Desktop

LJ-D471 13C NMR in CDCl3
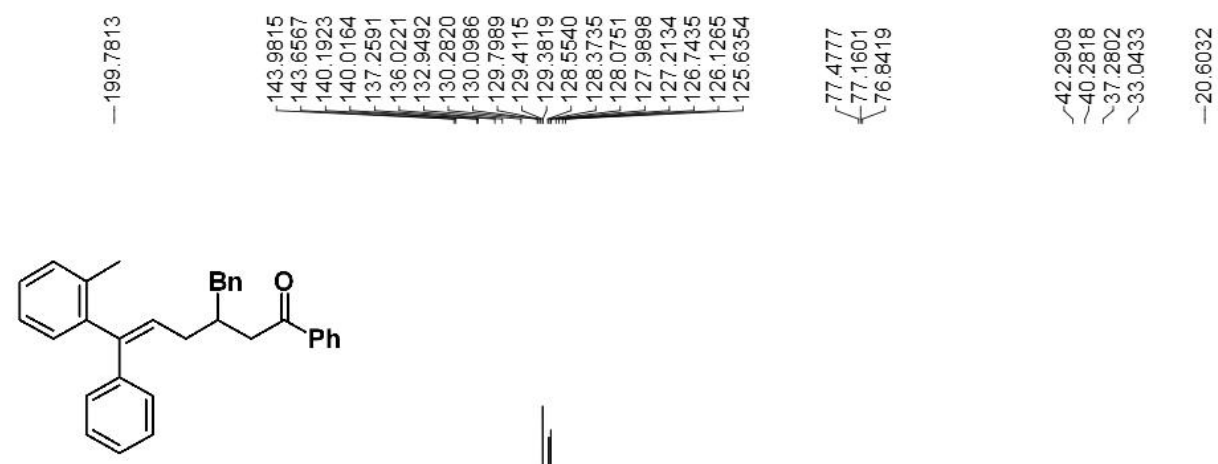

50

$\begin{array}{lllllllllllllllllllllll}210 & 200 & 190 & 180 & 170 & 160 & 150 & 140 & 130 & 120 & 110 & \begin{array}{c}100 \\ \mathrm{f} 1(\mathrm{ppm})\end{array} & 90 & 80 & 70 & 60 & 50 & 40 & 30 & 20 & 10 & 0 & -10\end{array}$ 

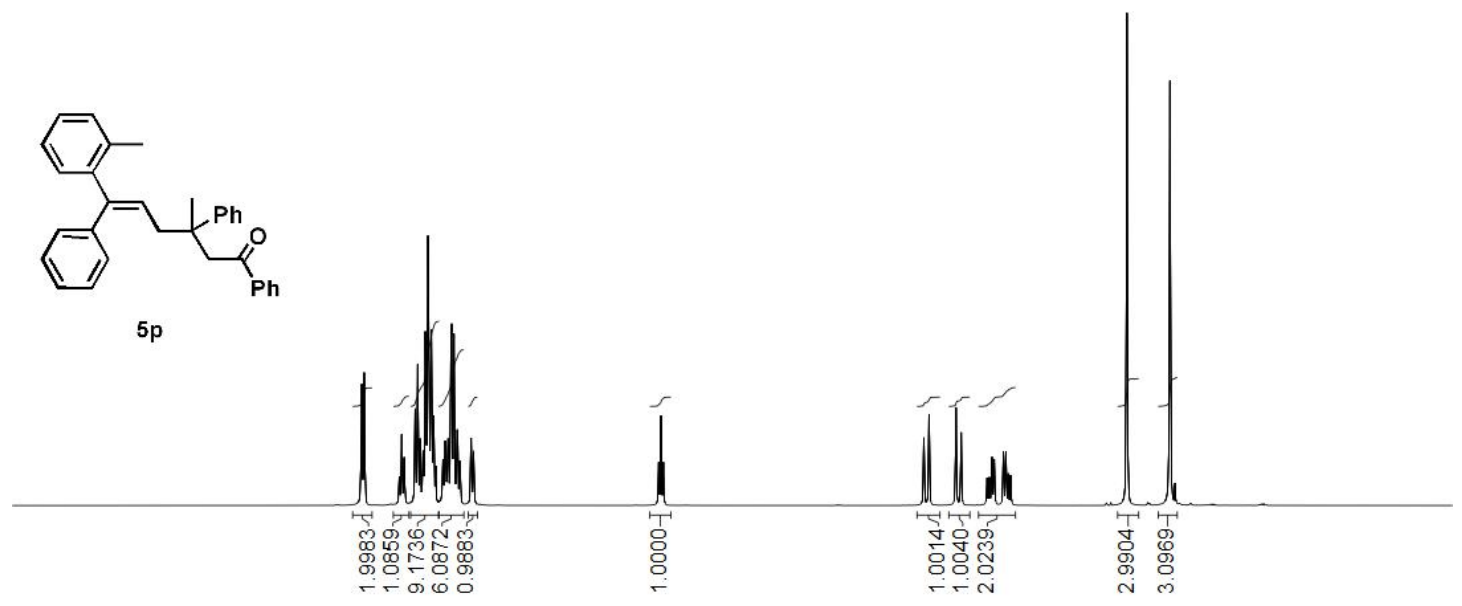

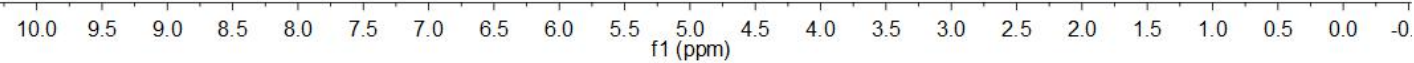

WQN-1751W

13C NMR in $\mathrm{CDCl} 3(100 \mathrm{MHz})$

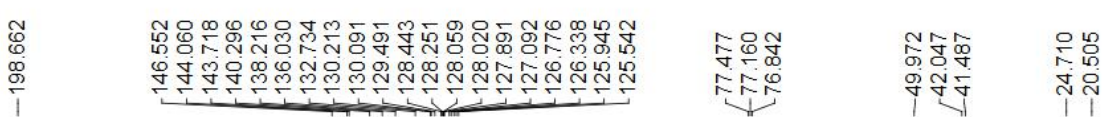

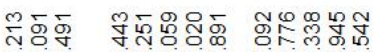

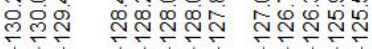

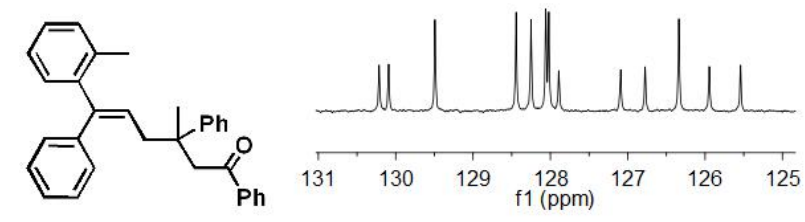

$5 p$

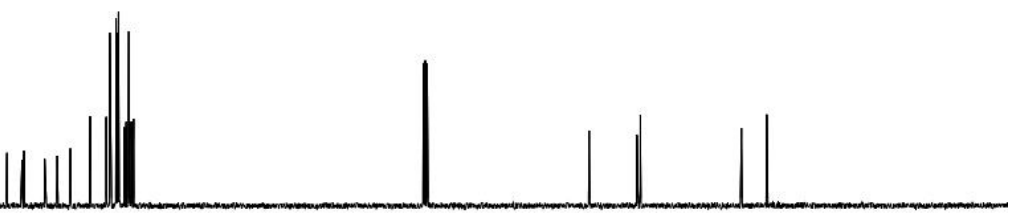

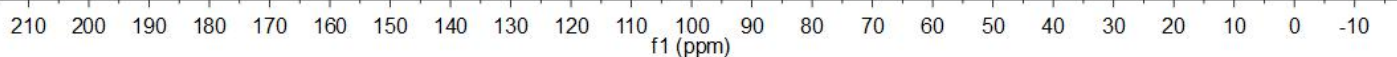


$1 \mathrm{H}$ NMR in $\mathrm{CDCl} 3(400 \mathrm{MHz})$

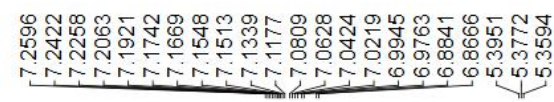

ब

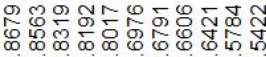

نัก

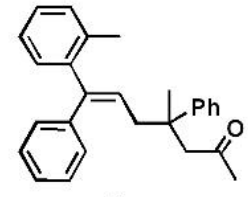

$5 q$
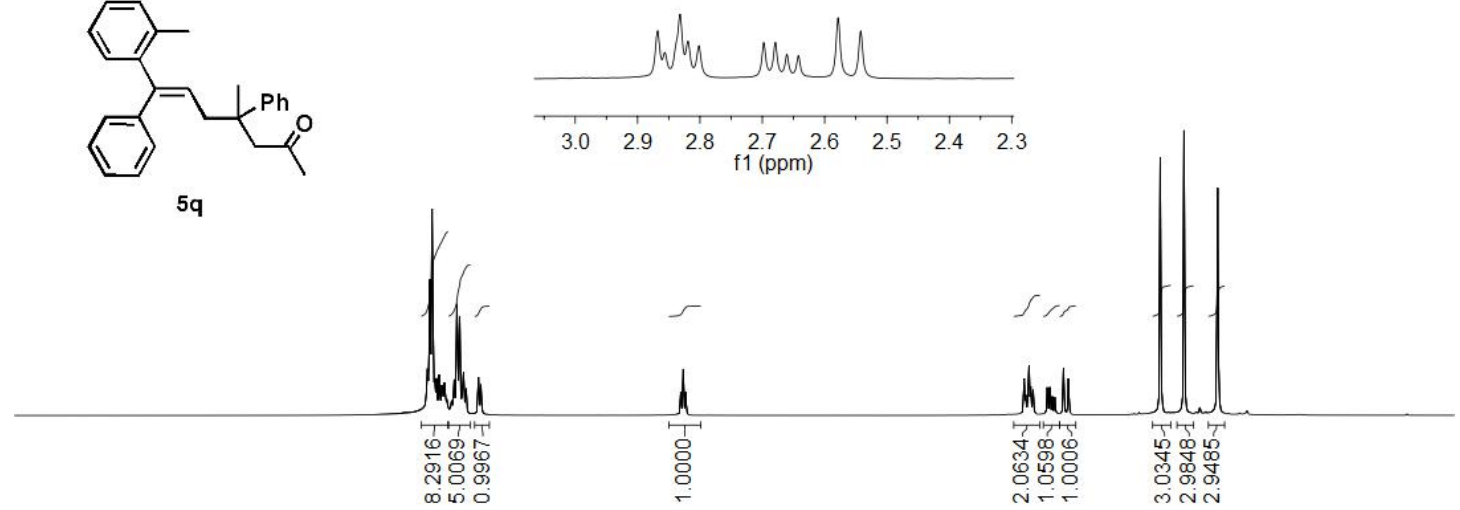

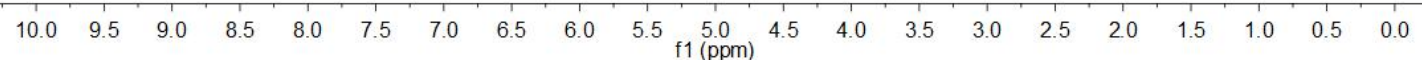

WQN-1756W

$13 \mathrm{C}$ NMR in $\mathrm{CDCl} 3(100 \mathrm{MHz})$

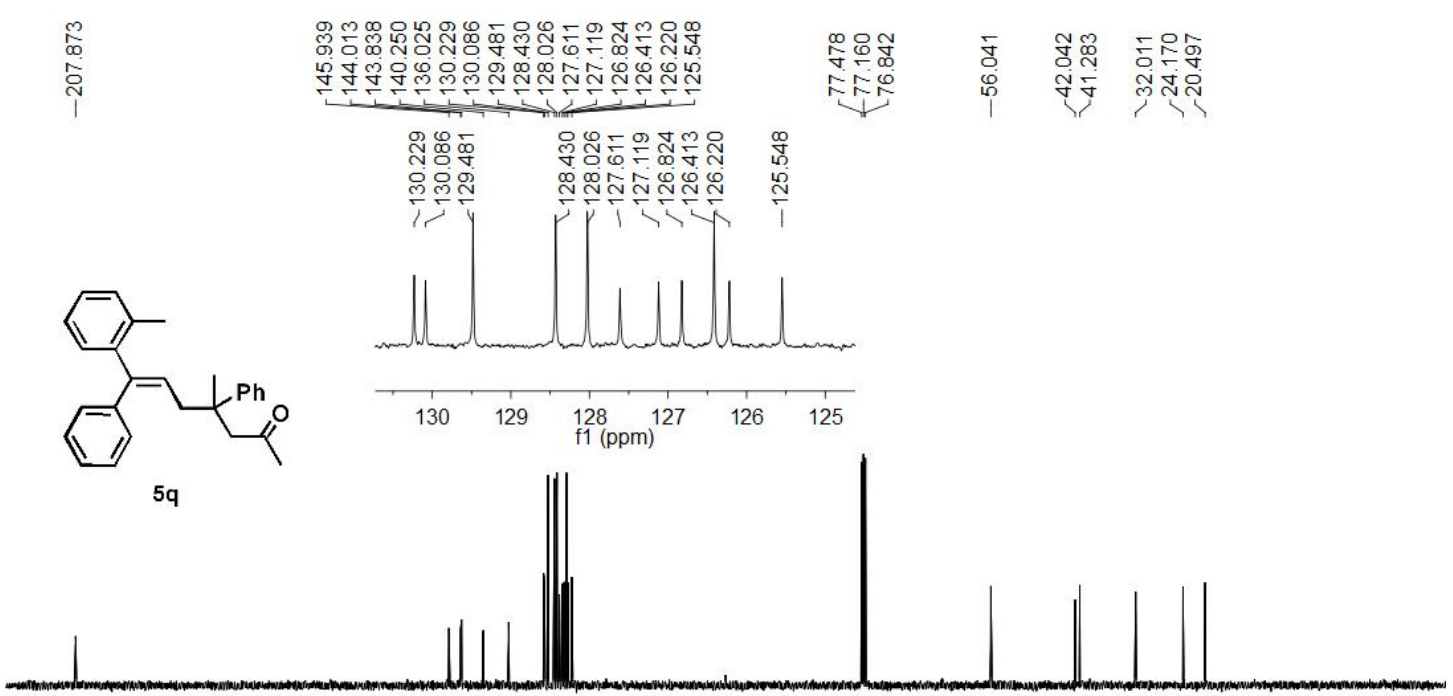

$\begin{array}{lllllllllllllllllllllll}210 & 200 & 190 & 180 & 170 & 160 & 150 & 140 & 130 & 120 & 110 & 100 & 90 & 80 & 70 & 60 & 50 & 40 & 30 & 20 & 10 & 0 & -10\end{array}$ 
WQN-1730W-1

PROTON CDC13 \{D: \NMR400\203\} nmr 25

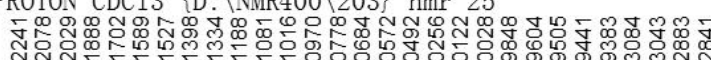

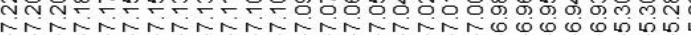

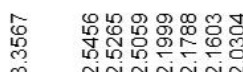

m NNNNNin

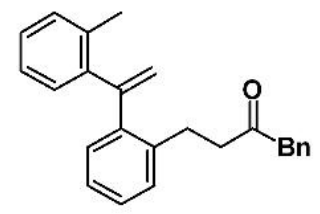

$8 a$

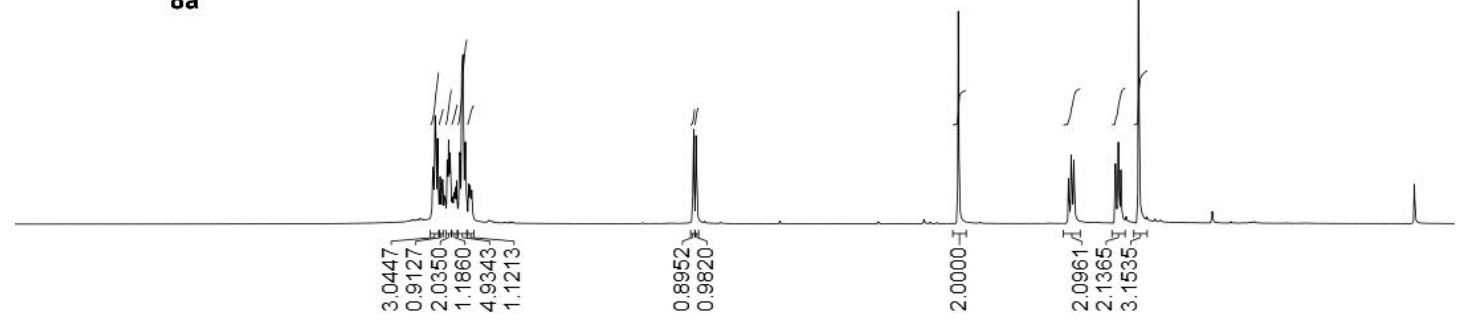

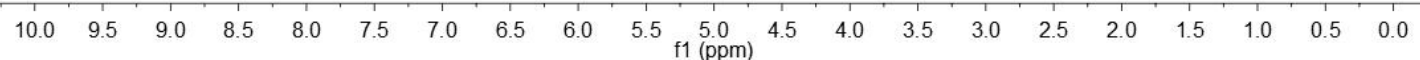

WQN-1730W-1

C13CPD CDC13 \{D: \NMR400\203\} $\mathrm{nmr} 25$

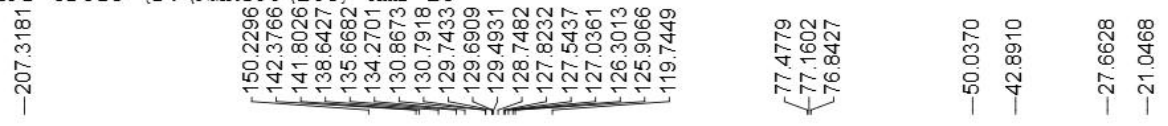

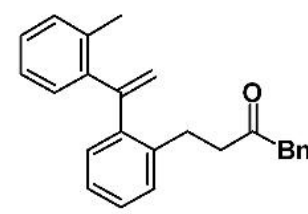

$8 \mathbf{a}$

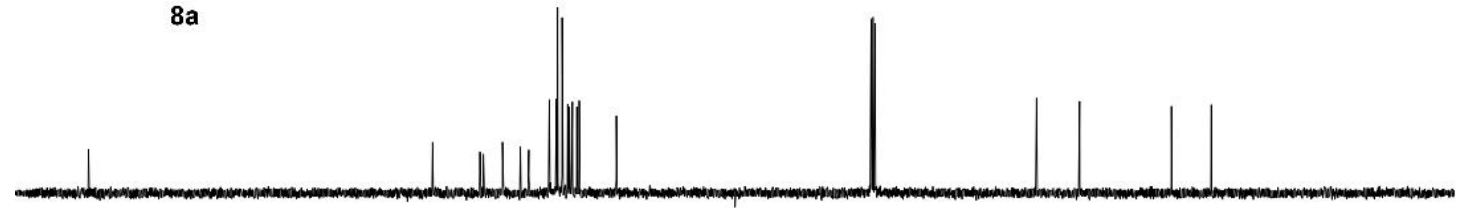

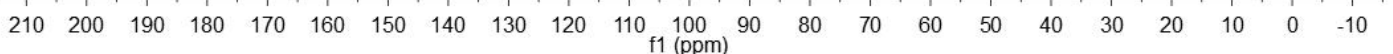






WQN-1755W

13C NMR in $\mathrm{CDCl} 3(100 \mathrm{MHz})$
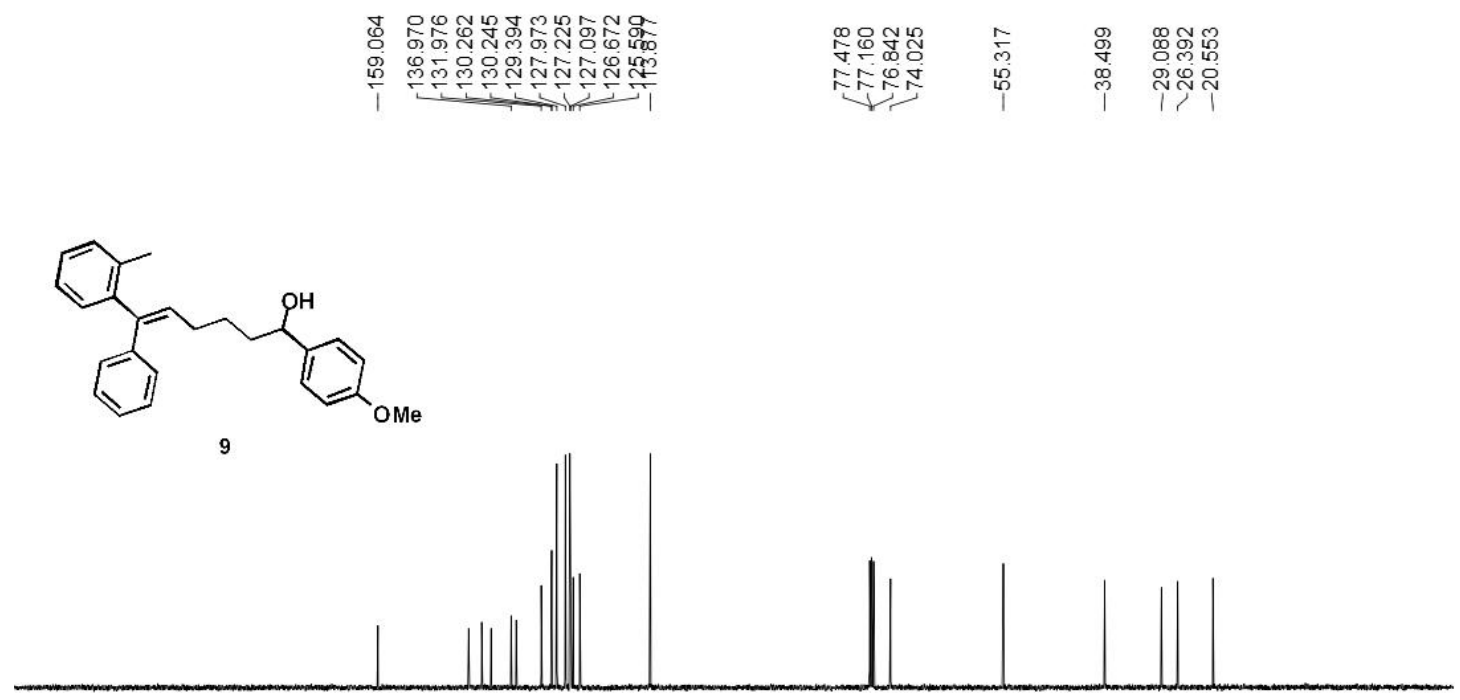

$\begin{array}{llllllllllllllllllllll}210 & 200 & 190 & 180 & 170 & 160 & 150 & 140 & 130 & 120 & 110 \begin{array}{c}100 \\ \mathrm{f} 1(\mathrm{ppm})\end{array} & 90 & 80 & 70 & 60 & 50 & 40 & 30 & 20 & 10 & 0 & -10\end{array}$ 
WQN-1750W

$1 \mathrm{H}$ NMR in $\mathrm{CDCl} 3(400 \mathrm{MHz})$
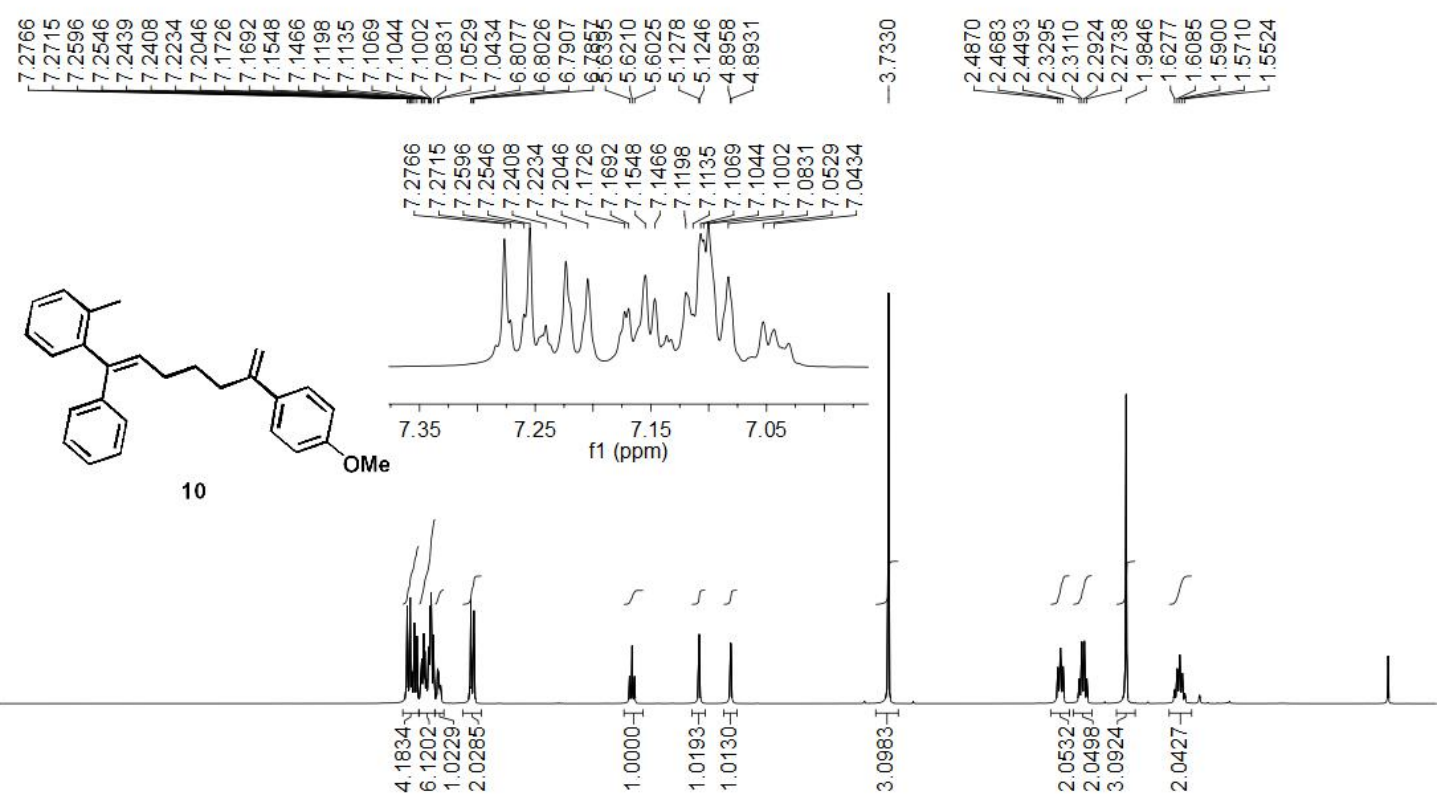

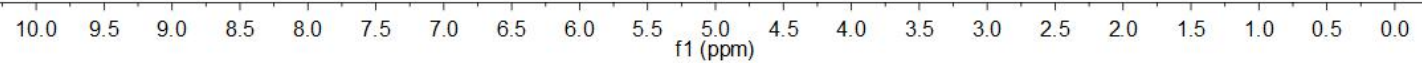

WQN-1750W

$13 \mathrm{C}$ NMR in $\mathrm{CDCl} 3(100 \mathrm{MHz})$

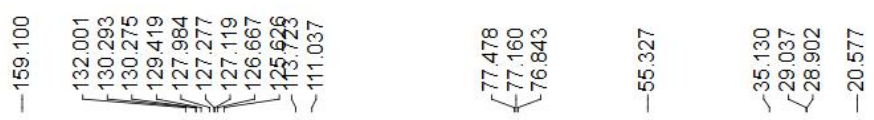

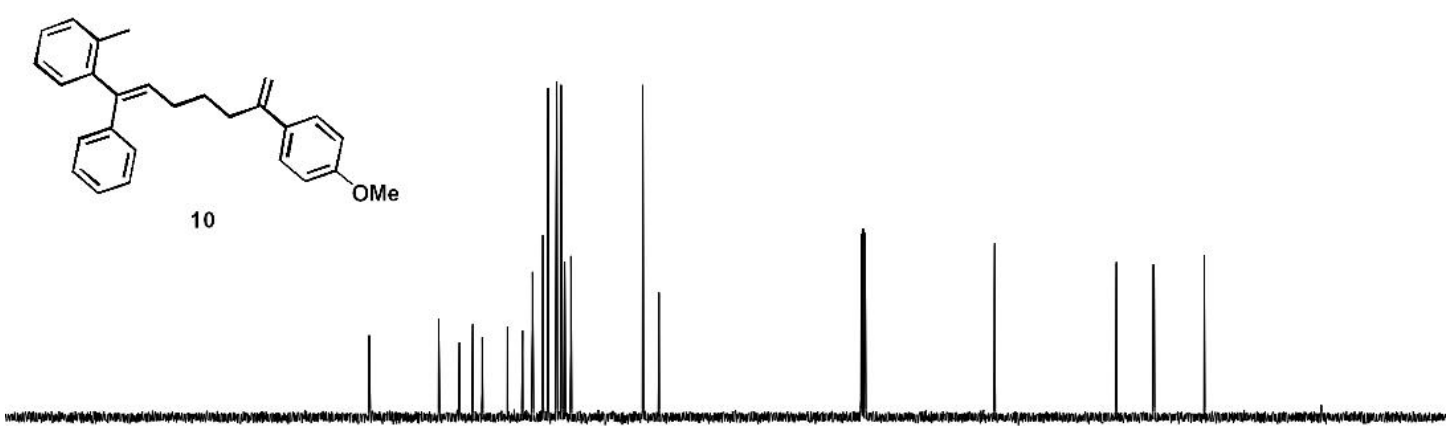

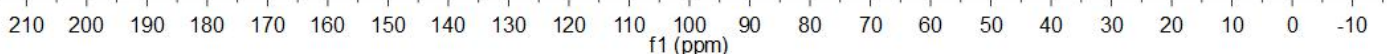

